Chemical Technology

Dunision

Chemica! mechnology

Dvision

Chemicall Technology

Division

Chemical Technology

Division

Chemical Technology

Division

Chemical Technology

Division

Chemical Technology

Division

Chemical Technology

Division

Chemica Technology

Divisum

Chemical Technology

Division

Ghemical Technology

Division

Chemical Technology

Division

Chemical Technology

Division

Chemical Technology

Division

Chemical Technology

Division

Chemical Technology

Division
RECFIVED

JAN 161995

OSTI

Characterization of Plutonium-Bearing Wastes by Chemical Analysis and Analytical Electron Microscopy

by R. G. Behrens, E. C. Buck, N. L. Dietz, J. K. Bates, E. Van Deventer, and D. J. Chaiko

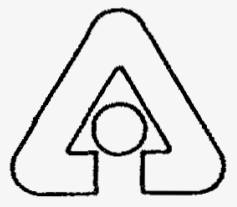

Argonne National Laboratory, Argonne, Illinois 60439

operated by The University of Chicago

for the United States Department of Energy under Contract W-31-109-Eng-38

Chemioal Technology

Duvision

Chemical Technology

Division

Chemical Technology

Division

Chemical Technology

Division 
Argonne National Laboratory, with facilities in the states of Illinois and Idaho, is owned by the United States government, and operated by The University of Chicago under the provisions of a contract with the Department of Energy.

\section{DISCLAIMER}

This report was prepared as an account of work sponsored by an agency of the United States Government. Neither the United States Government nor any agency thereof, nor any of their employees, makes any warranty, express or implied, or assumes any legal liability or responsibility for the accuracy, completeness, or usefulness of any information, apparatus, product, or process disclosed, or represents that its use would not infringe privately owned rights. Reference herein to any specific commercial product, process, or service by trade name, trademark, manufacturer, or otherwise, does not necessarily constitute or imply its endorsement, recommendation, or favoring by the United States Government or any agency thereof. The views and opinions of authors expressed herein do not necessarily state or reflect those of the United States Government or any agency thereof.

Reproduced from the best available copy.

Available to DOE and DOE contractors from the Office of Scientific and Technical Information

P.O. Box 62

Oak Ridge, TN 37831

Prices available from (423) 576-8401

Available to the public from the National Technical Information Service

U.S. Department of Commerce 5285 Port Royal Road Springfield, VA 22161 


\section{DISCLAMMER}

Portions of this document may be illegible in electronic image products. Images are produced from the best available original document. 
Distribution Category:

Nuclear Waste Management UC-510

ANL - 95/35

\section{CHARACTERIZATION OF PLUTONIUM-BEARING WASTES \\ BY CHEMICAL ANALYSIS AND \\ ANALYTICAL ELECTRON MICROSCOPY}

R. G. Behrens

Materials Science Technology Division

Los Alamos National Laboratory

Los Alamos, New Mexico 87545

and

E. C. Buck, N. L. Dietz, J. K. Bates,

E. Van Deventer, and D. J. Chaiko

Chemical Technology Division

Argonne National Laboratory

Argonne, Illinois 60439 



\section{CONTENTS}

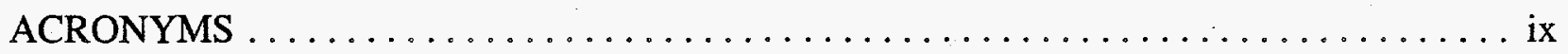

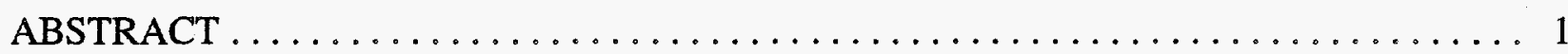

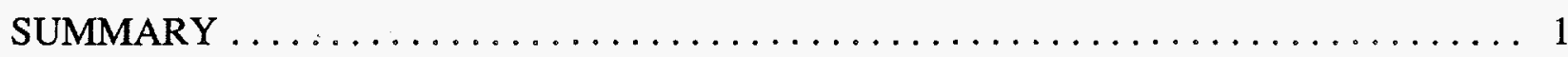

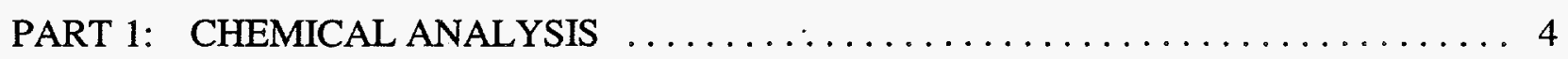

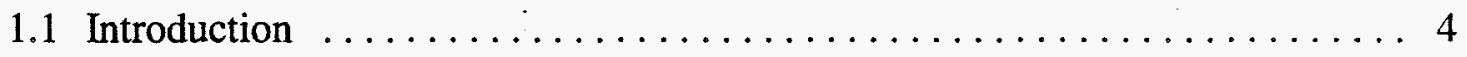

1.2 Incineration of Plutonium-Containing Combustible Materials

in the Defense Programs Complex ..................... 5

1.2.1 Los Alamos National Laboratory $\ldots \ldots \ldots \ldots \ldots \ldots \ldots \ldots \ldots$

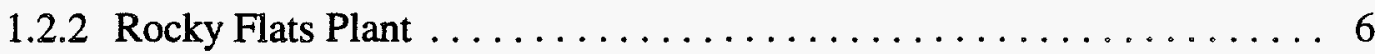

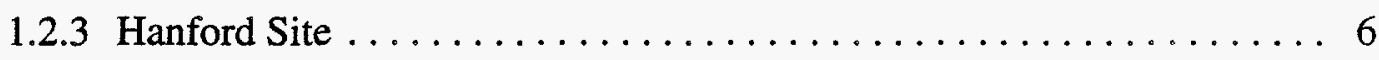

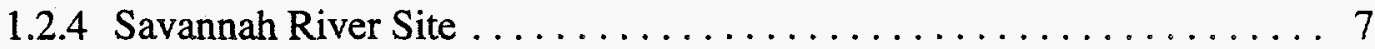

1.3 Incinerator Ash and Ash Heel Characterization $\ldots \ldots \ldots \ldots \ldots \ldots \ldots \ldots$

1.3.1 Literature Review . . . . . . . . . . . . . . . . . . . . 7

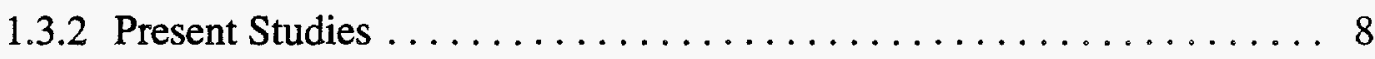

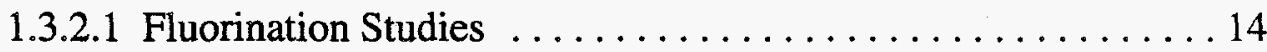

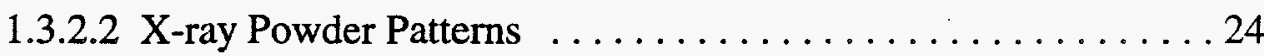

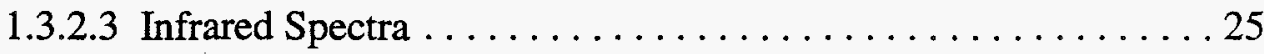

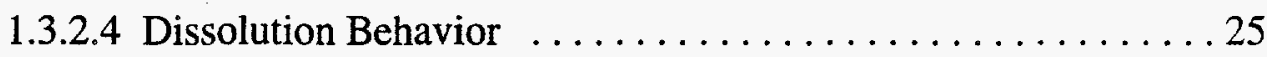

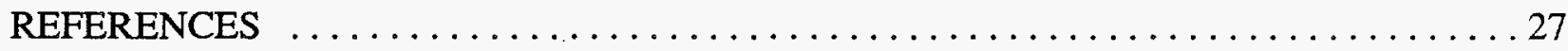

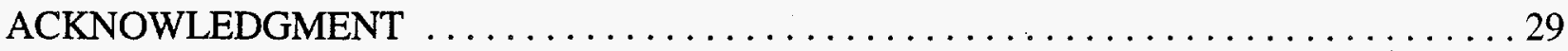

PART 2: ANALYTICAL ELECTRON MICROSCOPY

CHARACTERIZATION $\ldots \ldots \ldots \ldots \ldots \ldots \ldots \ldots \ldots \ldots \ldots \ldots \ldots \ldots \ldots \ldots \ldots \ldots$

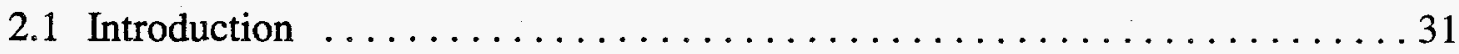

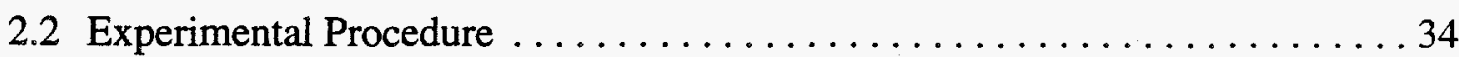

2.2 .1 Microscopic Analysis . . . . . . . . . . . . . . . . . . . 34

2.2.2 Preparation of Samples for Scanning Electron Microscopy . . . . . . 34

2.2.3 Analysis of Samples by Analytical Electron Microscopy .......... 34

2.2.4 Particle Size Reduction ............................. 37 
CONTENTS (Cont.)

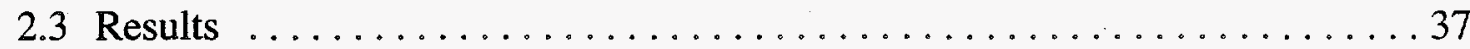

2.3.1 Plutonium Distribution in the Waste Particle Matrix $\ldots \ldots \ldots . . . .37$

2.3.2 Survey of Samples .............................. 39

2.3.3 Detailed Analysis of Samples . . . . . . . . . . . . . . . . . 39

2.3.3.1 Los Alamos Ash ............................ 39

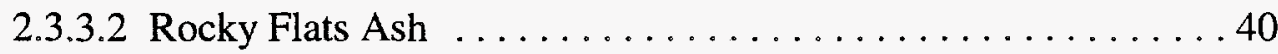

2.3.3.3 Rocky Flats Ash Heel ....................... 42

2.3.3.4 Sand, Slag, and Crucible Samples $\ldots \ldots \ldots \ldots \ldots \ldots \ldots 6 . \ldots \ldots$

2.3.3.5 Ground LECO Crucible ..................... . 70

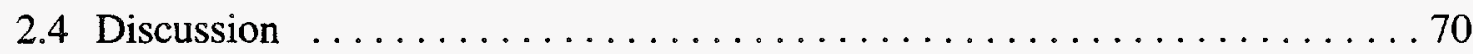

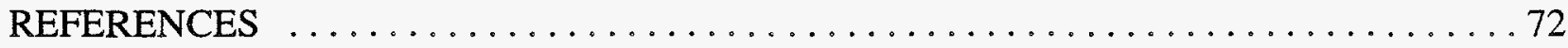

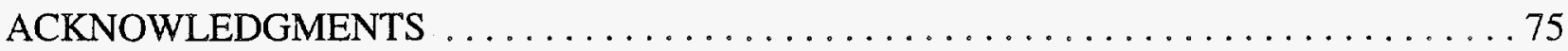

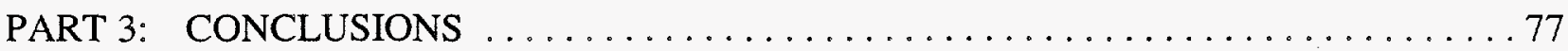

\section{TABLES}

1.1 Chemical Composition of Typical Rocky Flats Incinerator Ash and Ash Heel $\ldots \ldots 8$

1.2 Chemical Composition of Rocky Flats Incinerator Ash by Age $\ldots \ldots \ldots \ldots \ldots$

1.3 Chemical Composition of Rocky Flats Incinerator Ash Heel by Age and Ash-to-Leachate Ratio

1.4 Comparison of Plutonium Content of Virgin Los Alamos Incinerator Ash as Measured by Several Techniques

1.5 Elemental Analyses for Three Batches of Virgin

Los Alamos Incinerator Ash

1.6 Elemental Analyses for Three Batches of Virgin Rocky Flats

Incinerator Ash 


\section{TABLES (Cont.)}

1.7 Elemental Analyses for Two Batches of Calcined Rocky Flats

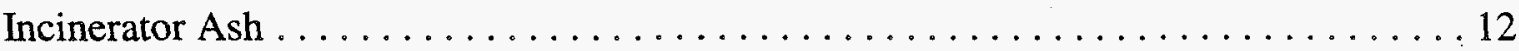

1.8 Summary of Loss-On-Ignition Data for Incinerator Ash Samples . . . . . . . . . . . 13

$1.9 \quad$ Summary of Sieving Studies on Incinerator Ash Samples $\ldots \ldots \ldots \ldots \ldots \ldots$

1.10. Elemental Composition of Filter Residue and Dissolver Heel Obtained during Small-Scale Cascade Dissolution Studies of Calcined Rocky Flats

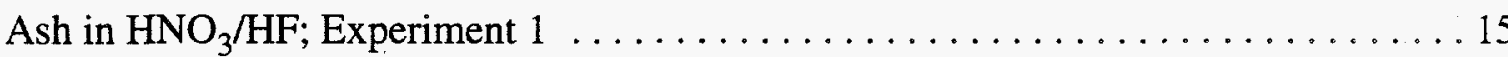

1.11 Elemental Composition of Filter Residue and Dissolver Heel Obtained during Small-Scale Cascade Dissolution Studies of Calcined Rocky Flats

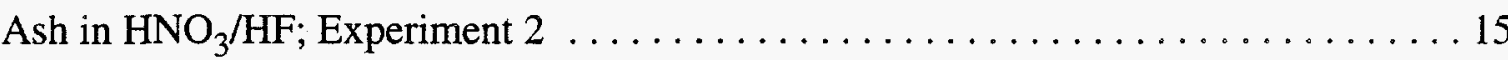

1.12 Elemental Composition of Filter Residue and Dissolver Heel Obtained during Small-Scale Cascade Dissolution Studies of Calcined Rocky Flats

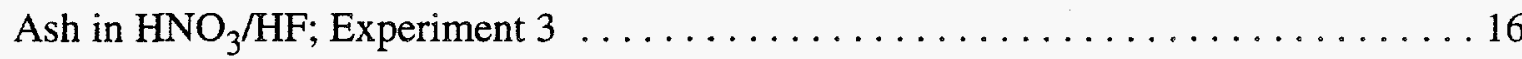

1.13 Elemental Composition of Filter Residue and Dissolver Heel Obtained during Small-Scale Cascade Dissolution Studies of Calcined Rocky Flats

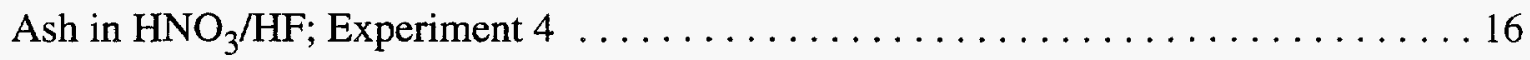

1.14 Elemental Composition of Filter Residue and Dissolver Heel Obtained during Small-Scale Cascade Dissolution Studies of Calcined Rocky Flats Ash in $\mathrm{HNO}_{3} / \mathrm{HF}$; Experiment 5

1.15 Elemental Composition of Filter Residue and Dissolver Heel Obtained during Small-Scale Cascade Dissolution Studies of Calcined Rocky Flats Ash in $\mathrm{HNO}_{3} / \mathrm{HF}$; Experiment 6

1.16 Elemental Composition of Los Alamos Incinerator Ash after Fluorination at

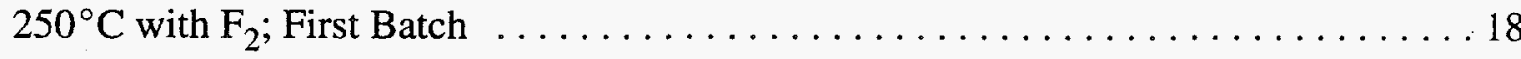

1.17 Elemental Composition of Los Alamos Incinerator Ash after Fluorination at $250^{\circ} \mathrm{C}$ with $\mathrm{F}_{2}$; Second Batch 


\section{TABLES (Cont.)}

1.18 Elemental Composition of Los Alamos Incinerator Ash after Fluorination at $500^{\circ} \mathrm{C}$ with $\mathrm{F}_{2}$

1.19 Elemental Composition of Filter Residue and Dissolver Heel Obtained during Small-Scale Cascade Dissolution Studies of Prefluorinated Virgin Los Alamos Ash in $\mathrm{HNO}_{3}$

1.20 Summary of X-ray Diffraction Results on Virgin and Fluorinated Los Alamos Ash

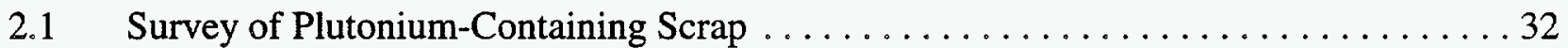

2.2 Summary of Results from Sample Surveys $\ldots \ldots \ldots \ldots \ldots \ldots \ldots \ldots$

2.3 Electron Diffraction Analysis from Plutonium Oxide Particles in Los Alamos Ash . . . . . . . . . . . . . . . . . . . . . . . . . . . . . . . . . . . 48

2.4 Electron Diffraction from Plutonium Oxide Particles in Rocky Flats Ash Heel . . . . . 59

2.5 Electron Diffraction from Crystalline Plutonium Phases in Rocky Flats Ash Heel

2.6 Electron Diffraction from Non-Plutonium-Bearing Phases

in Rocky Flats Ash Heel

2.7 Quantitative Analysis of Plutonium-Bearing Glassy Phases in Rocky Flats Ash Heel

2.8 Electron Diffraction from Plutonium Oxide in LECO Crucible 64 


\section{FIGURES}

1.1 FTIR Spectrum Showing Absorbances for Major Vapor Species Produced during Fluorination of Los Alamos Incinerator Ash at $250^{\circ} \mathrm{C}$ Using $\mathrm{F}_{2} \ldots \ldots \ldots 20$

1.2 FTIR Spectrum Showing the Brief Presence of the Superfluorinating

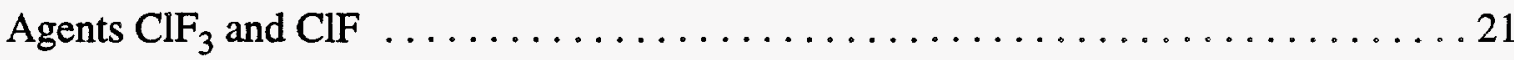

1.3 FTIR Spectrum Obtained 20 min after Spectrum Shown in Fig. $1.2 \ldots \ldots \ldots \ldots .21$

1.4 FTIR Spectrum Taken at the Beginning of Fluorination of Los Alamos Ash

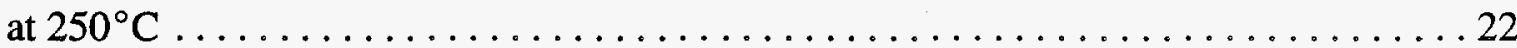

1.5 FTIR Spectrum Taken at the End of Fluorination of Los Alamos Ash

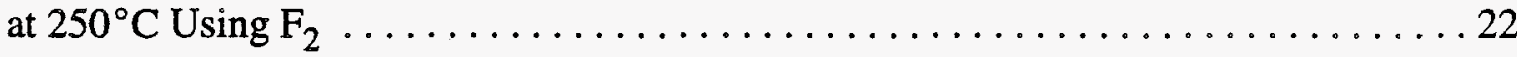

1.6 Infrared Spectra of (a) Virgin Ash, (b) Fluorinated Ash, and (c) $\mathrm{K}_{\mathrm{x}} \mathrm{UO}_{2} \mathrm{~F}_{2+\mathrm{x}} \ldots \ldots 26$

2.1 Analytical Electron Microscopy Facility at Argonne National Laboratory . . . . . . . . 36

2.2 Photomicrograph of an Ash Heel Particle Showing Plutonium Grains in a Matrix of Metal Oxides and $\mathrm{SiO}_{2} \ldots \ldots \ldots \ldots \ldots \ldots \ldots \ldots \ldots \ldots \ldots \ldots \ldots$

2.3 Energy Dispersive X-ray Spectrum of the Particle Matrix in Fig. $2.2 \ldots \ldots \ldots \ldots 38$

2.4 Energy Dispersive X-ray Spectrum of a $10-\mu \mathrm{m}$ Particle

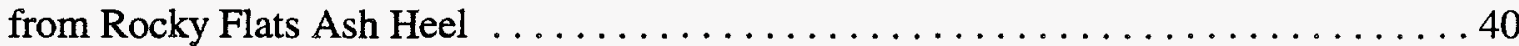

2.5 BSE Micrograph of -200 Mesh Fraction from a Crushed LECO Crucible

2.6 BSE Micrograph of +200 Mesh Fraction from a Crushed LECO Crucible

2.7 BSE Image of Plutonium-Bearing Particles from a Crushed LECO Crucible

2.8 Low Magnification Micrograph of Thin Section of Plutonium and Other Particles 


\section{FIGURES (Cont.)}

2.9 Typical Energy Dispersive X-ray Spectrum of a -200 Mesh

Particle from a Crushed LECO Crucible . . . . . . . . . . . . . . . . . 44

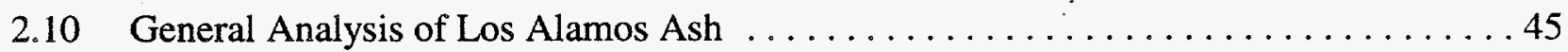

2.11 Analysis of Plutonium Oxide Particles in Los Alamos Ash . . . . . . . . . . 46

2.12 Analysis of Plutonium Oxide Particles in Rocky Flats Ash . . . . . . . . . . . . 49

2.13 Further Analysis of Particles in Rocky Flats Ash $\ldots \ldots \ldots \ldots \ldots \ldots \ldots \ldots$

2.14 (a) Partial Kikuchi Map of Plutonium Oxide in Rocky Flats Ash and (b) EELS Analysis of the $\mathrm{Pu}^{-\mathrm{O}_{4,5}}$ Edges $\ldots \ldots \ldots \ldots \ldots \ldots \ldots \ldots \ldots \ldots$

2.15 Analyses of Plutonium-Bearing and Non-Plutonium-Bearing Particles

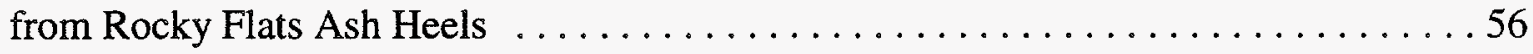

2.16 Analyses of Plutonium Titanium Silicate Phase from Rocky Flats Ash Heel . . . . . 60

2.17 (a) TEM Image of a Plutonium-Bearing Silicate Glass from Rocky Flats Ash Heel and (b) EDS Analysis of Glassy Phase

2.18 EDS Analysis of Alumino-Plutonium Phase from Rocky Flats Ash Heel . . . . . 64

2.19 Analyses of Plutonium Oxide and Other Particles from Sand, Slag, and Crucible Samples

2.20 TEM Images of Plutonium Oxide and Other Particles from Ground LECO Crucible Sample

2.21 EDS Analyses of (a) Plutonium Oxide Phase, (b) Plutonium Silicotitanate Phase, and (c) Non-Plutonium-Bearing Particles Containing Calcium Aluminosilicate in Ground LECO Crucible Sample 


\section{ACRONYMS}

AEM analytical electron microscopy

BSE backscattering electron

CCD charge-coupled device

DP Defense Programs

DOE U.S. Department of Energy

EDL economic discard limit

EDS energy dispersive spectrometry

EELS electron energy loss spectrometry

FTIR Fourier transform infrared [spectrometry]

IDMS isotope dilution mass spectrometry

LOI weight-loss-on-ignition

SAI solution assay instrument

SEM scanning electron microscopy

TEM transmission electron microscopy

XRD X-ray diffraction 


\title{
CHARACTERIZATION OF PLUTONIUM-BEARING WASTES BY CHEMICAL ANALYSIS AND ANALYTICAL ELECTRON MICROSCOPY
}

\begin{abstract}
This report summarizes the results of characterization studies of plutoniumbearing wastes produced at the U.S. Department of Energy weapons production facilities. Several different solid wastes were characterized, including incinerator ash and ash heels from Rocky Flats Plant and Los Alamos National Laboratory; sand, slag, and crucible waste from Hanford; and LECO crucibles from the Savannah River Site. These materials were characterized by chemical analysis and analytical electron microscopy. The results showed the presence of discrete $\mathrm{PuO}_{2}$, $\mathrm{PuO}_{2}-\mathrm{x}$, and $\mathrm{Pu}_{4} \mathrm{O}_{7}$ phases, of about $1 \mu \mathrm{m}$ or less in size, in all of the samples examined. In addition, a number of amorphous phases were present that contained plutonium. In all the ash and ash heel samples examined, plutonium phases were found that were completely surrounded by silicate matrices. Consequently, to achieve optimum plutonium recovery in any chemical extraction process, extraction would have to be coupled with ultrafine grinding to average particle sizes of less than $1 \mu \mathrm{m}$ to liberate the plutonium from the surrounding inert matrix.
\end{abstract}

\section{SUMMARY}

This report presents the results of a comprehensive study of the physicochemical characteristics of various plutonium-containing wastes. It is presented in two parts. The first summarizes the chemical characterization of plutonium-containing wastes produced at U.S. Department of Energy weapons production facilities. This work was carried out at Los Alamos National Laboratory. The second part of this report summarizes the analytical electron microscopy (AEM) analysis of these wastes that was conducted at Argonne National Laboratory. Several different plutonium-containing solid wastes were characterized, including incinerator ash and ash heels from Rocky Flats Plant and Los Alamos National Laboratory; sand, slag, and crucible waste from Hanford; and LECO crucibles from the Savannah River Site.

In the first part of this report, we present a comprehensive study of incinerator ash and ash heel chemistry. We review the incineration processes that were used in treating combustible waste materials produced in the defense programs complex. A literature survey is presented that details what is known about the chemical composition of plutonium-containing incinerator ash. The focus 
of this study was to provide a more detailed chemical analysis of the incinerator ash and ash heels produced at Los Alamos National Laboratory and the incinerator ash produced at the Rocky Flats Plant. The various plutonium-bearing samples were characterized by weight-loss-on-ignition studies, size fractionation, X-ray diffraction, elemental analysis, infrared spectroscopy, fluoride volatility analysis (using Fourier transform infrared spectroscopy as a diagnostic), and electron microprobe analysis.

Chemical analysis of Los Alamos ash samples showed that the major components (those present at $1 \mathrm{wt} \%$ or greater) were $\mathrm{Al}, \mathrm{C}, \mathrm{Ca}, \mathrm{Cl}, \mathrm{Cr}, \mathrm{Fe}, \mathrm{K}, \mathrm{Mg}, \mathrm{Mn}, \mathrm{Mo}, \mathrm{Na}, \mathrm{Ni}, \mathrm{Pu}, \mathrm{Si}$, and $\mathrm{Zn}$. The only major difference between the Rocky Flats and Los Alamos ashes was that $\mathrm{Ba}, \mathrm{Pb}$, and $\mathrm{Ti}$ were observed in the Rocky Flats ash samples.

Results from sieving experiments indicate that no major fractionation of plutonium occurs during simple size classification. Thus, concentrating plutonium in a small-particle-size fraction of the ash does not appear feasible without prior physical treatment such as grinding.

Infrared spectra taken during fluorination experiments showed evidence of the presence of $\mathrm{ClF}_{3}$ and $\mathrm{CIF}$ at temperatures of $250^{\circ} \mathrm{C}$, but while $\mathrm{C}, \mathrm{Si}, \mathrm{S}$, and $\mathrm{P}$ were volatilized during fluorination, there was no evidence that the conversion of $\mathrm{Pu}$ to $\mathrm{PuF}_{6}(\mathrm{~g})$ occurred.

The AEM characterization of various plutonium-containing wastes included optical microscopy and various electron microscopies that together provide detailed information about particle morphology and the juxtaposition of various phases within individual particles. Various probes, such as X-ray energy dispersive spectrometers (EDSs) and a Gatan parallel electron energy loss spectrometer (EELS), were used with electron diffraction to identify the various phases present in individual particles. We also examined the pattern of particle breakage during ultrafine grinding of the samples. This analysis revealed evidence of nonuniform breakage, but the degree of plutonium liberation was highly variable from one waste material to another. The highest degree of plutonium liberation was observed in ground LECO crucibles, which showed two discrete populations of particles - those containing large amounts of plutonium and those with nondetectable levels of plutonium.

$\mathrm{X}$-ray powder diffraction measurements of ash and ash heels indicated the presence of $\mathrm{PuO}_{2}$ phases with moderate crystallinity. In addition, electron diffraction analysis and EDS analyses showed the presence of individual $\mathrm{PuO}_{2}, \mathrm{PuO}_{2-\mathrm{x}}$, and $\mathrm{Pu}_{4} \mathrm{O}_{7}$ phases in these materials. There were also a number of amorphous phases present that contained plutonium. Electron microscopy showed the presence of discrete plutonium phases, of about $1 \mu \mathrm{m}$ or less in size, in all of the samples examined. The most dramatic images were obtained from crushed LECO crucibles; these images showed that all detectable plutonium was present as discrete plutonium phases of about $1 \mu \mathrm{m}$ in diameter located within the grain boundaries of a copper-containing matrix. 
In all the ash and ash heel samples examined, plutonium phases were found that were completely surrounded by silicate matrices. Consequently, to achieve optimum plutonium recovery in any selective chemical extraction process, extraction would have to be coupled with ultrafine grinding to average particle sizes of less than $1 \mu \mathrm{m}$ to liberate the plutonium from the surrounding inert matrix.

Although the most abundant plutonium-bearing phase found in all the waste samples was a reduced plutonium oxide (e.g., $\mathrm{Pu}_{4} \mathrm{O}_{7}$ ), there was evidence of other plutonium-bearing phases. In the Rocky Flats ash samples, for example, plutonium-bearing aluminosilicates, plutonium silicotitanate phases, an aluminum-plutonium phase, and glassy aluminosilicate phases containing approximately $10 \mathrm{wt} \%$ plutonium were observed. The presence of the glassy plutonium phase and other plutonium silicates suggests that the incinerator may have operated at temperatures much higher than previously believed (i.e., $>900^{\circ} \mathrm{C}$ ), as these types of phases are unlikely to form at temperatures between $200-900^{\circ} \mathrm{C}$.

In general, characterization of the plutonium incinerator wastes proved difficult because the database of plutonium phases, other than simple oxides, is extremely limited. Further analysis will be required to determine exactly the identity of some of the plutonium-bearing phases found in this study. 


\title{
PART 1: CHEMICAL ANALYSIS
}

by

\author{
R.G. Behrens
}

\subsection{INTRODUCTION}

It has been estimated that approximately $36,000 \mathrm{~kg}$ (bulk weight) of plutonium-containing residues are in storage at the Rocky Flats Plant. This material is estimated to contain approximately $1,300 \mathrm{~kg}$ of plutonium. The $36,000 \mathrm{~kg}$ of bulk residue material comprises approximately $18,000 \mathrm{~kg}$ of incinerator ash plus incinerator ash heel. (Ash heel is the solid residue remaining after $\mathrm{HNO}_{3} / \mathrm{HF}$ dissolution of incinerator ash to extract the plutonium.) The ash and ash heel in storage at Rocky Flats contain approximately $900 \mathrm{~kg}$ of plutonium metal. Incinerator ash and ash heel thus contain approximately $70 \%$ of all the plutonium present in waste residues in storage at Rocky Flats. Add to this the plutonium present in ash or in combustible wastes in storage at Los Alamos National Laboratory, the Hanford Site, the Savannah River Site, and Lawrence Livermore National Laboratory, and one can readily understand the recent increase in interest in effectively retrieving plutonium from these waste residues.

Considerable effort has gone into understanding the leaching mechanism of plutonium from incinerator ash and ash heel during $\mathrm{HNO}_{3} / \mathrm{HF}$ dissolution [KAZANJIAN, TALLENT, BARNEY, RYAN]. However, little effort has gone into understanding the basic chemistry and detailed chemical composition of both ash and ash heel.

Incinerator ash is considered to be a lean residue material with plutonium content on the order of 5 to $15 \mathrm{wt} \%$. Ash is also a refractory material because the plutonium is present as a highfired oxide. While it is known that ash forms in incinerators somewhere between $200^{\circ} \mathrm{C}$ and $900^{\circ} \mathrm{C}$ (the combustion temperature is strongly dependent on the material being burned [CHRISTENSEN1988]), it is not known, for example, what the chemical form of plutonium is within the ash. Is the plutonium present as discrete oxide particles or agglomerates physically attached to other particles? Is the plutonium chemically alloyed with other chemical constituents to form refractory ternary oxides or more complex chemical species that are even more thermodynamically stable than $\mathrm{PuO}_{2}$ and thus resist dissolution even more? How varied is the chemical composition of the ash presently in storage? How, for example, does the chemistry of ash produced at Rocky Flats differ from ash produced at Los Alamos, and how does this chemical difference affect dissolution chemistry?

Part 1 of this report is an attempt to answer some of the above questions concerning the chemistry of plutonium-bearing incinerator ash. Section 1.2 briefly summarizes incineration of combustible materials within the defense programs complex, to give the reader some background 
information on the production of incinerator ash. Section 1.3 summarizes what little data are available in reports and scientific literature concerning the chemistry of plutonium-bearing incinerator ash. Section 1.3 also presents detailed chemical analyses of incinerator ash received at Los Alamos several years ago from Rocky Flats, as well as concomitant analyses of ash and ash heel produced at Los Alamos. A wide variety of experiments were required to investigate the complex chemistry of incinerator ash. Results are presented for weight-loss-on-ignition studies (considered to be a measure of the amount of water and carbon in the material), sieving and particle size fractionation studies, X-ray diffraction studies, infrared spectroscopy studies, volatility studies using fluorination coupled with Fourier transform infrared spectroscopy, electron microprobe studies of virgin ash produced at both Los Alamos and Rocky Flats, electron microprobe studies of Los Alamos and Rocky Flats calcined ash, and electron microprobe studies of ash heel generated at Los Alamos. Part 1 concludes with an overview of ash chemistry and how our new knowledge may lead to new and better processes for recovering plutonium from ash.

\subsection{INCINERATION OF PLUTONIUM-CONTAINING COMBUSTIBLE MATERIALS IN THE DEFENSE PROGRAMS COMPLEX}

\subsubsection{Los Alamos National Laboratory}

Incineration of combustible materials, such as rags and paper, at Los Alamos is well documented. Development work started in 1951 on the design and construction of an incinerator, which began operating in 1952 [MCNEESE-1954, -1960, CHRISTENSEN-1969]. The incinerator design developed in 1952 is that used today in the PF-4 plutonium plant at TA-55 (Los Alamos) [CHRISTENSEN-1988]. In this design, a wire basket is used to contain the items to be incinerated. Oxygen is passed through the burn chamber, and off-gases are pulled by vacuum through a bubbler, condenser, and filter.

Details of the incineration process using the incinerator design reported by Christensen and McNeese [CHRISTENSEN-1969, -1988; MCNEESE-1954, -1960] are described in the Group MST12 standard operating procedure 422-REC-R05 [OLSEN]. Rags, paper wipes, wood, and plastic tape are burned to reduce waste volume. Materials such as thermoplastics and rubber are normally excluded from incineration and are acid-leached to remove plutonium. However, if these materials are not cleaned effectively by leaching, small amounts $(\sim 10 \%$ of a batch) may be incinerated with rags or paper.

When wood is burned, it is broken into small pieces and fed into the incinerator at a slow rate to prevent excessive heat generation during combustion. Rags that are wet with organic solvent, oil, or acid are not accepted for incineration. Rags containing acid must be rinsed in water and dried before incineration. 
Combustion temperatures within the incinerator are not known exactly, but are presumed to be between 200 and $900^{\circ} \mathrm{C}$, depending on the materials being incinerated [CHRISTENSEN1988]. The temperature of the off-gas line at the bottom of the furnace is monitored, however, and is usually between 250 and $350^{\circ} \mathrm{C}$.

Ash is removed from the basket and rod-milled using steel rods. The ash is then sieved through a -16 or -20 mesh screen. Large chunks of ash exhibiting a high gamma count are ground in an iron mortar and then returned for rod-milling. Large particles of glass, glass fibers, or char are removed and are subsequently treated by noncombustible acid leaching.

\subsubsection{Rocky Flats Plant}

Published information concerning incinerator design at Rocky Flats is sparse. Apparently, prior to 1961 a small incinerator was used to produce ash in campaigns run in 1957 and 1958 [BUTLER]. A larger incinerator was installed in 1961. Initial runs in the larger incinerator, and all runs in the smaller incinerator, involved burning polyvinyl chloride (PVC) plastic, polyurethane, rubber gloves, paper, graphite, rags, wood, and ion-exchange resin. Eventually, rubber gloves and PVC plastics were excluded from incineration.

In 1968, Foster presented a brief overview report of incinerators at the various defense program sites [FOSTER]. On the basis of the limited details presented in that report, the Rocky Flats incinerator has a waste feed rate of $20-50 \mathrm{lb} / \mathrm{h}(9.1-22.7 \mathrm{~kg} / \mathrm{h})$. The incinerator is a commercial unit with a rectangular primary combustion chamber and a fluted grate. The incinerator operates by direct gas firing and also contains a secondary combustion chamber with a gas afterburner and baffles. The operating temperature of the incinerator is limited by the thickness of the combustion chamber insulation (refractory brick). This limitation in operating temperature produces a sooty off-gas and leaves a high percentage of unburned material in the ash.

\subsubsection{Hanford Site (200 West Area)}

Foster briefly describes incineration at the Hanford Site [FOSTER]. The incinerator has a feed rate of $10-50 \mathrm{lb} / \mathrm{h}$. It has a continuous belt feeder, is indirectly heated in both the primary and secondary combustion chambers, and is made of metal, which limits the combustion temperature. The incinerator requires shredded feed and removal of tramp metal. Ash is continuously removed from the incinerator. The incinerator design provides close control of residence time for material in the combustion chamber. 


\subsubsection{Savannah River Site}

A $5 \mathrm{~kg} / \mathrm{h}$ controlled-air incinerator has been developed at Savannah River Laboratory to incinerate Savannah River Plant solid transuranic wastes [FREED]. The incinerator design uses prefabricated ceramic components to make up two combustion chambers. Silicon carbide is used in the primary combustion chamber. Supplemental heating is required in the incinerator because of the low exothermicity of the waste being burned.

Shredded waste feed is packaged in cardboard boxes that are dropped directly into the primary combustion chamber. A mixture of air and steam is used to pyrolyze the material between $700^{\circ} \mathrm{C}$ and $900^{\circ} \mathrm{C}$. The ash falls directly into a retention chamber, from which it can be periodically removed.

The Savannah River transuranic waste to be burned in this incinerator is made up of approximately $31 \mathrm{wt} \%$ cellulosic material, $27 \mathrm{wt} \%$ polyvinyl chloride, $21 \mathrm{wt} \%$ polyethylene, and $21 \mathrm{wt} \%$ latex.

\subsection{INCINERATOR ASH AND ASH HEEL CHARACTERIZATION}

\subsubsection{Literature Review}

Little documentation exists concerning the chemistry of incinerator ash generated in the defense programs complex. An early Rocky Flats report described ash used in a dissolution study as containing 0.01 to $0.7 \mathrm{~g} \mathrm{Pu} / \mathrm{g}$ ash sample [BUTLER]. It was noted that "the greater portion of the ash contained from $0.10-0.25 \mathrm{~g} \mathrm{Pu} / \mathrm{g}$ ash." Another Rocky Flats report summarized the chemical composition of typical Rocky Flats incinerator ash (uncalcined) and ash heel (in which it was assumed that all the metals existed as separate binary oxide phases) [JOHNSON]. This information is provided in Table 1.1 .

The most comprehensive description to date of incinerator ash produced at Rocky Flats is given in a Hanford report by Delegard [DELEGARD]. Delegard received three types of Rocky Flats ash, all of which were uncalcined. The ash types were designated as old, middle, and current. The old ash was placed in storage at Rocky Flats on July 17, 1978; the middle ash was placed in storage on January 7, 1980; and the current ash was produced on September 1, 1983. The ash samples had average plutonium contents of $4.1 \mathrm{wt} \%$ (old ash), $6.4 \mathrm{wt} \%$ (middle ash), and $18.2 \mathrm{wt} \%$ (current ash).

Delegard performed ash reburning studies on the three ash types and also measured weightloss-on-ignition (LOD) [DELEGARD]. These studies were performed by heating the ash in a muffle 
furnace in air at $600^{\circ} \mathrm{C}$ for at least $4 \mathrm{~h}$. The old ash lost $23.1 \%$ of its weight during LOI studies, the middle ash lost an average of $9.03 \%$ of its weight, and the current ash lost an average of $2.62 \%$ of its weight.

Delegard [DELEGARD] performed inductively coupled plasma analyses on ash dissolution specimens to obtain information on the chemical composition of the ash and ash heel. These results are summarized in Table 1.2 for the ash and Table 1.3 for the ash heel. The data in Table 1.3 were obtained at two different solids/ liquid ratios (i.e., $100 \mathrm{~g} / \mathrm{L}$ and $200 \mathrm{~g} / \mathrm{L}$ ) during $\mathrm{HNO}_{3}-\mathrm{HF}$ multicontact dissolutions.

\subsubsection{Present Studies}

Emission spectroscopy, loss-on-ignition, and fractionation (sieving) studies were performed on samples of Los Alamos and Rocky Flats virgin and rotary calcined incinerator ash. An additional study was performed on Los Alamos ash to compare several techniques for measuring plutonium content. Studies of the effects of fluorination on residual ash composition were also conducted.

Table 1.4 compares radiochemical analysis, solution assay instrument ( $\mathrm{SAI}$ ), and isotope dilution mass spectrometry (IDMS) results for a virgin Los Alamos ash sample (RGBASH1).

The SAI and IDMS techniques provide values for plutonium content that are in excellent agreement. Radiochemical analysis gives values about 7\% lower than SAI and IDMS. In other studies we observed that radiochemical analysis techniques also exhibit poor precision.

Table 1.5 summarizes the analytical chemistry for three separate batches of virgin Los Alamos incinerator ash. Table 1.6 presents analytical data for three separate batches of virgin Rocky Flat incinerator ash, and Table 1.7 presents analytical data for two of the three batches of virgin

TABLE 1.1 Chemical Composition of Typical Rocky Flats Incinerator Ash and Ash Heel [JOHNSON]

Composition, Wt\%

\begin{tabular}{lrr} 
Constituent & Ash & Ash Heel \\
\hline $\mathrm{Al}_{2} \mathrm{O}_{3}$ & 3.3 & 4.2 \\
$\mathrm{BaO}$ & 0.9 & 0.3 \\
$\mathrm{~B}_{2} \mathrm{O}_{3}$ & 1.8 & 1.6 \\
$\mathrm{CaO}$ & 4.0 & 3.4 \\
$\mathrm{Cr}_{2} \mathrm{O}_{3}$ & 0.7 & 1.7 \\
$\mathrm{CuO}$ & 1.0 & 0.3 \\
$\mathrm{Fe}_{2} \mathrm{O}_{3}$ & 5.7 & 6.2 \\
$\mathrm{PbO}_{\mathrm{MgO}}$ & 0.8 & 0.3 \\
$\mathrm{MnO}_{2}$ & 4.6 & 1.3 \\
$\mathrm{NiO}$ & 0.1 & 0.1 \\
$\mathrm{P}_{2} \mathrm{O}_{3}$ & 0.5 & 0.5 \\
$\mathrm{~K}_{2} \mathrm{O}$ & 0.0 & 1.2 \\
$\mathrm{PuO}_{2}$ & 0.7 & 0.3 \\
$\mathrm{SiO}_{2}$ & 2.8 & 2.5 \\
$\mathrm{Na}_{2} \mathrm{O}$ & 48.5 & 43.0 \\
$\mathrm{Ta}_{2} \mathrm{O}_{3}$ & 1.2 & 0.6 \\
$\mathrm{SnO}_{\mathrm{TiO}_{2}}$ & 0.4 & 0.9 \\
$\mathrm{C}$ & 0.1 & 0.2 \\
$\mathrm{Total}$ & 1.4 & 3.0 \\
\hline & 22.0 & 28.0 \\
\hline & 100.5 & 99.6 \\
\hline
\end{tabular}


TABLE 1.2 Chemical Composition of Rocky Flats Incinerator Ash by Age [DELEGARD]

\begin{tabular}{lccc}
\hline & \multicolumn{3}{c}{ Composition, Wt\% } \\
\cline { 2 - 4 } Component & Current $^{\mathrm{a}}$ & Middle $^{\mathrm{b}}$ & Old $^{\mathrm{c}}$ \\
\hline $\mathrm{Al}_{2} \mathrm{O}_{3}$ & 13.2 & 32.6 & 3.8 \\
$\mathrm{BaO}$ & 1.9 & 1.5 & 2.3 \\
$\mathrm{~B}_{2} \mathrm{O}_{3}$ & 3.8 & 3.9 & 2.9 \\
$\mathrm{CaO}$ & 22.2 & 19.6 & 7.4 \\
$\mathrm{Cr}_{2} \mathrm{O}_{3}$ & 1.5 & 1.8 & - \\
$\mathrm{Fe}_{2} \mathrm{O}_{3}$ & 9.4 & 14.0 & 6.0 \\
$\mathrm{PbO}_{2}$ & -- & - & 59.0 \\
$\mathrm{MgO}_{\mathrm{SiO}}$ & 5.1 & 4.4 & 12.8 \\
$\mathrm{TiO}_{2}$ & 23.8 & 24.7 & 18.1 \\
$\mathrm{ZnO}_{\mathrm{PuO}_{2}}$ & 2.7 & 3.2 & -- \\
$\mathrm{Total}$ & 2.6 & 2.2 & 2.6 \\
\cline { 2 - 4 } & 23.2 & 9.5 & 6.5 \\
\hline
\end{tabular}

a Produced September 1, 1983.

b Stored January 7, 1980.

c Stored July 17, 1978.

Rocky Flats incinerator ash that were rotary calcined. Table 1.8 summarizes LOI data on virgin and calcined ash samples, while Table 1.9 presents results from a sieving fractionation study performed on both Los Alamos and Rocky Flats incinerator ash.

The data in Tables 1.5, 1.6, and 1.7 show remarkable similarities in the chemical composition of Los Alamos and Rocky Flats ashes. Also, calcination does not appear to alter the chemical composition of the ash other than to burn off free carbon, remove adhered water, and perhaps remove molybdenum as the volatile $\mathrm{MoO}_{3}$ or $\mathrm{MoO}_{3}$ hydrate. According to Tables 1.5, 1.6, and 1.7, the major ( $1 \%$ or greater) chemical components of the ashes studied are $\mathrm{Al}, \mathrm{C}, \mathrm{Ca}, \mathrm{Cl}, \mathrm{Cr}$, $\mathrm{Fe}, \mathrm{K}, \mathrm{Mg}, \mathrm{Mn}, \mathrm{Mo}, \mathrm{Na}, \mathrm{Ni}, \mathrm{Si}$, and $\mathrm{Zn}$. The only major difference between the Rocky Flats and Los Alamos ashes was that $\mathrm{Pb}, \mathrm{Ti}$, and $\mathrm{Ba}$ were observed in the Rocky Flats ash samples. The data provided in Tables 1.5 and 1.6 are in remarkable agreement with the chemical composition reported by Johnson (Table 1.1) for a sample of virgin Rocky Flats ash in 1976 [JOHNSON]. 
TABLE 1.3 Chemical Composition of Rocky Flats Incinerator Ash Heel by Age and Ash-to-Leachate Ratio [DELEGARD]

\begin{tabular}{|c|c|c|c|c|c|}
\hline \multirow[b]{3}{*}{ Component } & \multicolumn{5}{|c|}{ Composition, Wt\% } \\
\hline & \multicolumn{2}{|c|}{ Current $^{a}$} & \multicolumn{2}{|c|}{ Middle. } & \multirow{2}{*}{$\frac{\mathrm{Old}^{\mathrm{c}}}{200 \mathrm{~g} / \mathrm{L}}$} \\
\hline & $100 \mathrm{~g} / \mathrm{L}^{\mathrm{d}}$ & $200 \mathrm{~g} / \mathrm{L}$ & $100 \mathrm{~g} / \mathrm{L}$ & $200 \mathrm{~g} / \mathrm{L}$ & \\
\hline $\mathrm{Al}_{2} \mathrm{O}_{3}$ & 14.1 & 17.6 & 36.7 & 46.8 & -- \\
\hline $\mathrm{BaO}$ & 1.1 & 1.1 & 1.0 & 1.0 & 2.7 \\
\hline $\mathrm{B}_{2} \mathrm{O}_{3}$ & 2.8 & 3.1 & -- & -- & 2.4 \\
\hline $\mathrm{CaF}_{2}$ & 77.2 & 60.0 & 60.6 & 28.0 & 24.3 \\
\hline $\mathrm{Cr}_{2} \mathrm{O}_{3}$ & 2.5 & 4.3 & 1.5 & 1.9 & -- \\
\hline $\mathrm{Fe}_{2} \mathrm{O}_{3}$ & 5.2 & 8.4 & 4.3 & 11.4 & 2.3 \\
\hline $\mathrm{PbO}_{2}$ & - & -- & -- & -- & 60.1 \\
\hline $\mathrm{MgO}$ & 2.9 & 2.4 & 2.4 & 2.4 & 2.0 \\
\hline $\mathrm{SiO}_{2}$ & 8.2 & 9.4 & 13.6 & 16.5 & -- \\
\hline $\mathrm{TiO}_{2}$ & 1.6 & 3.2 & 2.1 & 4.3 & -- \\
\hline $\mathrm{ZnO}$ & 2.1 & 1.9 & 1.7 & 1.8 & 1.4 \\
\hline $\mathrm{PuO}_{2}$ & 0.8 & 0.4 & 0.6 & 0.8 & 0.3 \\
\hline Total & 118.5 & 111.8 & 124.5 & 114.9 & 95.5 \\
\hline
\end{tabular}

a Produced September 1, 1983.

b Stored January 7, 1980.

c Stored July 17, 1978.

d Grams of ash per liter of leachant.

TABLE 1.4 Comparison of Plutonium Content of Virgin Los Alamos Incinerator Ash ${ }^{\mathbf{a}}$ as Measured by Several Techniques

\begin{tabular}{lc}
\hline \multicolumn{1}{c}{ Technique } & $\begin{array}{c}\text { Plutonium } \\
\text { Content, Wt\% }\end{array}$ \\
\hline Radiochemical analysis & 12.8 \\
Solution assay instrument & 13.8 \\
Isotope dilution mass spectrometry & $13.8,13.6,13.5$ \\
\hline
\end{tabular}

a Batch No.: RGBASH1. 
TABLE 1.5 Elemental Analyses

for Three Batches of Virgin

Los Alamos Incinerator Ash

\begin{tabular}{|c|c|c|c|}
\hline \multirow[b]{2}{*}{ Element } & \multicolumn{3}{|c|}{ Composition ${ }^{\mathrm{a}}$} \\
\hline & BASH1 & BASH2 & LSA \\
\hline $\mathrm{Ag}$ & 100 & 1000 & $<30$ \\
\hline $\mathrm{Al}$ & 5000 & $2 \%$ & $6 \%$ \\
\hline $\mathrm{B}$ & 400 & 300 & 500 \\
\hline $\mathrm{Ba}$ & 500 & 1500 & 2000 \\
\hline $\mathrm{Be}$ & 10 & 10 & 200 \\
\hline $\mathrm{Bi}$ & $<100$ & $<100$ & 400 \\
\hline C & $1.1 \%$ & $1.8 \%$ & -.- \\
\hline $\mathrm{Ca}$ & $20 \%$ & $5 \%$ & $6 \%$ \\
\hline $\mathrm{Cd}$ & 1000 & 3000 & $<100$ \\
\hline $\mathrm{Ce}$ & $<500$ & $<500$ & $<1000$ \\
\hline $\mathrm{Cl}$ & $17.6 \%$ & $9.80 \%$ & --- \\
\hline Co & $<100$ & $<100$ & $<300$ \\
\hline $\mathrm{Cr}$ & $2 \%$ & $3 \%$ & $4 \%$ \\
\hline $\mathrm{Cu}$ & 5000 & $1.5 \%$ & $1.5 \%$ \\
\hline $\mathrm{Fe}$ & $4 \%$ & $15 \%$ & $10 \%$ \\
\hline $\mathrm{Ga}$ & 3000 & $2 \%$ & $<100$ \\
\hline $\mathrm{Ge}$ & $<100$ & $<100$ & $<100$ \\
\hline$H$ & 4900 & 3000 & --- \\
\hline $\mathrm{Hf}$ & $<300$ & $<300$ & $<1000$ \\
\hline $\mathrm{K}$ & $20 \%$ & $5 \%$ & $8 \%$ \\
\hline $\mathrm{La}$ & $<300$ & --- & $<300$ \\
\hline $\mathrm{Li}$ & 2000 & 3000 & $<300$ \\
\hline $\mathrm{Mg}$ & $5 \%$ & $7 \%$ & $4 \%$ \\
\hline $\mathrm{Mn}$ & 5000 & $2 \%$ & 6000 \\
\hline Mo & 4000 & $1 \%$ & $1 \%$ \\
\hline $\mathrm{Na}$ & $5 \%$ & $5 \%$ & $5 \%$ \\
\hline $\mathrm{Nb}$ & $<300$ & $<300$ & $<500$ \\
\hline $\mathrm{Ni}$ & $1 \%$ & $3 \%$ & $2 \%$ \\
\hline$P$ & $3 \%$ & $2 \%$ & $\cdots$ \\
\hline $\mathrm{Pb}$ & 500 & 3000 & 5000 \\
\hline $\mathrm{Pu}$ & $12.8 \%$ & $17.7 \%$ & $17.3 \%$ \\
\hline $\mathrm{Rb}$ & $<1000$ & --- & ... \\
\hline $\operatorname{Re}$ & $<1000$ & $\ldots$ & $<1000$ \\
\hline$S$ & 1900 & --- & --- \\
\hline $\mathrm{Sb}$ & $<300$ & --- & $<500$ \\
\hline $\mathrm{Si}$ & $2 \%$ & $10 \%$ & $5 \%$ \\
\hline $\mathrm{Sn}$ & $<300$ & $<300$ & $<3000$ \\
\hline $\mathrm{Sr}$ & 2000 & 1000 & 5000 \\
\hline $\mathrm{Ta}$ & $<3000$ & $<3000$ & $<3000$ \\
\hline $\mathrm{Ti}$ & 2000 & 5000 & 6000 \\
\hline $\mathrm{V}$ & $<100$ & $<100$ & 100 \\
\hline W & $<3000$ & $<3000$ & $<3000$ \\
\hline $\mathrm{Y}$ & 1000 & 500 & $<500$ \\
\hline $\mathrm{Zn}$ & 1000 & $1.5 \%$ & 5000 \\
\hline $\mathrm{Zr}$ & $<100$ & $<100$ & $<300$ \\
\hline
\end{tabular}

a Values represent $\mathrm{mg} / \mathrm{kg}$ unless otherwise indicated. All data are Spectro Quant results with the exception of $\mathrm{C}, \mathrm{H}, \mathrm{Cl}, \mathrm{S}$, and $\mathrm{Pu}$.

The data thus have a factor of two accuracy and a precision of $50 \%$ rsd.
TABLE 1.6 Elemental Analyses for Three Batches of Virgin Rocky Flats Incinerator Ash

\begin{tabular}{|c|c|c|c|}
\hline \multirow[b]{2}{*}{ Element } & \multicolumn{3}{|c|}{ Composition $^{\mathrm{a}}$} \\
\hline & BARF1 & BARF2 & BARF3 \\
\hline $\mathrm{Ag}$ & 500 & $<30$ & $<30$ \\
\hline $\mathrm{Al}$ & $5 \%$ & $5 \%$ & $6 \%$ \\
\hline B & 1000 & 2000 & 500 \\
\hline $\mathrm{Ba}$ & $2 \%$ & $1.5 \%$ & $0.2 \%$ \\
\hline $\mathrm{Be}$ & 1500 & 3000 & 200 \\
\hline $\mathrm{Bi}$ & $<100$ & $<100$ & 400 \\
\hline $\mathrm{C}$ & $8 \%$ & $7 \%$ & $\ldots$ \\
\hline $\mathrm{Ca}$ & $15 \%$ & $8 \%$ & $6 \%$ \\
\hline $\mathrm{Cd}$ & 1000 & 500 & $<100$ \\
\hline $\mathrm{Ce}$ & $<500$ & $<500$ & $<1000$ \\
\hline $\mathrm{Cl}$ & $2.50 \%$ & $3.30 \%$ & --- \\
\hline Co & $<100$ & $<100$ & $<300$ \\
\hline $\mathrm{Cr}$ & $3 \%$ & $2 \%$ & $4 \%$ \\
\hline $\mathrm{Cu}$ & $1.5 \%$ & $2 \%$ & $1.5 \%$ \\
\hline $\mathrm{Fe}$ & $15 \%$ & $5 \%$ & $10 \%$ \\
\hline $\mathrm{Ga}$ & 2000 & 500 & $<100$ \\
\hline $\mathrm{Ge}$ & $<100$ & $<100$ & $<100$ \\
\hline $\mathrm{H}$ & 6000 & 5000 & --- \\
\hline Hf & $<300$ & $<300$ & $<1000$ \\
\hline $\mathbf{K}$ & $3 \%$ & $20 \%$ & $8 \%$ \\
\hline La & $\ldots$ &.-- & $<300$ \\
\hline $\mathrm{Li}$ & 500 & $<300$ & $<300$ \\
\hline $\mathrm{Mg}$ & $10 \%$ & $3 \%$ & $4 \%$ \\
\hline $\mathrm{Mn}$ & $3 \%$ & $1 \%$ & 6000 \\
\hline Mo & 5000 & $<300$ & $1 \%$ \\
\hline $\mathrm{Na}$ & $3 \%$ & $2 \%$ & $5 \%$ \\
\hline $\mathrm{Nb}$ & $<300$ & $<300$ & $<500$ \\
\hline $\mathrm{Ni}$ & $3 \%$ & $2 \%$ & $2 \%$ \\
\hline $\mathrm{P}$ & $<3000$ & $<3000$ & --- \\
\hline $\mathrm{Pb}$ & $2 \%$ & $4 \%$ & 5000 \\
\hline $\mathrm{Pu}$ & $13.8 \%$ & $8.62 \%$ & $17.3 \%$ \\
\hline $\mathrm{Rb}$ & --- & --- & --- \\
\hline $\operatorname{Re}$ & --- & --- & $<1000$ \\
\hline$S$ & $\cdots$ & -- & --- \\
\hline $\mathrm{Sb}$ & --- & -.- & $<500$ \\
\hline $\mathrm{Si}$ & $20 \%$ & $15 \%$ & $5 \%$ \\
\hline Sn & $<300$ & 4000 & $<3000$ \\
\hline $\mathrm{Sr}$ & 2000 & 3000 & 5000 \\
\hline $\mathrm{Ta}$ & $<3000$ & $<3000$ & $<3000$ \\
\hline $\mathrm{Ti}$ & $5 \%$ & $5 \%$ & 6000 \\
\hline $\mathrm{V}$ & $<100$ & $<100$ & 100 \\
\hline W & $<3000$ & $<3000$ & $<3000$ \\
\hline$Y$ & 500 & $<100$ & $<500$ \\
\hline $\mathrm{Zn}$ & $4 \%$ & $3 \%$ & 5000 \\
\hline $\mathrm{Zr}$ & 5000 & $<100$ & $<300$ \\
\hline $\begin{array}{l}\text { Values } \\
\text { indicat } \\
\text { with th } \\
\text { The dat } \\
\text { and a p }\end{array}$ & $\begin{array}{l}\text { ent mg } \\
\text { ll data ar } \\
\text { eption of } \\
s \text { have a } \\
\text { ion of } 50\end{array}$ & $\begin{array}{l}\text { nless othe } \\
\mathrm{H}, \mathrm{Cl}, \mathrm{S} \text {, } \\
\text { or of two } \\
\text { d. }\end{array}$ & $\begin{array}{l}\text { vise } \\
\text { results } \\
\mathrm{Pu} \text {. } \\
\text { curacy }\end{array}$ \\
\hline
\end{tabular}


Table 1.8 gives the results of LOI tests. The larger weight loss of virgin ash compared to calcined ash suggests that more complete burning is achieved during the calcination process. Mass spectrometry data obtained during oxidation of virgin ash and obtained during LOI experiments show that the major vapor species are hydrogen, $\mathrm{CO}$, methane, water, $\mathrm{CO}_{2}$, oxygen, and perhaps some $\mathrm{SO}$ and $\mathrm{SO}_{2}$. Chlorine-containing vapor species were not observed, indicating that chlorine is not removed as chlorine-oxygen or chlorinefluoride vapor species. That chlorine is contained in the ash during calcination is confirmed by ancillary analytical data on virgin ash and calcined ash (see Tables 1.6 and 1.7).

Results from sieving experiments shown in Table 1.9 indicate that no major fractionation of plutonium occurs during simple sieving experiments. Thus, concentrating the plutonium in a small-particle-size fraction of the ash does not appear feasible without prior physical treatment such as grinding.

Tables 1.10-1.15 present analytical results for the filter residues and ash heels obtained from six small-scale cascade dissolver dissolution studies performed on calcined Rocky Flats ash (samples BARF1RC and BARF2RC). Sodium, potassium, chlorine, lead, and plutonium are preferentially removed from the ash by dissolution. Analytical data on the filtrate from the dissolvers confirm these results for sodium, potassium, and chlorine. Also, iron appears to be dissolved preferentially according to the filtrate analytical results. However, lead does not appear in any significant quantities in the filtrate. All other major metals present in the ash are also present in the ash filter residue and ash heel to about the same extent.
TABLE 1.7 Elemental Analyses for Two Batches of Calcined Rocky Flats Incinerator Ash

\begin{tabular}{|c|c|c|}
\hline \multirow[b]{2}{*}{ Element } & \multicolumn{2}{|c|}{ Composition $^{\mathrm{a}}$} \\
\hline & BARFI & BARF2 \\
\hline $\mathrm{Ag}$ & $<100$ & $<100$ \\
\hline $\mathrm{Al}$ & $5 \%$ & $5 \%$ \\
\hline B & 1000 & 1000 \\
\hline $\mathrm{Ba}$ & $0.8 \%$ & $1 \%$ \\
\hline $\mathrm{Be}$ & 2000 & 4000 \\
\hline $\mathrm{Bi}$ & $<100$ & $<100$ \\
\hline C & 2600 & 3200 \\
\hline $\mathrm{Ca}$ & $6 \%$ & $8 \%$ \\
\hline $\mathrm{Cd}$ & 500 & 400 \\
\hline $\mathrm{Ce}$ & $<500$ & $<500$ \\
\hline $\mathrm{Cl}$ & $2.9 \%$ & $3.30 \%$ \\
\hline Co & $<100$ & $<100$ \\
\hline $\mathrm{Cr}$ & $2 \%$ & $2 \%$ \\
\hline $\mathrm{Cu}$ & 7000 & $1 \%$ \\
\hline $\mathrm{Fe}$ & $5 \%$ & $5 \%$ \\
\hline $\mathrm{Ga}$ & 2000 & 2000 \\
\hline $\mathrm{Ge}$ & $<100$ & $<100$ \\
\hline $\mathrm{H}$ & 1600 & 870 \\
\hline $\mathrm{Hf}$ & $<500$ & $<500$ \\
\hline$K$ & $5 \%$ & $10 \%$ \\
\hline La & --- & -- \\
\hline $\mathrm{Li}$ & 300 & 200 \\
\hline $\mathrm{Mg}$ & $1 \%$ & $1 \%$ \\
\hline $\mathrm{Mn}$ & 7000 & 5000 \\
\hline Mo & 4000 & 2000 \\
\hline $\mathrm{Na}$ & $1.5 \%$ & $1.5 \%$ \\
\hline $\mathrm{Nb}$ & $<300$ & $<300$ \\
\hline $\mathrm{Ni}$ & $1.5 \%$ & $2 \%$ \\
\hline $\mathrm{P}$ & $<3000$ & 5000 \\
\hline $\mathrm{Pb}$ & $1 \%$ & $2 \%$ \\
\hline $\mathrm{Pu}$ & $14.5 \%$ & $9.07 \%$ \\
\hline $\mathrm{Rb}$ & -. & -- \\
\hline $\operatorname{Re}$ & --- & --- \\
\hline$S$ & --- & -- \\
\hline $\mathrm{Sb}$ & -- & $\cdots$ \\
\hline $\mathrm{Si}$ & $7 \%$ & $10 \%$ \\
\hline Sn & $<300$ & $<300$ \\
\hline $\mathrm{Sr}$ & 1000 & 1000 \\
\hline $\mathrm{Ta}$ & $<3000$ & $<3000$ \\
\hline $\mathrm{Ti}$ & $3 \%$ & $4 \%$ \\
\hline V & $<100$ & $<100$ \\
\hline w & $<300$ & $<3000$ \\
\hline$Y$ & 1000 & 100 \\
\hline $\mathrm{Zn}$ & $1 \%$ & $2 \%$ \\
\hline $\mathrm{Zr}$ & 200 & 500 \\
\hline
\end{tabular}

a Values represent $\mathrm{mg} / \mathrm{kg}$ unless otherwise indicated. All data are Spectro Quant results with the exception of $\mathrm{C}, \mathrm{H}, \mathrm{Cl}, \mathrm{S}$, and $\mathrm{Pu}$ The data thus have a factor of two accuracy and a precision of $50 \%$ rsd. 
TABLE 1.8 Summary of Loss-On-Ignition (LOI) Data for Incinerator Ash Samples

\begin{tabular}{lrrrc}
\hline & BARF1 & BARF2 & BARF3 & BASH \\
\hline Virgin ash & $10.7 \%$ & $9.7 \%$ & $6.5 \%$ & $11.3 \%$ \\
Calcined ash & $2.1 \%$ & $1.7 \%$ & --- & --- \\
\hline
\end{tabular}

TABLE 1.9 Summary of Sieving Studies on Incinerator Ash Samples

\begin{tabular}{|c|c|c|c|}
\hline $\begin{array}{c}\text { Size } \\
\text { Fraction, } \\
\text { Mesh }\end{array}$ & $\begin{array}{c}\text { Particle-Size } \\
\text { Distribution, } \\
\text { Wt\% }\end{array}$ & $\begin{array}{c}{[\mathrm{Pu}]} \\
\mathrm{g} / \mathrm{g}\end{array}$ & $\begin{array}{c}\text { Plutonium } \\
\text { Distribution, } \\
\text { Wt } \%\end{array}$ \\
\hline \multicolumn{4}{|c|}{ Sample: BASH (Virgin, Los Alamos Ash) } \\
\hline$>45$ & 47.9 & 0.125 & 47.9 \\
\hline $45-80$ & 26.1 & 0.100 & 20.9 \\
\hline $80-170$ & 20.0 & 0.135 & 21.6 \\
\hline$<170$ & 6.0 & 0.204 & 9.8 \\
\hline \multicolumn{4}{|c|}{ Sample: BARF1 (Virgin, Rocky Flats Ash) } \\
\hline$>45$ & 61.8 & 0.128 & 59.9 \\
\hline $45-80$ & 23.0 & 0.134 & 23.3 \\
\hline $80-170$ & 12.1 & 0.141 & 12.9 \\
\hline$<170$ & 3.1 & 0.152 & 3.6 \\
\hline \multicolumn{4}{|c|}{ Sample: BARF2 (Virgin, Rocky Flats Ash) } \\
\hline$>45$ & 2.1 & 0.069 & 1.7 \\
\hline $45-80$ & 42.7 & 0.081 & 40.7 \\
\hline $80-170$ & 43.2 & 0.087 & 44.2 \\
\hline$<170$ & 12.1 & 0.098 & 13.9 \\
\hline \multicolumn{4}{|c|}{ Sample: BARF3 (Virgin, Rocky Flats Ash) } \\
\hline$>45$ & 65.3 & 0.138 & 60.9 \\
\hline $45-80$ & 18.9 & 0.145 & 18.5 \\
\hline $80-170$ & 10.9 & 0.172 & 12.7 \\
\hline$<170$ & 4.9 & 0.236 & 7.8 \\
\hline
\end{tabular}


TABLE 1.9 (Cont.)

\begin{tabular}{cccc}
\hline $\begin{array}{c}\text { Size } \\
\text { Fraction, } \\
\text { Mesh }\end{array}$ & $\begin{array}{c}\text { Particle-Size } \\
\text { Distribution, } \\
\text { Wt\% }\end{array}$ & $\begin{array}{c}\text { [Pu], } \\
\text { g/g }\end{array}$ & $\begin{array}{c}\text { Plutonium } \\
\text { Distribution, } \\
\text { Wt\% }\end{array}$ \\
\hline \multicolumn{4}{c}{ Sample: BARF1RC } \\
(Rotary-Calcined Rocky
\end{tabular}

The analytical results also show that no major chemistry differences associated with the major metals and chlorine are present as a result of the calcination step. This was also confirmed by electron microprobe studies of virgin and calcined ash samples and by mass spectrometric studies performed at Rocky Flats.

\subsubsection{Fluorination Studies}

A 240.7 -g batch of virgin Los Alamos ash (RGBASH1) was fluorinated at $250^{\circ} \mathrm{C}$ with $\mathrm{F}_{2}$. By DMS, the material had $15.0 \mathrm{wt} \%$ plutonium. After fluorination, the material was passed through four sieves. For analysis, the two finest sieved fractions were combined and the two coarsest sieved fractions were combined. Table 1.16 shows the results of the analysis.

A second 254.7-g lot of virgin Los Alamos incinerator ash (RGBASH1) was treated with $\mathrm{F}_{2}$ at $250^{\circ} \mathrm{C}$. The final weight of the material was $250.7 \mathrm{~g}$. The plutonium content of the fluorinated ash was $14.0 \mathrm{wt} \%$ by IDMS and $14.2 \mathrm{wt} \%$ by SAI (average of three runs). Using FTIR spectroscopy, it was determined that $5.2 \mathrm{~g}(2.04 \mathrm{wt} \%)$ of $\mathrm{C}$ was volatilized mainly as $\mathrm{CO}_{2}$ and $\mathrm{CF}_{4} ; 1.38 \mathrm{~g}$ $(0.51 \mathrm{wt} \%)$ of $\mathrm{Si}$ was volatilized as $\mathrm{SiF}_{4} ; 2.6 \mathrm{~g}(1.02 \%)$ of $\mathrm{S}$ was lost as $\mathrm{SO}_{2} \mathrm{~F}_{2}$; and $4.0 \mathrm{~g}(1.60 \mathrm{wt} \%)$ of $\mathrm{P}$ was lost as $\mathrm{PF}_{5}$ and $\mathrm{POF}_{3}$. Table 1.17 summarizes the analytical results for the condensed material after fluorination. 
TABLE 1.10 Elemental Composition of Filter Residue and Dissolver Heel Obtained during Small-Scale Cascade Dissolution Studies of Calcined Rocky Flats Ash in $\mathrm{HNO}_{3} / \mathrm{HF}$; Experiment $1^{\text {a }}$

\begin{tabular}{|c|c|}
\hline \multirow[b]{2}{*}{ Element } & Composition, $\mathrm{mg} / \mathrm{kg}$ \\
\hline & Filter Residue + Dissolver Heel \\
\hline $\mathrm{Ag}$ & $<100$ \\
\hline Al & 30,000 \\
\hline B & 1000 \\
\hline $\mathrm{Ba}$ & 3000 \\
\hline $\mathrm{Be}$ & 400 \\
\hline $\mathrm{Bi}$ & $<100$ \\
\hline C & 4000 \\
\hline $\mathrm{Ca}$ & 20,000 \\
\hline $\mathrm{Cd}$ & 100 \\
\hline $\mathrm{Ce}$ & $<500$ \\
\hline $\mathrm{Cl}$ & 1300 \\
\hline $\mathrm{Co}$ & $<100$ \\
\hline $\mathrm{Cr}$ & 20,000 \\
\hline $\mathrm{Cu}$ & 3000 \\
\hline $\mathrm{F}$ & 22,000 \\
\hline $\mathrm{Fe}$ & 50,000 \\
\hline $\mathrm{Ga}$ & 2000 \\
\hline $\mathrm{Ge}$ & $<100$ \\
\hline $\mathrm{H}$ & 2700 \\
\hline Hf & $<500$ \\
\hline $\mathrm{K}$ & 8000 \\
\hline $\mathbf{L i}$ & 100 \\
\hline $\mathrm{Mg}$ & 10,000 \\
\hline $\mathrm{Mn}$ & 5000 \\
\hline Mo & 2000 \\
\hline $\mathrm{Na}$ & 2000 \\
\hline $\mathrm{Nb}$ & $<300$ \\
\hline $\mathrm{Ni}$ & 20,000 \\
\hline $\mathrm{P}$ & $<3000$ \\
\hline $\mathrm{Pb}$ & 3000 \\
\hline $\mathrm{Pu}$ & 42,000 \\
\hline $\mathrm{Si}$ & 100,000 \\
\hline $\mathrm{Sn}$ & $<300$ \\
\hline $\mathrm{Sr}$ & 1000 \\
\hline $\mathrm{Ta}$ & $<3000$ \\
\hline $\mathrm{Ti}$ & 40,000 \\
\hline $\mathrm{Tl}$ & $<300$ \\
\hline V & $<100$ \\
\hline$W$ & $<3000$ \\
\hline $\mathrm{Y}$ & 100 \\
\hline $\mathrm{Zn}$ & 10,000 \\
\hline $\mathrm{Zr}$ & 3000 \\
\hline
\end{tabular}

a Ash sample used was BARF1RC. In this experiment only, the dissolver heel and filter residue were combined and then analyzed.
TABLE 1.11 Elemental Composition of Filter Residue and Dissolver Heel Obtained during Small-Scale Cascade Dissolution Studies of Calcined Rocky Flats Ash in $\mathrm{HNO}_{3} / \mathrm{HF}$; Experiment $2^{\mathrm{a}}$

\begin{tabular}{|c|c|c|}
\hline \multirow[b]{2}{*}{ Element } & \multicolumn{2}{|c|}{ Composition, $\mathrm{mg} / \mathrm{kg}$} \\
\hline & Filter Residue & Dissolver Heel \\
\hline $\mathrm{Ag}$ & 500 & 100 \\
\hline $\mathrm{Al}$ & 20,000 & 4000 \\
\hline $\mathrm{B}$ & 500 & 500 \\
\hline $\mathrm{Ba}$ & 4000 & 3000 \\
\hline $\mathrm{Be}$ & 300 & 200 \\
\hline $\mathrm{Bi}$ & $<100$ & $<100$ \\
\hline C & 3500 & 4500 \\
\hline $\mathrm{Ca}$ & 30,000 & 20,000 \\
\hline $\mathrm{Cd}$ & 100 & 200 \\
\hline $\mathrm{Ce}$ & $<500$ & $<500$ \\
\hline $\mathrm{Cl}$ & 670 & 940 \\
\hline $\mathrm{Co}$ & $<300$ & $<100$ \\
\hline $\mathrm{Cr}$ & 30,000 & 20,000 \\
\hline $\mathrm{Cu}$ & 5000 & 3000 \\
\hline F & 16,000 & 19,000 \\
\hline $\mathrm{Fe}$ & 50,000 & 50,000 \\
\hline $\mathrm{Ga}$ & 300 & 500 \\
\hline $\mathrm{Ge}$ & $<100$ & $<100$ \\
\hline $\mathrm{H}$ & 18,000 & 4500 \\
\hline Hf & $<300$ & $<300$ \\
\hline $\mathrm{K}$ & 7000 & 5000 \\
\hline $\mathrm{Li}$ & 100 & 100 \\
\hline $\mathrm{Mg}$ & 10,000 & 15,000 \\
\hline $\mathrm{Mn}$ & 6000 & 4000 \\
\hline Mo & 1000 & 2000 \\
\hline $\mathrm{Na}$ & 2000 & 1500 \\
\hline $\mathrm{Nb}$ & $<300$ & $<300$ \\
\hline $\mathrm{Ni}$ & 20,000 & 20,000 \\
\hline $\mathbf{P}$ & $<3000$ & 3000 \\
\hline $\mathrm{Pb}$ & 3000 & 2000 \\
\hline Pu & 9.09 & 14.5 \\
\hline $\mathrm{Si}$ & 150,000 & 100,000 \\
\hline Sn & $<300$ & $<300$ \\
\hline $\mathrm{Sr}$ & 200 & 200 \\
\hline $\mathrm{Ta}$ & $<3000$ & $<3000$ \\
\hline $\mathrm{Ti}$ & 30,000 & 50,000 \\
\hline $\mathrm{TI}$ & $<300$ & -- \\
\hline $\mathrm{V}$ & $<100$ & $<100$ \\
\hline w & $<3000$ & $<3000$ \\
\hline $\mathrm{Y}$ & 20 & $<100$ \\
\hline $\mathrm{Zn}$ & 5000 & 3000 \\
\hline $\mathrm{Zr}$ & 200 & 4000 \\
\hline
\end{tabular}

a Ash sample used was BARFIRC. 
TABLE 1.12 Elemental Composition of Filter Residue and Dissolver Heel Obtained during Small-Scale Cascade Dissolution Studies of Calcined Rocky Flats Ash in $\mathrm{HNO}_{3} / \mathrm{HF}$; Experiment $3^{\mathrm{a}}$

\begin{tabular}{|c|c|c|}
\hline \multirow[b]{2}{*}{ Element } & \multicolumn{2}{|c|}{ Composition, $\mathrm{mg} / \mathrm{kg}$} \\
\hline & Filter Residue & Dissolver Heel \\
\hline $\mathrm{Ag}$ & 500 & $-\cdots$ \\
\hline Al & 15,000 & --- \\
\hline B & 800 & $\cdots$ \\
\hline $\mathrm{Ba}$ & 4000 & $\cdots$ \\
\hline $\mathrm{Be}$ & 200 & ---- \\
\hline $\mathrm{Bi}$ & $<100$ & $-\ldots-$ \\
\hline $\mathrm{C}$ & 3500 & 4500 \\
\hline $\mathrm{Ca}$ & 20,000 & $\ldots$ \\
\hline $\mathrm{Cd}$ & 100 & ---- \\
\hline $\mathrm{Ce}$ & $<500$ & --- \\
\hline $\mathrm{Cl}$ & 945 & $\ldots$ \\
\hline Co & $<300$ & $\ldots$ \\
\hline $\mathrm{Cr}$ & 20,000 & $\cdots$ \\
\hline $\mathrm{Cu}$ & 4000 & $-\ldots$ \\
\hline $\mathrm{F}$ & 16,000 & ---- \\
\hline $\mathrm{Fe}$ & 50,000 & $\ldots$ \\
\hline $\mathrm{Ga}$ & 300 & --- \\
\hline $\mathrm{Ge}$ & $<100$ & $-\cdots$ \\
\hline $\mathrm{H}$ & --- & $\cdots$ \\
\hline Hf & $<300$ & --- \\
\hline K & 4000 & $\cdots$ \\
\hline $\mathrm{Li}$ & $<100$ & $\cdots$ \\
\hline $\mathrm{Mg}$ & 10,000 & $\ldots$ \\
\hline $\mathrm{Mn}$ & 5000 & --- \\
\hline Mo & 1000 & $\cdots$ \\
\hline $\mathrm{Na}$ & 1000 & $\cdots$ \\
\hline $\mathrm{Nb}$ & $<300$ & --- \\
\hline $\mathrm{Ni}$ & 20,000 & --- \\
\hline $\mathbf{P}$ & $<3000$ & ---- \\
\hline $\mathrm{Pb}$ & 3000 & $\cdots$ \\
\hline $\mathrm{Pu}$ & 99,800 & 62,100 \\
\hline $\mathrm{Si}$ & 80,000 & ...- \\
\hline $\mathrm{Sn}$ & $<300$ & ---- \\
\hline $\mathrm{Sr}$ & 200 & $\ldots$ \\
\hline $\mathrm{Ta}$ & $<3000$ & .... \\
\hline $\mathrm{Ti}$ & 20,000 & ---- \\
\hline $\mathrm{Tl}$ & $<300$ & ---- \\
\hline $\mathrm{V}$ & $<100$ & $\ldots$ \\
\hline W & $<3000$ & ---- \\
\hline$Y$ & 30 & ---- \\
\hline $\mathrm{Zn}$ & 5000 & ---- \\
\hline $\mathrm{Zr}$ & 200 & $\ldots$ \\
\hline
\end{tabular}

a Ash sample used was BARF1RC.
TABLE 1.13 Elemental Composition of Filter Residue and Dissolver Heel Obtained during Small-Scale Cascade Dissolution Studies of Calcined Rocky Flats Ash in $\mathrm{HNO}_{3} / \mathrm{HF}$; Experiment $4^{\mathrm{a}}$

\begin{tabular}{|c|c|c|}
\hline \multirow[b]{2}{*}{ Element } & \multicolumn{2}{|c|}{ Composition, mg $/ \mathrm{kg}$} \\
\hline & Filter Residue & Dissolver Heel \\
\hline $\mathrm{Ag}$ & 500 & 500 \\
\hline $\mathrm{Al}$ & 30,000 & 50,000 \\
\hline B & 1000 & 500 \\
\hline $\mathrm{Ba}$ & 15,000 & 10,000 \\
\hline $\mathrm{Be}$ & 200 & 300 \\
\hline $\mathbf{B i}$ & $<100$ & $<100$ \\
\hline $\mathrm{C}$ & 6000 & 5500 \\
\hline $\mathrm{Ca}$ & 20,000 & 100,000 \\
\hline $\mathrm{Cd}$ & 100 & 100 \\
\hline $\mathrm{Ce}$ & $<500$ & $<500$ \\
\hline $\mathrm{Cl}$ & 645 & 970 \\
\hline $\mathrm{Co}$ & $<300$ & $<300$ \\
\hline $\mathrm{Cr}$ & 20,000 & 20,000 \\
\hline $\mathrm{Cu}$ & 4000 & 5000 \\
\hline$F$ & 18,000 & 50,000 \\
\hline $\mathrm{Fe}$ & 50,000 & 60,000 \\
\hline $\mathrm{Ga}$ & 500 & 700 \\
\hline $\mathrm{Ge}$ & $<100$ & $<100$ \\
\hline $\mathrm{H}$ & 2800 & 4300 \\
\hline Hf & $<300$ & $<300$ \\
\hline $\mathrm{K}$ & 5000 & 5000 \\
\hline $\mathbf{L i}$ & $<100$ & $<100$ \\
\hline $\mathrm{Mg}$ & 10,000 & 15,000 \\
\hline $\mathrm{Mn}$ & 5000 & 3000 \\
\hline Mo & 3000 & 4000 \\
\hline $\mathrm{Na}$ & 1000 & 1000 \\
\hline $\mathrm{Nb}$ & $<300$ & $<300$ \\
\hline $\mathrm{Ni}$ & 20,000 & 20,000 \\
\hline$P$ & $<3000$ & $<3000$ \\
\hline $\mathrm{Pb}$ & 3000 & 2000 \\
\hline $\mathrm{Pu}$ & 48,900 & 89,800 \\
\hline $\mathrm{Si}$ & 100,000 & 100,000 \\
\hline $\mathrm{Sn}$ & $<300$ & $<300$ \\
\hline $\mathrm{Sr}$ & $<100$ & 300 \\
\hline $\mathrm{Ta}$ & $<3000$ & $<3000$ \\
\hline $\mathrm{Ti}$ & 40,000 & 40,000 \\
\hline $\mathrm{TI}$ & $<300$ & $<300$ \\
\hline V & $<100$ & $<100$ \\
\hline$W$ & $<3000$ & $<3000$ \\
\hline$Y$ & 100 & 100 \\
\hline $\mathrm{Zn}$ & 6000 & 6000 \\
\hline $\mathrm{Zr}$ & 5000 & 5000 \\
\hline
\end{tabular}

a Ash sample used was BARFIRC. 
TABLE 1.14 Elemental Composition of Filter Residue and Dissolver Heel Obtained during Small-Scale Cascade Dissolution Studies of Calcined Rocky Flats Ash in $\mathrm{HNO}_{3} / \mathrm{HF}$; Experiment $5^{\mathrm{a}}$

\begin{tabular}{|c|c|c|}
\hline \multirow[b]{2}{*}{ Element } & \multicolumn{2}{|c|}{ Composition, $\mathrm{mg} / \mathrm{kg}$} \\
\hline & Filter Residue & Dissolver Heel \\
\hline $\mathrm{Ag}$ & 200 & 100 \\
\hline $\mathrm{Al}$ & 30,000 & 20,000 \\
\hline B & 700 & 500 \\
\hline $\mathrm{Ba}$ & 4000 & 2000 \\
\hline $\mathrm{Be}$ & 200 & 200 \\
\hline $\mathrm{Bi}$ & $<100$ & $<100$ \\
\hline $\mathrm{C}$ & 7100 & $\cdots$ \\
\hline $\mathrm{Ca}$ & 20,000 & 15,000 \\
\hline $\mathrm{Cd}$ & 200 & 100 \\
\hline $\mathrm{Ce}$ & $<500$ & $<500$ \\
\hline $\mathrm{Cl}$ & 700 & 1000 \\
\hline Co & $<100$ & $<100$ \\
\hline $\mathrm{Cr}$ & 15,000 & 10,000 \\
\hline $\mathrm{Cu}$ & 3000 & 2000 \\
\hline $\mathbf{F}$ & 16,000 & 15,000 \\
\hline $\mathrm{Fe}$ & 80,000 & 60,000 \\
\hline $\mathrm{Ga}$ & 200 & $<100$ \\
\hline $\mathrm{Ge}$ & $<100$ & $<100$ \\
\hline $\mathrm{H}$ & 14,000 & $\ldots$ \\
\hline Hf & $<300$ & $<300$ \\
\hline K & 7000 & 5000 \\
\hline $\mathrm{Li}$ & $<100$ & $<100$ \\
\hline $\mathrm{Mg}$ & 4000 & 3000 \\
\hline $\mathrm{Mn}$ & 3000 & 1500 \\
\hline Mo & 1000 & 600 \\
\hline $\mathrm{Na}$ & 2500 & 2000 \\
\hline $\mathrm{Nb}$ & $<300$ & $<300$ \\
\hline $\mathrm{Ni}$ & 10,000 & 5000 \\
\hline $\mathbf{P}$ & $<3000$ & $<3000$ \\
\hline $\mathrm{Pb}$ & 4000 & 3000 \\
\hline $\mathrm{Pu}$ & {$[4,100$} & 8430 \\
\hline $\mathrm{Si}$ & 10,000 & 80,000 \\
\hline Sn & 1500 & 1000 \\
\hline $\mathrm{Sr}$ & 300 & 200 \\
\hline $\mathrm{Ta}$ & $<3000$ & $<3000$ \\
\hline $\mathrm{Ti}$ & 50,000 & 30,000 \\
\hline $\mathrm{T} 1$ & $<300$ & $<300$ \\
\hline V & $<100$ & $<100$ \\
\hline W & $<3000$ & $<3000$ \\
\hline$Y$ & $<100$ & $<100$ \\
\hline $\mathrm{Zn}$ & 5000 & 3000 \\
\hline $\mathrm{Zr}$ & 2000 & 1000 \\
\hline
\end{tabular}

a Ash sample used was BARF2RC.
TABLE 1.15 Elemental Composition of Filter Residue and Dissolver Heel Obtained during Small-Scale Cascade Dissolution Studies of Calcined Rocky Flats Ash in $\mathrm{HNO}_{3} / \mathrm{HF}$; Experiment $6^{\mathrm{a}}$

\begin{tabular}{|c|c|c|}
\hline \multirow[b]{2}{*}{ Element } & \multicolumn{2}{|c|}{ Composition, $\mathrm{mg} / \mathrm{kg}$} \\
\hline & Filter Residue & Dissolver Heel \\
\hline $\mathrm{Ag}$ & 300 & 100 \\
\hline Al & 40,000 & 40,000 \\
\hline $\mathrm{B}$ & 500 & 400 \\
\hline $\mathrm{Ba}$ & 5000 & 5000 \\
\hline $\mathrm{Be}$ & 500 & 400 \\
\hline $\mathrm{Bi}$ & $<100$ & $<100$ \\
\hline $\mathrm{C}$ & 7400 & 8600 \\
\hline $\mathrm{Ca}$ & 15,000 & 20,000 \\
\hline $\mathrm{Cd}$ & 400 & 400 \\
\hline $\mathrm{Ce}$ & $<500$ & $<500$ \\
\hline $\mathrm{Cl}$ & 800 & 1100 \\
\hline Co & $<300$ & $<300$ \\
\hline $\mathrm{Cr}$ & 10,000 & 10,000 \\
\hline $\mathrm{Cu}$ & 3000 & 3000 \\
\hline$F$ & 17,000 & 38,000 \\
\hline $\mathrm{Fe}$ & 40,000 & 40,000 \\
\hline $\mathrm{Ga}$ & $<100$ & $<100$ \\
\hline $\mathrm{Ge}$ & $<100$ & $<100$ \\
\hline $\mathrm{H}$ & 16,000 & 6700 \\
\hline Hf & $<300$ & $<300$ \\
\hline $\mathrm{K}$ & 10,000 & 10,000 \\
\hline $\mathrm{Li}$ & $<100$ & $<100$ \\
\hline $\mathrm{Mg}$ & 3000 & 4000 \\
\hline $\mathbf{M n}$ & 2000 & 2000 \\
\hline Mo & 800 & 800 \\
\hline $\mathrm{Na}$ & 5000 & 5000 \\
\hline $\mathrm{Nb}$ & $<300$ & $<300$ \\
\hline $\mathrm{Ni}$ & 5000 & 5000 \\
\hline $\mathbf{P}$ & $<1000$ & $<1000$ \\
\hline $\mathrm{Pb}$ & 5000 & 5000 \\
\hline $\mathrm{Pu}$ & 16,600 & 16,800 \\
\hline $\mathrm{Si}$ & 100,000 & 80,000 \\
\hline $\mathrm{Sn}$ & $<300$ & $<300$ \\
\hline $\mathrm{Sr}$ & 500 & 400 \\
\hline $\mathrm{Ta}$ & $<3000$ & $<3000$ \\
\hline $\mathrm{Ti}$ & 40,000 & 40,000 \\
\hline $\mathrm{Tl}$ & $<300$ & $<300$ \\
\hline V & $<100$ & $<100$ \\
\hline$W$ & $<3000$ & $<3000$ \\
\hline $\mathrm{Y}$ & 300 & 300 \\
\hline $\mathrm{Zn}$ & 5000 & 5000 \\
\hline $\mathrm{Zr}$ & 1500 & 300 \\
\hline
\end{tabular}

a Ash sample used was BARF2RC. 
TABLE 1.16 Elemental

Composition of Los Alamos

Incinerator Ash (RGBASH1) after

Fluorination at $250^{\circ} \mathrm{C}$ with $\mathrm{F}_{2}$;

First Batch $^{\mathbf{a}}$

\begin{tabular}{lcc}
\hline & \multicolumn{2}{c}{ Composition $^{\mathrm{b}}$} \\
\cline { 2 - 3 } Element & $\begin{array}{c}\text { Fine Size } \\
\text { Fraction }\end{array}$ & $\begin{array}{c}\text { Coarse Size } \\
\text { Fraction }\end{array}$ \\
\hline $\mathrm{Cl}$ & 500 & 1000 \\
$\mathrm{P}$ & $3.8 \%$ & $2.2 \%$ \\
$\mathrm{~S}$ & 155 & 1300 \\
$\mathrm{~F}$ & $40.2 \%$ & 38.0 \\
$\mathrm{C}$ & 860 & 150 \\
$\mathrm{H}$ & $2.0 \%$ & --- \\
$\mathrm{O}$ & --- & 1.8 \\
$\mathrm{Si}$ & $2.0 \%$ & 2.0 \\
$\mathrm{~K}$ & $40.0 \%$ & --- \\
$\mathrm{Fe}$ & $15.0 \%$ & $\ldots-$ \\
$\mathrm{Ca}$ & $8.0 \%$ & --- \\
$\mathrm{Mg}$ & $10.0 \%$ & --- \\
$\mathrm{Ni}$ & $2.0 \%$ & -- \\
$\mathrm{A} 1$ & $2.0 \%$ & --- \\
\hline
\end{tabular}

a Material was sieved, and two finest fractions and two coarsest fractions were separately combined and analyzed.

b Composition is in $\mathrm{mg} / \mathrm{kg}$ unless otherwise indicated.
TABLE 1.17 Elemental Composition of Los Alamos Incinerator Ash (RGBASH1) after Fluorination at $250^{\circ} \mathrm{C}$ with $\mathrm{F}_{2}$; Second Batch

\begin{tabular}{lc}
\hline Element & Composition $^{\mathrm{a}}$ \\
\hline $\mathrm{Cl}$ & 0.170 \\
$\mathrm{P}$ & -- \\
$\mathrm{S}$ & 745 \\
$\mathrm{~F}$ & $34.0 \%$ \\
$\mathrm{C}$ & 670 \\
$\mathrm{H}$ & 2500 \\
$\mathrm{O}$ & $3.9 \%$ \\
$\mathrm{Si}$ & $4.0 \%$ \\
$\mathrm{~K}$ & $10.0 \%$ \\
$\mathrm{Fe}$ & $5.0 \%$ \\
$\mathrm{Ca}$ & $2.0 \%$ \\
$\mathrm{Na}$ & $5.0 \%$ \\
$\mathrm{Mg}$ & $2.0 \%$ \\
$\mathrm{Ni}$ & $2.0 \%$ \\
$\mathrm{Al}$ & $2.0 \%$ \\
\hline
\end{tabular}

a Composition is in $\mathrm{mg} / \mathrm{kg}$ unless otherwise indicated. 
Figure 1.1 shows IR absorption peaks and the vapor species believed to produce the peaks during fluorination of a Los Alamos ash sample at $250^{\circ} \mathrm{C}$ with $\mathrm{F}_{2}$. The only species that is not evident is $\mathrm{ClF}_{3}$, because it is an excellent fluorinating agent, being reduced to $\mathrm{CIF}$ and then to $\mathrm{Cl}_{2}$. The spectrum in Fig. 1.1 shows that the aliquot of $\mathrm{F}_{2}$ used to fluorinate the ash is depleted, because no $\mathrm{ClF}_{3}$ is observed to be present, and $\mathrm{POF}_{3}$ would be converted to $\mathrm{PF}_{5}$.

Figures 1.2 and 1.3 are FTIR spectra taken approximately $20 \mathrm{~min}$ apart during ash fluorination with $\mathrm{F}_{2}$. The spectra show the rapid appearance and disappearance of $\mathrm{ClF}_{3}$ along with significant CIF. In Fig. 1.2, $\mathrm{ClF}_{3}$ is visible as the envelope of bands centered around $701 \mathrm{~cm}^{-1}$. Note in Fig. 1.3 that $\mathrm{ClF}_{3}$ is totally gone with $\mathrm{Cl}_{2}$ in excess; $\mathrm{ClF}$ will persist a while longer, but it too will disappear. The forward progress of the fluorination reaction can be observed by noting the increase in absorbance values for the more strongly absorbing species (e.g., $\mathrm{PF}_{5}$ or $\mathrm{COCl}_{2}$ ).

Figures 1.4 and 1.5 were taken at the beginning and at the end, respectively, of a batch fluorination before the sample was removed from the furnace and reground. Figure 1.4 shows a rare occurrence of $\mathrm{ClF}_{3}$ and $\mathrm{ClF}$ together before they are removed by fluorinating the ash matrix. Figure 1.4 also shows other vapor species, mainly $\mathrm{POF}_{3}$. As the fluorine-depleted mixture is repeatedly discarded and fresh $\mathrm{F}_{2}$ injected, the spectrum changes to the one in Fig. 1.5, in which $\mathrm{PF}_{5}$, $\mathrm{SiF}_{4}$, and $\mathrm{CO}_{2}$ are essentially gone. (Note the difference in absorbance scale between Fig. .4 and Fig. 1.5.) Only those species that are difficult to fluorinate or are in great abundance remain, as shown in Fig. 1.5. In this case, sulfate is still yielding $\mathrm{SO}_{2} \mathrm{~F}_{2}$. The very stable species $\mathrm{CF}_{4}$ is always present, and the fact that $\mathrm{ClF}_{3}$ and $\mathrm{ClF}$ are present shows that there is an excess of $\mathrm{F}_{2}$ that much chloride remains to be fluorinated.

The following general equations summarize the chemistry associated with the formation of the major species observed in the FTIR spectra:

$$
\begin{gathered}
\mathrm{CO}_{3}{ }^{2-}+\mathrm{F}_{2}--->2 \mathrm{~F}^{-}+\mathrm{CO}_{2}+0.5 \mathrm{O}_{2} \\
\mathrm{SO}_{4}{ }^{2-}+2 \mathrm{~F}_{2}--->2 \mathrm{~F}^{-}+\mathrm{SO}_{2} \mathrm{~F}_{2}+\mathrm{O}_{2} \\
\mathrm{PO}_{4}{ }^{3-}+3 \mathrm{~F}_{2}-->\mathrm{POF}_{3}+1.5 \mathrm{O}_{2}+3 \mathrm{~F}^{-} \\
\mathrm{POF}_{3}+\mathrm{F}_{2}--->\mathrm{PF}_{5}+0.5 \mathrm{O}_{2} \\
\mathrm{SiO}_{2}+2 \mathrm{~F}_{2}-->\mathrm{SiF}_{4}+\mathrm{O}_{2}
\end{gathered}
$$




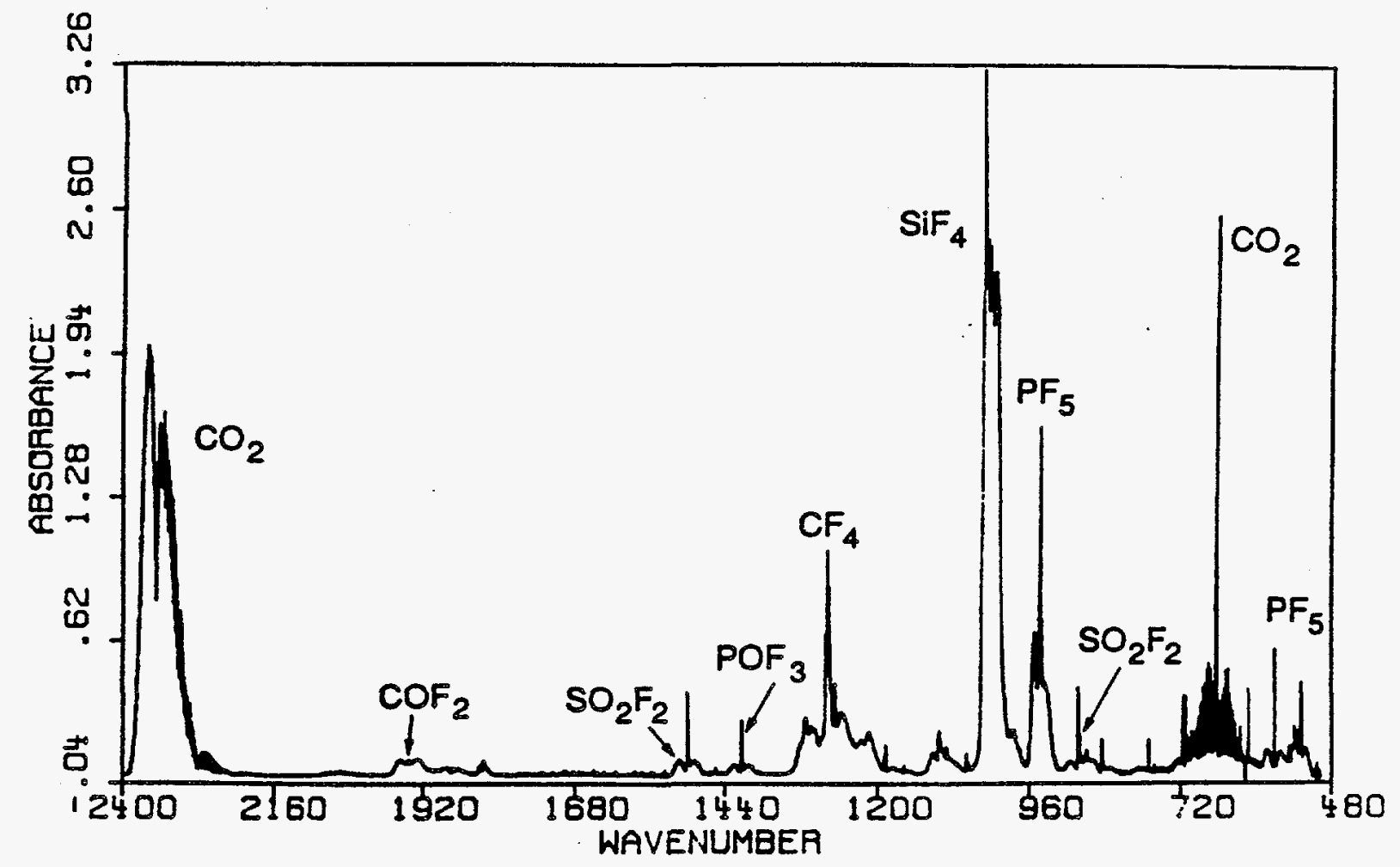

FIGURE 1.1 FTIR Spectrum Showing Absorbances for Major Vapor Species Produced during Fluorination of Los Alamos Incinerator Ash (Sample RGBASH1) at $250^{\circ} \mathrm{C}$ Using $\mathrm{F}_{2}$ 


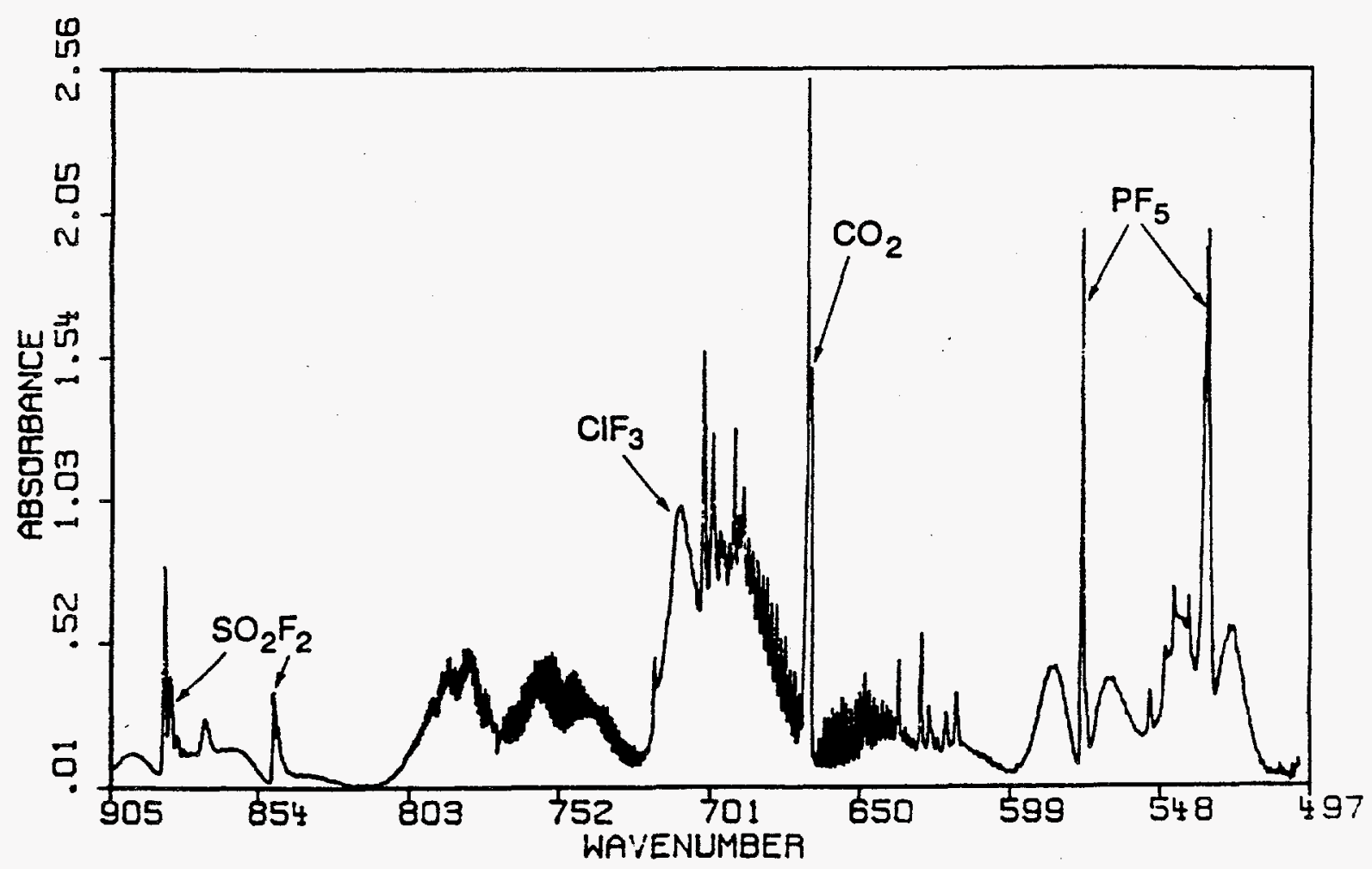

FIGURE 1.2 FTIR Spectrum Showing the Brief Presence of the Superfluorinating Agents CIF $_{3}$ and CIF

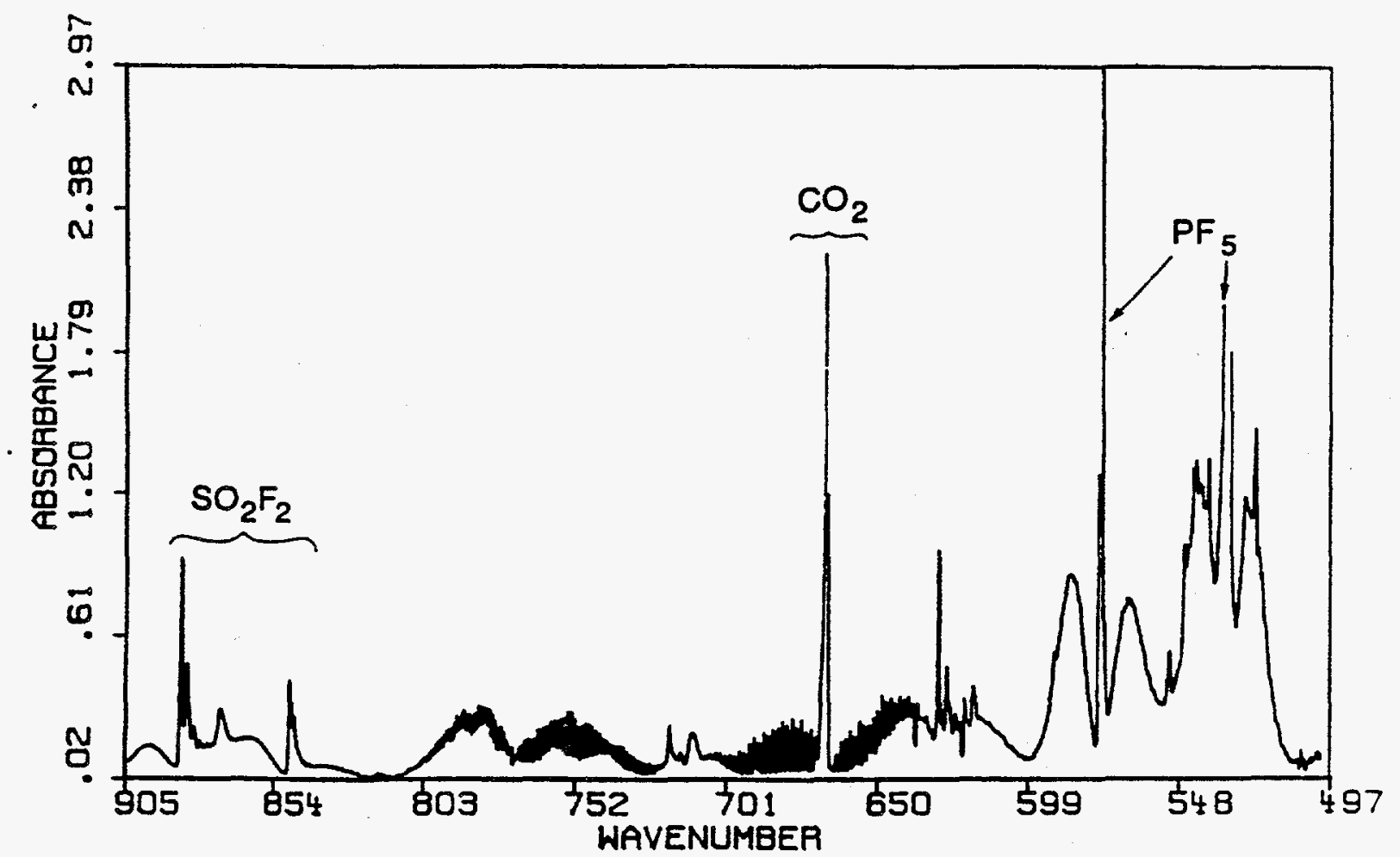

FIGURE 1.3 FTIR Spectrum Obtained 20 min after Spectrum Shown in Fig. 1.2. Peaks associated with $\mathrm{CIF}_{3}$ and $\mathrm{CIF}$ have disappeared. 


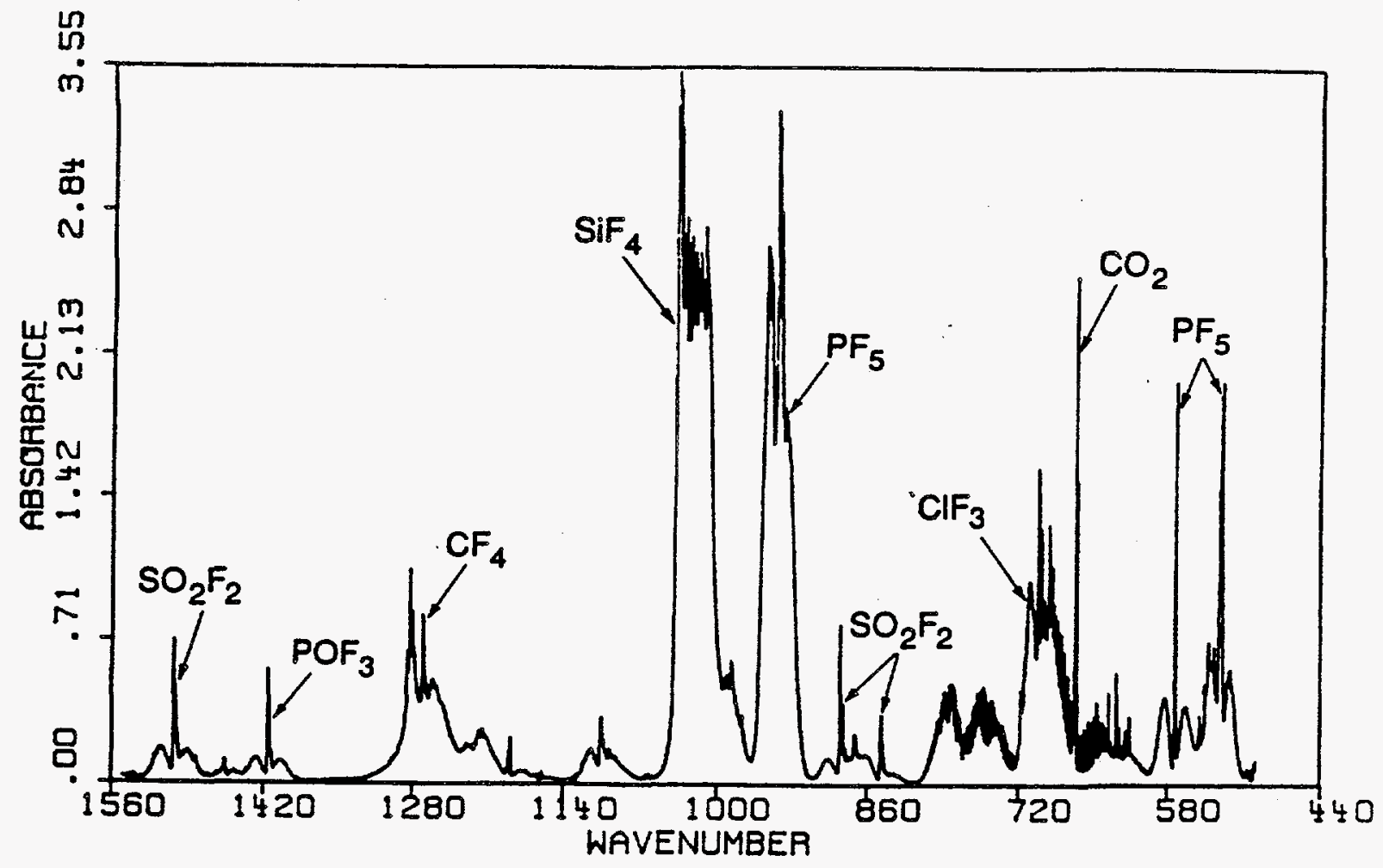

FIGURE 1.4 FTIR Spectrum Taken at the Beginning of Fluorination of Los Alamos Ash at $250^{\circ} \mathrm{C}$. Compare this spectrum with that in Figure 1.5, taken at the end of fluorination.

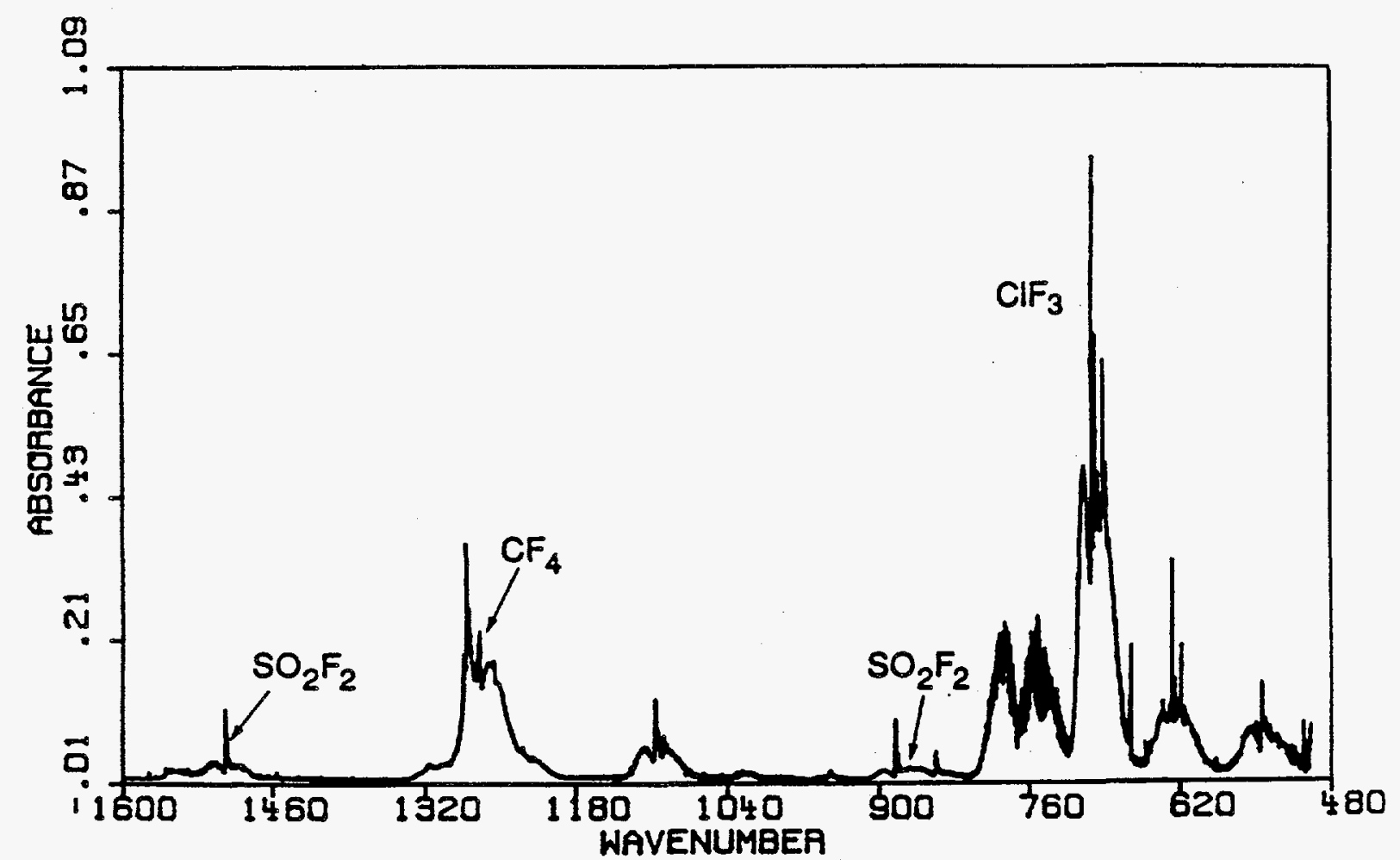

FIGURE 1.5 FTIR Spectrum Taken at the End of Fluorination of Los Alamos Ash at $250^{\circ} \mathrm{C}$ Using $\mathbf{F}_{2}$ 
The species $\mathrm{ClF}_{3}$ is produced by fluorination of any chloride. For example, if $\mathrm{KCl}$ is fluorinated in an initial excess of $\mathrm{F}_{2}$, we get $\mathrm{ClF}_{3}$ only. However, if $\mathrm{ClF}_{3}$ goes on to fluorinate the ash, we will eventually get more $\mathrm{ClF}$ and then $\mathrm{Cl}_{2}$. By adding $\mathrm{F}_{2}$ directly to this gas mixture, we may remake $\mathrm{ClF}_{3}$. The attainment of equilibrium is greatly enhanced by flowing the gas through the furnaces, which are at $250^{\circ} \mathrm{C}$. Therefore, we have

$$
\begin{aligned}
& 250^{\circ} \mathrm{C} \\
& \mathrm{KCl}+\text { excess } \mathrm{F}_{2} \text {---------> } \mathrm{KF}+\mathrm{ClF}_{3} \\
& 2 \mathrm{ClF}_{3}+\text { ash matrix }-->2 \mathrm{ClF}+4 \mathrm{~F} \text { in ash matrix } \\
& 2 \mathrm{ClF}+\text { ash matrix }--->\mathrm{Cl}_{2}+2 \mathrm{~F} \text { in ash matrix. }
\end{aligned}
$$

The $\mathrm{ClF}$ and $\mathrm{ClF}_{3}$ may be regenerated by the reactions

$$
\begin{aligned}
& 250^{\circ} \mathrm{C} \\
& \mathrm{Cl}_{2}+\mathrm{F}_{2} \cdots-\ldots \mathrm{ClF} \\
& 250^{\circ} \mathrm{C} \\
& 2 \mathrm{ClF}+2 \mathrm{~F}_{2}-------->2 \mathrm{ClF}_{3} \text {. }
\end{aligned}
$$

The reason why we can see both $\mathrm{ClF}_{3}$ and $\mathrm{ClF}$ in the experiments is as follows. The species $\mathrm{ClF}$ is stable under pressure and does not disproportionate at room temperature. However, if one condenses equimolar amounts of $\mathrm{F}_{2}$ and $\mathrm{Cl}_{2}$ in a pressure vessel, a reaction does not take place until the species are gaseous and sometimes not until a temperature of $100^{\circ} \mathrm{C}$ is reached. Suddenly, the elements react to give $\mathrm{ClF}_{3}$ and $\mathrm{Cl}_{2}$ with a trace of $\mathrm{ClF}$. To get $\mathrm{ClF}$, we raise the temperature of the mixture to approximately $250^{\circ} \mathrm{C}$. Then $\mathrm{ClF}_{3}$ and $\mathrm{Cl}_{2}$ give $\mathrm{ClF}$ in a good percentage.

Another batch of ash RGBASH1 (180.0 g) was treated with 550 torr of $\mathrm{F}_{2}$ at $500^{\circ} \mathrm{C}$, producing a final weight of $161.3 \mathrm{~g}$. The sample had about 15.1 wt\% plutonium. Again, by FTIR spectroscopy, $3.64 \mathrm{~g}(2.02 \mathrm{wt} \%)$ of $\mathrm{C}$ was volatilized as $\mathrm{CO}_{2}$ and $\mathrm{CF}_{4} ; 2.74 \mathrm{~g}(1.51 \mathrm{wt} \%)$ of $\mathrm{Si}$ was volatilized as $\mathrm{SiF}_{4}$; $2.24 \mathrm{~g}(1.25 \mathrm{wt} \%) \mathrm{S}$ was lost as $\mathrm{SO}_{2} \mathrm{~F}_{2}$, and $7.41 \mathrm{~g}$ (4.12 wt\%) of $\mathrm{P}$ was lost as $\mathrm{PF}_{5}$ and $\mathrm{POF}_{3}$. Table 1.18 summarizes the analytical results for the
TABLE 1.18 Elemental Composition of Los Alamos Incinerator Ash (RGBASH1) after Fluorination at $500^{\circ} \mathrm{C}$ with $\mathbf{F}_{2}$

\begin{tabular}{lc} 
Element & Composition, Wt\% \\
\hline $\mathrm{Cl}$ & 0.100 \\
$\mathrm{P}$ & 1.40 \\
$\mathrm{~S}$ & 935 \\
$\mathrm{~F}$ & 36.0 \\
$\mathrm{C}$ & $250^{\mathrm{a}}$ \\
$\mathrm{H}$ & $1600^{\mathrm{a}}$ \\
$\mathrm{O}$ & 1.8 \\
$\mathrm{Si}$ & $5000^{\mathrm{a}}$ \\
$\mathrm{K}$ & 20.0 \\
$\mathrm{Fe}$ & 5.0 \\
$\mathrm{Ca}$ & 5.0 \\
$\mathrm{Na}$ & 5.0 \\
$\mathrm{Mg}$ & 4.0 \\
$\mathrm{Ni}$ & 3.0 \\
$\mathrm{Al}$ & 2.0 \\
\hline
\end{tabular}

a Composition is in $\mathrm{mg} / \mathrm{kg}$. 
ash after fluorination at $500^{\circ} \mathrm{C}$. During all of these experiments, there was no evidence of volatilization of $\mathrm{Pu}$ as $\mathrm{PuF}_{6}(\mathrm{~g})$.

To study any differences in chemistry and dissolution parameters, ash that was prefluorinated with $\mathrm{F}_{2}$ at $250^{\circ} \mathrm{C}$ was dissolved in minicascade dissolvers, and the ash heel and filter residue were analyzed. Results of the chemical analyses are presented in Table 1.19 for comparison with analyses for ash heel and filter residue of calcined Rocky Flats ash dissolved in $\mathrm{HNO}_{3} / \mathrm{HF}$ (Tables 1.10-1.15).

\subsubsection{X-ray Powder Patterns}

A sample of Los Alamos virgin ash, a sample of fine-mesh Los Alamos ash prefluorinated with $\mathrm{F}_{2}$ at $250^{\circ} \mathrm{C}$, and another Los Alamos ash sample prefluorinated at $500^{\circ} \mathrm{C}$ with $\mathrm{F}_{2}$ were examined using the Debye-Scherrer technique. The $\mathrm{X}$-ray scattering from the virgin ash was overwhelmingly dominated by $\mathrm{PuO}_{2}$ of moderate crystallinity. The only diffraction lines not due to $\mathrm{PuO}_{2}$ were attributable to a trace amount of $\mathrm{KCl}$.

The X-ray patterns from the two prefluorinated samples were similar to that observed for the virgin ash but had a more complex structure. The $\mathrm{PuO}_{2}$ diffraction lines were extremely weak for these samples. Many of the diffraction lines in the prefluorinated samples could not be identified. Table 1.20 summarizes condensed phases that were specifically looked for, but could not be correlated with any of the diffraction lines. Unfortunately, X-ray diffraction patterns for many possible ternary fluorides of plutonium have never been reported in the literature.
TABLE 1.19 Elemental Composition of Filter Residue and Dissolver Heel Obtained during Small-Scale Cascade Dissolution Studies of Prefluorinated Virgin Los Alamos Ash in $\mathrm{HNO}_{3}{ }^{\mathrm{a}}$

\begin{tabular}{|c|c|c|}
\hline \multirow[b]{2}{*}{ Element } & \multicolumn{2}{|c|}{ Composition, $\mathrm{mg} / \mathrm{kg}$} \\
\hline & Filter Residue & Dissolver Heel \\
\hline $\mathrm{Ag}$ & 100 & 100 \\
\hline $\mathrm{Al}$ & 20,000 & 20,000 \\
\hline B & 15,000 & 7,000 \\
\hline $\mathrm{Ba}$ & 600 & 500 \\
\hline $\mathrm{Be}$ & $<10$ & $<10$ \\
\hline $\mathrm{Bi}$ & $<100$ & $<100$ \\
\hline $\mathrm{C}$ & 12,000 & 10,000 \\
\hline $\mathrm{Ca}$ & 50,000 & 60,000 \\
\hline $\mathrm{Cd}$ & 2000 & 2,000 \\
\hline $\mathrm{Ce}$ & $<500$ & $<500$ \\
\hline $\mathrm{Cl}$ & 700 & 700 \\
\hline Co & $<100$ & $<100$ \\
\hline $\mathrm{Cr}$ & 20,000 & 15,000 \\
\hline $\mathrm{Cu}$ & 2000 & 1,500 \\
\hline $\mathrm{F}$ & 16,000 & 120,000 \\
\hline $\mathrm{Fe}$ & 60,000 & 50,000 \\
\hline $\mathrm{Ga}$ & 4,000 & 3,000 \\
\hline $\mathrm{Ge}$ & $<100$ & $<100$ \\
\hline $\mathrm{H}$ & 2,700 & $\ldots$ \\
\hline $\mathrm{Hf}$ & $<500$ & $<500$ \\
\hline K & 10,000 & 100,000 \\
\hline $\mathrm{Li}$ & 100 & 100 \\
\hline $\mathrm{Mg}$ & 30,000 & 30,000 \\
\hline $\mathrm{Mn}$ & 2,000 & 1.000 \\
\hline Mo & 2,000 & 300 \\
\hline $\mathrm{Na}$ & 40,000 & 30,000 \\
\hline $\mathrm{Nb}$ & $<300$ & $<300$ \\
\hline $\mathrm{Ni}$ & 8,000 & 5,000 \\
\hline $\mathrm{O}$ & 118,000 & 90,000 \\
\hline$P$ & 18,000 & 17,000 \\
\hline $\mathrm{Pb}$ & $<100$ & $<100$ \\
\hline $\mathrm{Pu}$ & 81,000 & 157,000 \\
\hline$S$ & 12,000 & 12,000 \\
\hline $\mathrm{Si}$ & 50,000 & 50,000 \\
\hline Sn & $<300$ & $<300$ \\
\hline $\mathrm{Sr}$ & 1,500 & 2,000 \\
\hline $\mathrm{Ta}$ & $<3,000$ & $<3,000$ \\
\hline $\mathrm{Ti}$ & 7,000 & 6,000 \\
\hline $\mathrm{Tl}$ & $<300$ & $<300$ \\
\hline V & $<100$ & $<100$ \\
\hline W & $<3,000$ & $<3,000$ \\
\hline$Y$ & 1,500 & 2,000 \\
\hline $\mathrm{Zn}$ & 2,000 & 300 \\
\hline $\mathrm{Zr}$ & 300 & 300 \\
\hline
\end{tabular}


TABLE 1.20 Summary of X-ray Diffraction Results on Virgin and

Fluorinated Los Alamos Ash (BASH)

\author{
Virgin Ash \\ Scattering dominated by $\mathrm{PuO}_{2}$ \\ Trace $\mathrm{KCl}$ (and possibly $\mathrm{MgO}$ ) \\ Phases not observed: \\ Binary oxides of $\mathrm{K}, \mathrm{Ca}, \mathrm{Fe}$, and $\mathrm{Si}$ \\ Carbonates of $\mathrm{Na}, \mathrm{K}, \mathrm{Ca}$, and $\mathrm{Mg}$ \\ Phosphates of $\mathrm{Pu}, \mathrm{Ca}$, and $\mathrm{K}$
}

Fluorinated Ash

Small amount of scattering due to $\mathrm{PuO}_{2}$

Phases not observed:

Binary fluorides of $\mathrm{Na}, \mathrm{K}, \mathrm{Fe}, \mathrm{Ca}$, and $\mathrm{Pu}$

Double fluorides including $\mathrm{CaPuF}_{6}, \mathrm{~K}_{2} \mathrm{PuF}_{2}, \mathrm{~K}_{3} \mathrm{PuF}_{7}$, and $\mathrm{KPu}(\mathrm{V})$ fluorides

Oxyfluorides including $\mathrm{PuOF}_{4}$ and $\mathrm{PuO}_{2} \mathrm{~F}_{2}$

Fluorosilicates including $\mathrm{K}_{2} \mathrm{SiF}_{6}, \mathrm{~K}_{3} \mathrm{SiF}_{7}$, and $\mathrm{CaSiF}_{6}$.

\title{
1.3.2.3 Infrared Spectra
}

Infrared spectra were run on Nujol and fluorocarbon mulls for the virgin ash and two prefluorinated samples discussed in the previous section (Fig. 1.6). The principal features in the spectrum of the virgin ash are a broad, featureless absorption peak near $1400 \mathrm{~cm}^{-1}$, a region where phosphate, carbonate, and other multiple-bonded oxyanions have strong absorptions, and strong metal-oxygen absorption below $600 \mathrm{~cm}^{-1}$. The spectra of the two fluorinated samples are essentially identical and show the ingrowth of a strong absorption near $900 \mathrm{~cm}^{-1}$ [plutonyl(VI) stretching region]. Indeed a sample of $\mathrm{K}_{\mathrm{x}} \mathrm{UO}_{2} \mathrm{~F}_{2+\mathrm{x}}$ precipitated from a solution of uranyl ion with excess fluoride has an infrared spectrum very similar to that of the fluorinated samples. The infrared spectra are thus inaccurate compared with the $\mathrm{X}$-ray results as a means of indicating effective conversion of the $\mathrm{PuO}_{2}$ to another form, possibly a double plutonyl fluoride, and of other oxides to fluorides.

\subsubsection{Dissolution Behavior}

The virgin ash and two refluorinated ash samples were dissolved in $8 \mathrm{M} \mathrm{HNO}_{3} / 0.1 \mathrm{M}$ $\mathrm{Al}\left(\mathrm{NO}_{3}\right)_{3}$ solution. While the virgin ash was resistant to dissolution, the fluorinated ash samples behaved similarly, in that substantial $(\sim 70 \%)$ dissolution occurred immediately at room temperature to give light green solutions and off-white residues. Brief heating to reflux for less than $1 \mathrm{~h}$ did not affect total dissolution of the residue. 
(A)

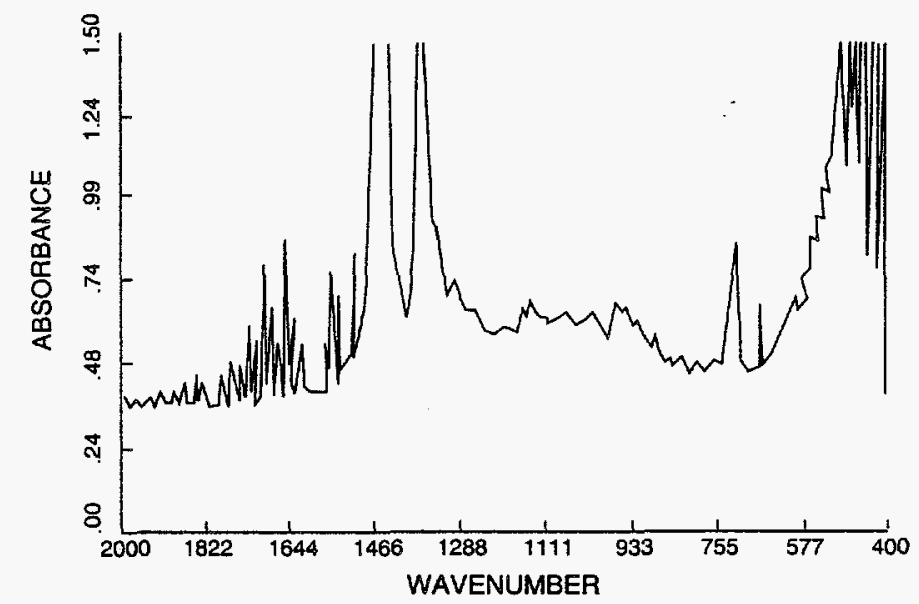

(B)

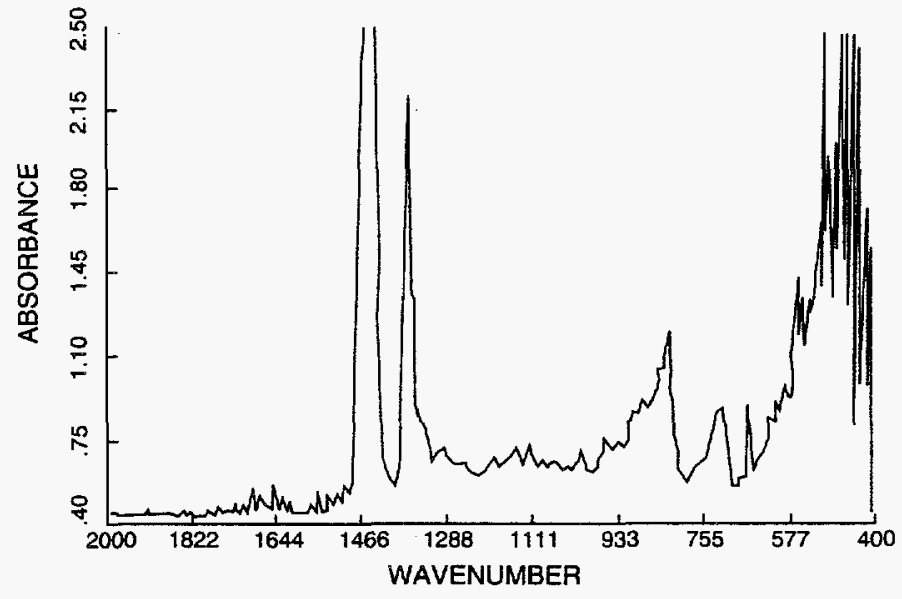

(C)

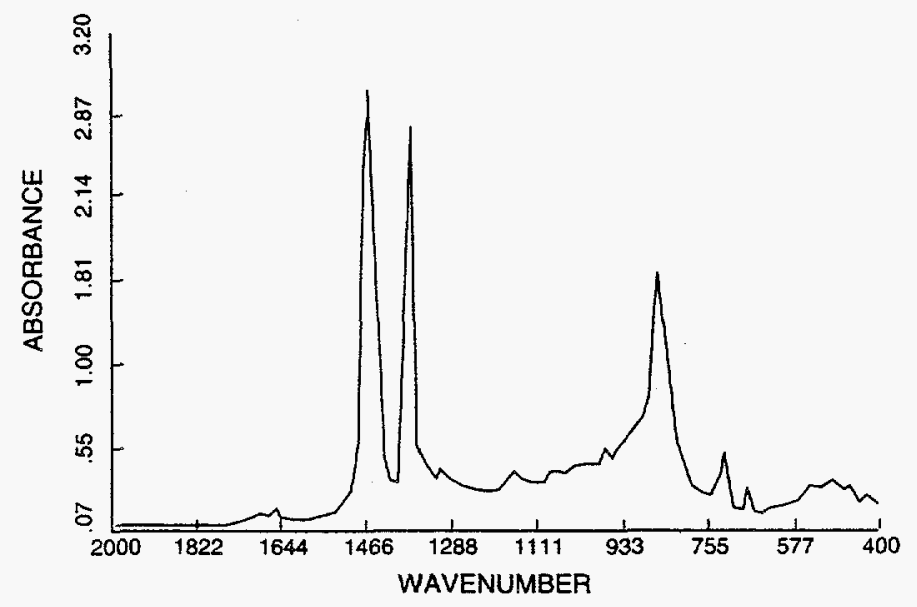

FIGURE 1.6 Infrared Spectra (Nujol mulls) of (a) Virgin Ash, (b) Fluorinated Ash, and (c) $\mathrm{K}_{\mathrm{x}} \mathrm{UO}_{2} \mathrm{~F}_{2+x^{*}}$ Asterisks denote Nujol bands. 


\section{REFERENCES}

\section{BARNEY}

G. S. Barney, "The Kinetics of Plutonium Oxide Dissolution in Nitric/Hydrofluoric Acid Mixtures," J. Inorg. Chem. 39, 1665 (1977)

\section{BUTLER}

F. E. Butler, J. D. Moseley, and G. F. Molen, Recovery of Plutonium from Incinerator Ash, Rocky Flats Plant Report RFP-396 (July 31, 1964).

\section{CHRISTENSEN}

D. C. Christensen, D. F. Bowersox, B. J. McKerley, and R. L. Nance, Wastes from Plutonium Conversion and Scrap Recovery Operations, Los Alamos National Laboratory Report L1-11069-MS (March 1988).

\section{CHRISTENSEN}

E. L. Christensen and W. E. J. Maraman, Plutonium Processing at the Los Alamos Scientific Laboratory, Los Alamos Scientific Laboratory Report LA-3542 (April 1969).

\section{DELEGARD}

C. H. Delegard, Laboratory Tests on Plutonium Recovery from Rocky Flats Ash Using Nitric AcidCalcium Fluoride Leaching, Rockwell Hanford Operations Report SD-CP-DTR-005 (1984).

\section{FOSTER}

R. S. Foster, Incineration of Plutonium Contaminated Waste Materials, Rocky Flats Plant Report RFP-735 (May 9, 1968).

\section{FREED}

E. J. Freed, SRL Incinerator Components Test Facility, E. I. duPont de Nemours \& Co., Savannah River Laboratory Report DP-1940 (August 1982).

\section{JOHNSON}

T. C. Johnson, Recovery of Plutonium from Incinerator Ash at Rocky Flats, Rocky Flats Plant Report RFP-2520 (December 21, 1976).

\section{KAZANJIAN}

A. R. Kazanjian and J. R. Stevens, Dissolution of Plutonium Oxide in Nitric Acid at High Hydrofluoric Acid Concentrations, Rockwell International, Rocky Flats Plant Report RFP-3609 (June 15, 1984). 


\section{MCNEESE}

W. D. McNeese and W. J. Maraman, Incinerator for Radioactive Residues, Los Alamos Scientific Laboratory Report LA-1691 (March 1954).

\section{MCNEESE}

W. D. McNeese and W. J. Maraman, Incinerator for Radioactive Residues, Industrial and Engineering Chemistry 52(2), 79A-81A (1960).

\section{OLSEN}

J. Olsen, "Incinerator," Standard Operating Procedure 422-REC-R05 (February 1989), Group MSTT-12, Los Alamos National Laboratory.

\section{RYAN}

J. L. Ryan and L. A. Bray, "Dissolution of Plutonium Dioxide-A Critical Review," in Actinide Separations, Eds., J. D. Navratil and W. W. Schulz, American Chemical Society, Washington, DC, p. 499 (1980).

\section{TALLENT}

O. K. Tallent and J. C. Mailen, "Study of the Dissolution of Refractory $\mathrm{PuO}_{2}$ in Nitric-Hydrofluroic Acid Dissolvents at $100^{\circ} \mathrm{C}, "$ Nucl. Tech., 32, 167 (1977). 


\section{ACKNOWLEDGMENT}

This work was funded by the U.S. Department of Energy, Office of Nuclear Materials Production. 
This page intentionally left blank. 


\title{
PART 2: ANALYTICAL ELECTRON MICROSCOPY \\ CHARACTERIZATION
}

by

\author{
E. C. Buck, N. L. Dietz, J. K. Bates, E. Van Deventer and D. J. Chaiko
}

\subsection{INTRODUCTION}

Considerable amounts of plutonium-bearing scrap and wastes have been generated over the past 40 years as a result of plutonium processing activities that took place across the defense programs complex [BLUM]. Whether a particular material is classified as a scrap ${ }^{1}$ or a waste depends on whether the plutonium concentration is above or below the economic discard limit (EDL). Calculation of the EDL takes into account the costs associated with processing and waste management and compares these costs with the value of the recovered plutonium [CHRISTENSEN]. If the plutonium content is above the EDL, the material is then scheduled to be reprocessed and is, by definition, a scrap. Waste, on the other hand, is any material that has a plutonium concentration below the EDL and is, therefore, a candidate for disposal. A component of rapidly increasing importance in the EDL calculation is the added cost associated with waste management, which is incurred from any additional processing of the residue or scrap material.

Most scrap plutonium is produced during the fabrication of parts and components from plutonium metal and consists largely of particulate materials. The plutonium-containing residues produced during the chemical processing of that scrap, however, represent a significant portion of the scrap volume. The various residues have been catalogued into two broad categories - low-level scrap and high-level scrap - on the basis of plutonium content, processing costs, and the degree of difficulty associated with reprocessing. A list of these items is given in Table 2.1. The reported plutonium concentrations in the incinerator ash residues range from $<1 \mathrm{wt} \%$ to $>85 \mathrm{wt} \%$ [CHRISTENSEN].

Particularly difficult to process are LECO crucibles. These are ceramic crucibles that are used in analytical procedures, at the Savannah River Site and elsewhere, for the determination of carbon in plutonium dioxide [CHRISTENSEN]. The crucibles contain refractory $\mathrm{PuO}_{2}$ imbedded in the ceramic, along with small amounts of a catalyst that may be copper, iron, or tin.

Current beneficiation strategies for plutonium residues include the incineration of combustibles, such as wood, paper, plastics, and oils; particle size reduction (where necessary); and

1 In the U.S. Department of Energy (DOE) community, the terms scrap and residue are used interchangeably. 
TABLE 2.1 Survey of Plutonium-Containing Scrap ${ }^{a}$

\begin{tabular}{lc}
\hline \multicolumn{1}{c}{ Low-Level Scrap } & High-Level Scrap \\
\hline Graphite & Impure metal \\
Combustibles & Nonspecification metal \\
Incinerator ash & Anode heels \\
Oxide heels & Rich plutonium oxide $(>85 \% \mathrm{Pu})$ \\
Reduction slags & Lean plutonium oxide $(<85 \% \mathrm{Pu})$ \\
Insulation & \\
Ceramics & \\
Scrap metals & \\
Glass & \\
Rubber & \\
Sludge & \\
Chloride-based salts & \\
Pu/U mixtures & \\
Pu/Th mixtures & \\
Pu/Be mixtures & \\
Pu/Np mixtures & \\
Pu/Zr mixtures & \\
Pu/Al mixtures & \\
\hline
\end{tabular}

a Reproduced from [CHRISTENSEN].

washing, leaching, or dissolution with acid. The pregnant solutions are then further processed to yield either a plutonium oxide or $\mathrm{PuF}_{4}$. Metal oxides (both reactive and high-fired) and impure plutonium metal are processed by direct oxide reduction, molten salt extraction, or electrorefining [CHRISTENSEN].

The lack of permanent waste disposal facilities has necessitated temporary storage of plutonium-bearing waste materials at the various production sites. Recently, the hazard levels associated with the temporary storage of plutonium and uranium residues have been questioned because of a number of safety incidents, including drum explosions, at DOE facilities [LOBSENZ]. Radiolytic processes can result in the overpressurizing of those storage drums that contain both organics (plastics) and plutonium compounds, as well as the formation of pyrophoric actinide compounds.

Much of the weapons-grade plutonium waste is marked for immobilization, possibly by vitrification. Current plans call for an immobilization of plutonium-bearing wastes in either a 
borosilicate glass or a Synroc-type ceramic prior to permanent storage. The stable waste form would then be a candidate for disposal in a deep geological repository such as the one proposed at Yucca Mountain in Nevada [GRAY]. Before an optimum waste glass can be formulated, however, the physical and chemical characteristics of the plutonium in the wastes need to be determined. For example, refractory plutonium oxides may require different processing conditions for vitrification compared to plutonium in glass or ceramic phases. Knowledge of the physicochemical nature of the plutonium may also be necessary for accurate performance assessment, should it be decided that the wastes are to be buried in their current form in repositories such as the Waste Isolation Pilot Plant in New Mexico. For instance, incinerator ash may not be a stable waste form and could be susceptible to leaching in the event of contact with groundwater. Zevenbergen investigated the mechanism of municipal ash weathering and found that glassy phases in the ash underwent alteration to secondary phases such as smectite clay and illite, analogous to the corrosion of nuclear waste glasses [ZEVENBERGEN, BATES-1995].

Characterization of radionuclide-contaminated soils or residues involves a number of techniques that provide information at different levels, for example, X-ray diffraction (XRD), sizesieving, wet chemical analysis, and alpha and gamma spectroscopies for bulk characterization. Bulk diffraction techniques can provide representative data, but can miss sub-micron-sized particles and amorphous phases.

In this section, we describe the use of microscopic characterization techniques to determine the physicochemical form of the plutonium in the residues and wastes from DOE facilities, to aid in the development of efficient methods for actinide recovery, and to provide information that could assist in the formulation of waste glasses or ceramics for plutonium immobilization. The basic chemistry and detailed composition of ash and ash heels was reported in Part 1 of this report. The ash was formed in incinerators at temperatures between $200^{\circ} \mathrm{C}$ and $900^{\circ} \mathrm{C}$, although the exact temperature is not known, as it varied depending on what else was placed in the incinerator. The $\mathrm{XRD}$ studies suggested that the plutonium may be present as $\mathrm{PuO}_{2}$. Because the XRD data were complicated by many other reflections from unknown phases, analytical electron microscopy (AEM) was considered to be a good technique for determining the exact nature of plutonium in ash and ash heels. We have used this approach successfully as an aid to soil remediation efforts: by using AEM techniques, we were able to identify the form of uranium in the soil from the Fernald Environmental Management Project [BUCK-1994]. 


\subsection{EXPERIMENTAL PROCEDURE}

\subsubsection{Microscopic Analysis}

Table 2.2 lists the samples that were characterized by AEM in this study. Samples were analyzed by optical microscopy and electron microscopy to provide a detailed picture of the material. The small probe capabilities of AEM and scanning electron microscopy (SEM) were used to determine the structure and composition of the major phases present in the samples, together with those phases containing detectable amounts of plutonium. Comparison of SEM and AEM images from the same sample allows greater confidence in determining whether plutonium-bearing phases seen by AEM are mere peculiarities or are representative of the contamination in the waste sample. However, because the database on plutonium-bearing phases is limited, some phases were observed that had not previously been documented in the literature. In addition, we found that even when plutonium-bearing phases can be identified, little is known about their properties.

\subsubsection{Preparation of Samples for Scanning Electron Microscopy}

In studies for which a representative sample of the bulk material is required, it is necessary to show that one is observing the same regions as the characterization proceeds from use of the optical microscope to the scanning and transmission electron microscopes. Thus, sample preparation requires the use of a resin that is suitable for both optical and scanning electron microscopy as well as ultramicrotomy of the sample for transmission electron microscopy (TEM).

Bulk mineralogy of the samples was investigated by using XRD. Bulk chemical analysis was performed by using emission spectroscopy and radiochemical analysis. Samples were examined in the scanning electron microscope and the transmission electron microscope. For SEM analysis, the sample was mounted in an epoxy resin and polished inside a Blickmann hood.

\subsubsection{Analysis of Samples by Analytical Electron Microscopy}

Analytical electron microscopy is a combination of TEM, energy dispersive spectroscopy (EDS), electron energy loss spectroscopy (EELS), selected area electron diffraction, and convergent beam electron diffraction. High-energy electrons, passing through a solid, interact strongly through a number of inelastic and elastic processes. These processes permit one to obtain useful structural and compositional data on nanometer-sized particles. The point-to-point resolution for images obtained with the transmission electron microscope approaches $0.3 \mathrm{~nm}$, and the smallest region that 
TABLE 2.2 Summary of Results from Sample Surveys

\begin{tabular}{|c|c|}
\hline Sample & Comments and Observations \\
\hline Los Alamos ash & $\begin{array}{l}\text { Plutonium oxide particles found. } \\
\text { Plutonium appeared to surround } \\
\text { some of the particles. Performed } \\
\text { EELS on plutonium particle. }\end{array}$ \\
\hline Rocky Flats ash & $\begin{array}{l}\text { Plutonium oxide particles attached } \\
\text { to silicates. Some evidence of } \\
\text { other forms of plutonium besides } \\
\text { plutonium oxide. }\end{array}$ \\
\hline Rocky Flats ash heel & $\begin{array}{l}\text { Sectioned sample showed plu- } \\
\text { tonium oxide and other plutonium } \\
\text { phases. Aluminum oxide, steel, } \\
\text { zirconium oxide, and various } \\
\text { aluminum silicate particles were } \\
\text { also observed. }\end{array}$ \\
\hline Sand, slag, and crucible & $\begin{array}{l}\text { Plutonium particles were smaller } \\
\text { than in the other samples. A large } \\
\text { amount of zirconium oxide } \\
\text { containing cerium was observed. }\end{array}$ \\
\hline Ground LECO crucible & $\begin{array}{l}\text { Plutonium was found closely } \\
\text { associated with copper. Zirconium } \\
\text { particles were found again. Micro- } \\
\text { tome thin sections showed a } \\
\text { number of plutonium and other } \\
\text { particles. }\end{array}$ \\
\hline
\end{tabular}

can be investigated by using electron diffraction, EDS, and EELS is about $20 \mathrm{~nm}$. The analytical microscopy facility operated by the Nuclear Waste Technology group at Argonne National Laboratory is dedicated to the characterization and analysis of radioactive materials.

Samples for AEM analysis were infiltrated with a small amount of an epoxy resin to form a mold or block. These blocks were thin-sectioned using an ultramicrotome for examination by TEM [BUCK-1995] (see Fig. 2.1a). The ultramicrotome has been specially adapted to handle radioactive materials. The samples were analyzed in a JEOL 2000 FXII transmission electron microscope, operated at $200 \mathrm{keV}$ and equipped with X-ray energy dispersive spectrometers, a Gatan parallel electron energy loss spectrometer, and a Gatan charge-coupled device (CCD) camera for in situ low intensity imaging (see Figure 2.1b). The microscope and analytical instruments are linked to a 

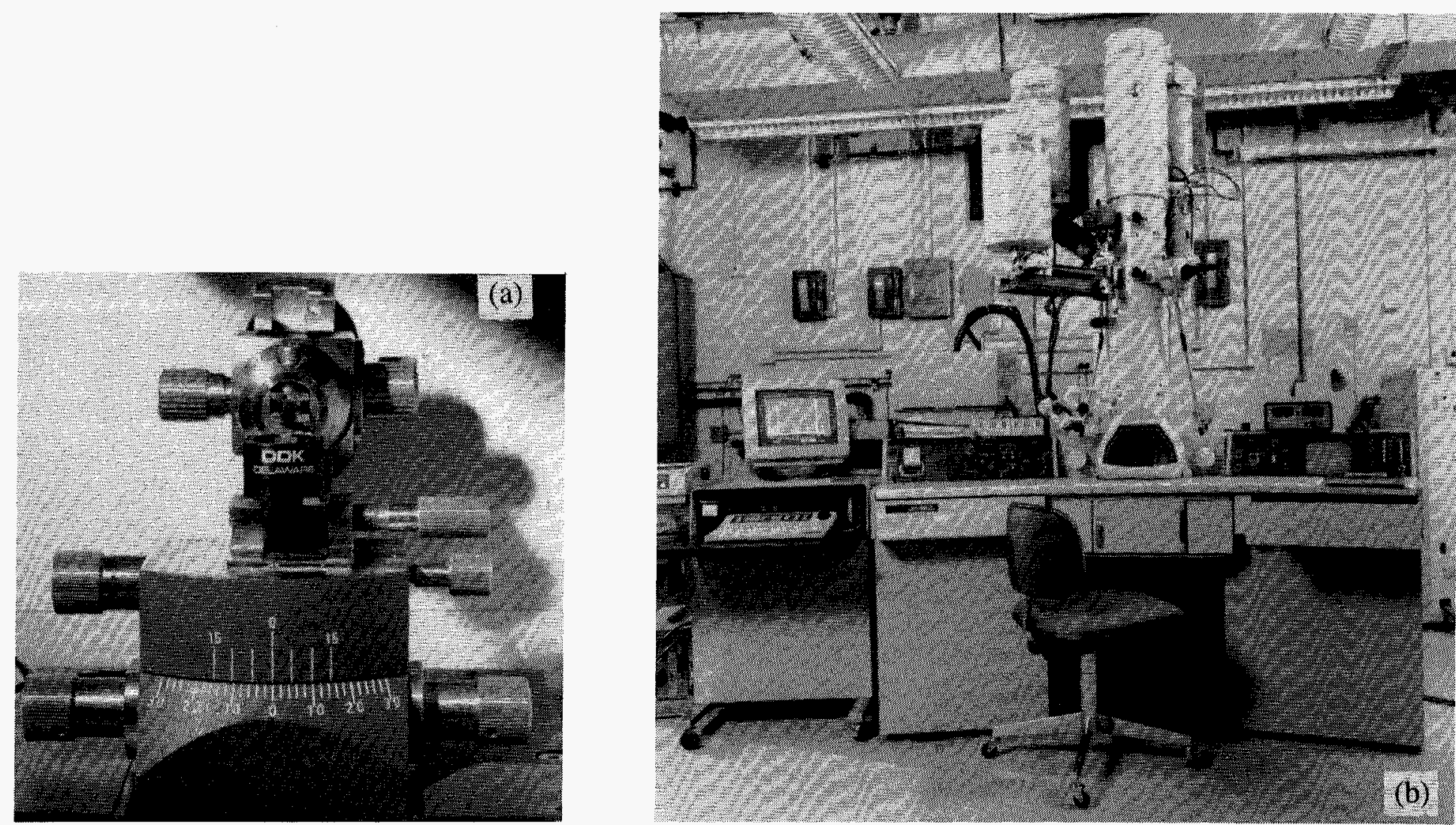

FIGURE 2.1 Analytical Electron Microscopy Facility at Argonne National Laboratory. (a) Photograph of ultramicrotome for preparing TEM thin sections. (b) Photograph of a JEOL 2000 FX II transmission electron microscope. The instrument pictured is equipped with a light-element EDS and Be-window EDS, a Gatan parallel EELS, and Gatan CCD digital imaging system. 
Macintosh 950 Quadra. The microscope and facility are approved for examining radioactive materials. Phases were identified by a combination of EDS, EELS, and electron diffraction.

To determine the d-spacings of phases, electron diffraction patterns were taken while a steady eucentric height and objective lens current were maintained, and also with the brightness control defocused. The microscope camera lengths were determined by using a polycrystalline aluminum standard sample. Quantitative EDS analysis was performed by using the thin-film procedure of Cliff and Lorimer [CLIFF]. Experimentally derived correction factors (k-factors) were obtained from glass and mineral thin-film standards. However, the plutonium k-factor was calculated by extrapolation from uranium and thorium $\mathrm{k}$-factors.

\subsubsection{Particle Size Reduction}

To determine the effect of ultrafine grinding on plutonium liberation, the plutonium samples were ground to an average particle size of $1 \mu \mathrm{m}$ or less by using a McCrone micronizing mill (The McCrone Group, Westmont, $\mathrm{L}$ ). Grinding times of $1-4 \mathrm{~h}$ were sufficient to achieve the desired particle size reduction for a 1-g sample (dry weight) and a top feed size of less than $0.5 \mathrm{~mm}$. The samples were ground in an aqueous slurry with $0.1 \mathrm{wt} \%$ hexametaphosphate as a dispersant. The total sample volume was $4 \mathrm{~mL}$. We used cylindrical zirconia grinding elements from Fisher Scientific. The lack of zirconium-rich phases in the plutonium samples meant that any micron-sized particles shed from the grinding media could easily be distinguished from true sample particles. This would not have been the case with other grinding media, such as agate.

\subsection{RESULTS}

\subsubsection{Plutonium Distribution in the Waste Particle Matrix}

Many of the scrap materials listed in Table 2.1 are multiphasic, heterogeneous materials. Consequently, one might expect to find a heterogeneous distribution of plutonium between discrete particles. In all of the samples that we examined, this was indeed the case. However, we also found evidence of the segregation of plutonium into discrete grains within a larger, nonactinide matrix, as shown in Fig. 2.2. The particle shown is from a sample of Rocky Flats ash heel. The term "heel" refers to the relatively inert material remaining after one or more cycles of leaching/dissolution by mixtures of $\mathrm{HNO}_{3}$ acid and $\mathrm{HF}$. The original feed for this sample, obtained from Lawrence Livermore National Laboratory, was an incinerator ash from the Rocky Flats Plant that was calcined, ground to a particle size of about $10-20 \mu \mathrm{m}$ in a ceramic ball mill, and acid leached. In Fig. 2.2, the plutonium appears as bright, discrete grains, about $1 \mu \mathrm{m}$ in diameter, imbedded in a complex matrix of metal oxides and silica. Figure 2.3 shows the EDS spectrum of the matrix material. In other 


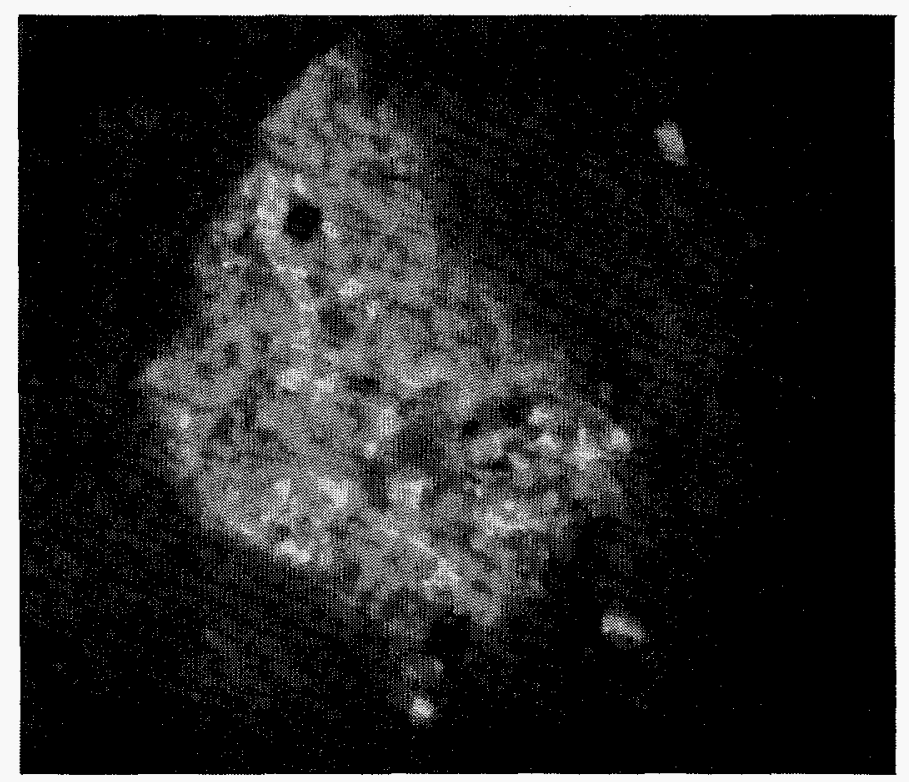

FIGURE 2.2 Photomicrograph of an Ash Heel Particle Showing Plutonium Grains (Bright Spots) in a Matrix of Metal Oxides and $\mathrm{SiO}_{2}$

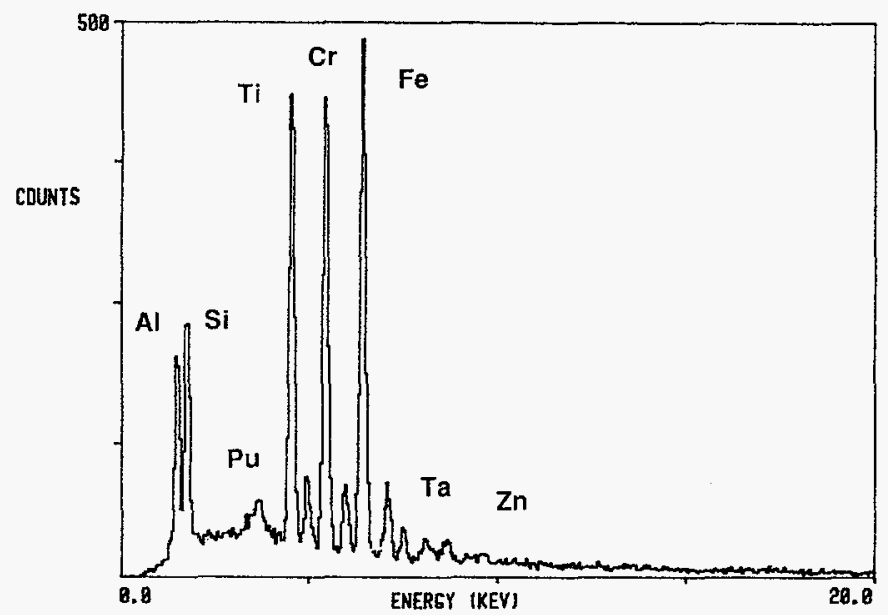

FIGURE 2.3 Energy Dispersive X-ray Spectrum of the Particle Matrix in Fig. 2.2 
particles from this ash heel sample, the matrix was essentially pure aluminum oxide. There were also some particles that were composed entirely of plutonium (see Fig. 2.4) and that were apparently resistant to acid dissolution.

Other examples illustrating the heterogeneous distributions of plutonium within individual particles are shown in Figs. 2.5-2.7. These particles are from a crushed LECO crucible obtained from the Savannah River Site. Their composition is described in Section 2.3.2.

\subsubsection{Survey of Samples}

The microscope samples remained intact during thin sectioning, which preserved the spatial relationships between the phases. Table 2.2 summarizes the results from the AEM study of each of the blocks that were prepared as thin sections. (See Fig. 2.8 for a low-magnification micrograph of a thin section of particles.)

During grinding, the ceramic fraction of the LECO crucible sample was easily reduced in size to -200 mesh $(<74 \mu \mathrm{m})$, while the particles of a metal oxide fraction, composed primarily of copper, remained larger than 200 mesh despite repeated grinding. Figures 2.5 and 2.6 are backscattering electron (BSE) micrographs of particles from the -200 mesh and +200 mesh portions, respectively. In most $(\sim 90 \%)$ of the particles from the -200 mesh fraction, only Si was detected, as evidenced by the EDS image (see Fig. 2.9), while in others, $\mathrm{Al}$ and Si were detected. It is interesting that plutonium was observed only in particles that also contained significant amounts of copper, as shown in Fig. 2.7.

In addition to ash heel and LECO crucibles, other plutonium wastes, such as chloride salts, bomb reduction sand, slag, and other crucibles, have also been shown to be heterogeneous [AVENS], having plutonium distributed as discrete grains within a larger particle matrix.

\subsubsection{Detailed Analysis of Samples}

The following sections describe, in detail, the results of AEM characterization of the materials listed in Table 2.2. The objective of each analysis was to describe both the non-plutoniumbearing particles and the plutonium-bearing particles.

\subsubsection{Los Alamos Ash}

Images of the plutonium particles and ash are shown in Figs. 2.10 and 2.11. We found few differences between the leached and unleached ash samples. There was a suggestion that the mean 


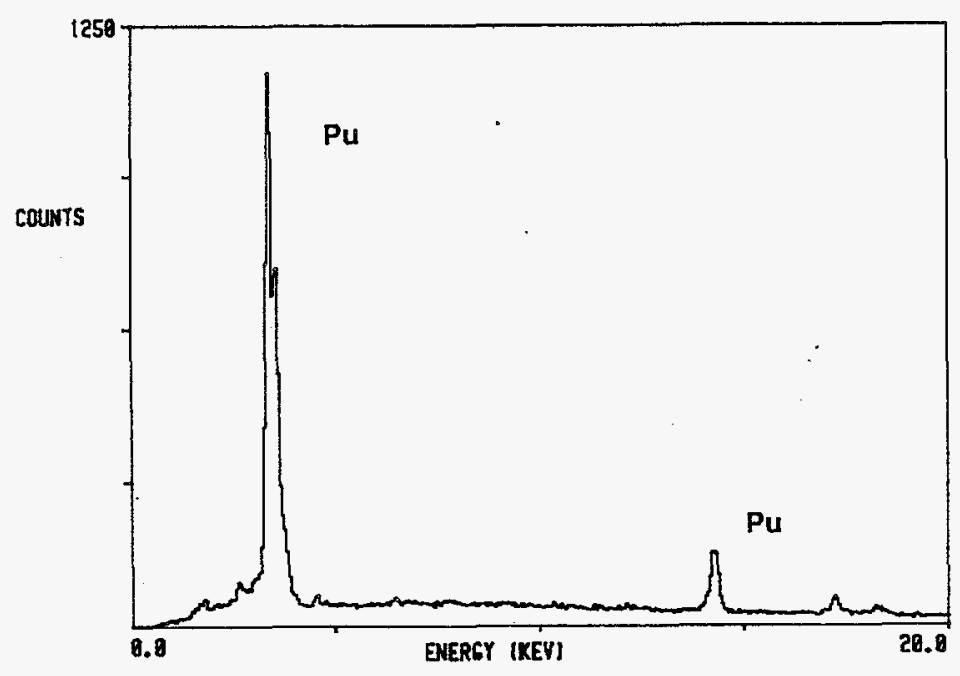

FIGURE 2.4 Energy Dispersive X-ray Spectrum of a

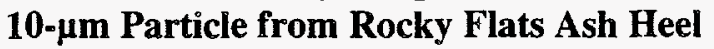

size of the plutonium particles was reduced in the heel sample; however, this is not certain. Furthermore, there were many examples of plutonium particles attached to silicate phases, such as potassium silicate, magnesium silicate, and calcium aluminosilicate phases. Because of the high contrast differences between the plutonium particles and the silicates, we found that photographic film did not always capture all the particles, so we used the CCD camera, which has much better linearity of detection.

Diffraction analysis and EDS analysis were performed on the plutonium oxide phases to establish the type of oxide (see Table 2.3 and Fig. 2.11). The results obtained were compared to literature values from three cubic-plutonium oxides: $\mathrm{Pu}_{4} \mathrm{O}_{7}$, nonstoichiometric $\mathrm{PuO}_{2-\mathrm{x}}$, and $\mathrm{PuO}_{2}$. Some of the matches with $\mathrm{Pu}_{4} \mathrm{O}_{7}$ could be explained by the fact that there are many more reflections from this phase than from the other plutonium oxides; therefore, there is a greater chance that any $\mathrm{d}$-spacing obtained will match with a d-spacing from this phase. Moreover, the presence of some of the larger d-spacings (i.e., $3.89 \AA$ and $3.12 \AA$ ) indicates that the phase is not $\mathrm{PuO}_{2}$ but is a nonstoichiometric plutonium oxide.

\subsubsection{Rocky Flats Ash}

In Figs. 2.12 and 2.13, TEM images, electron diffraction patterns, and EDS spectra from Rocky Flats ash are shown. The particles were typically between 10 and $70 \mathrm{~nm}$ in diameter.

A partial Kikuchi map produced by tilting around one of the small plutonium oxide particles indicated that this phase is cubic, which is in agreement with the other diffraction data (see Fig. 2.14a). In addition, EELS was performed on the plutonium phase, as shown in Fig. 2.14b. The 

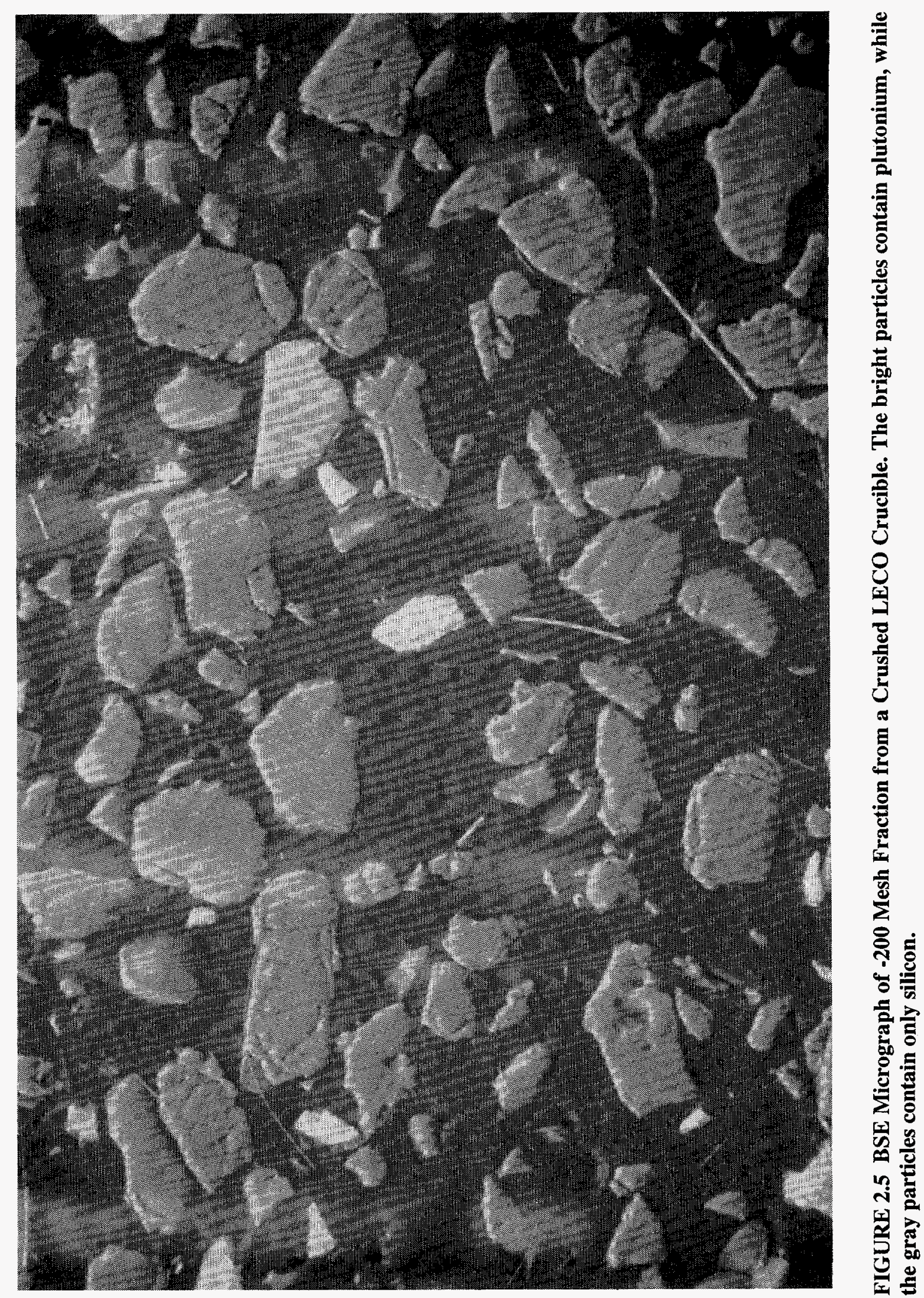


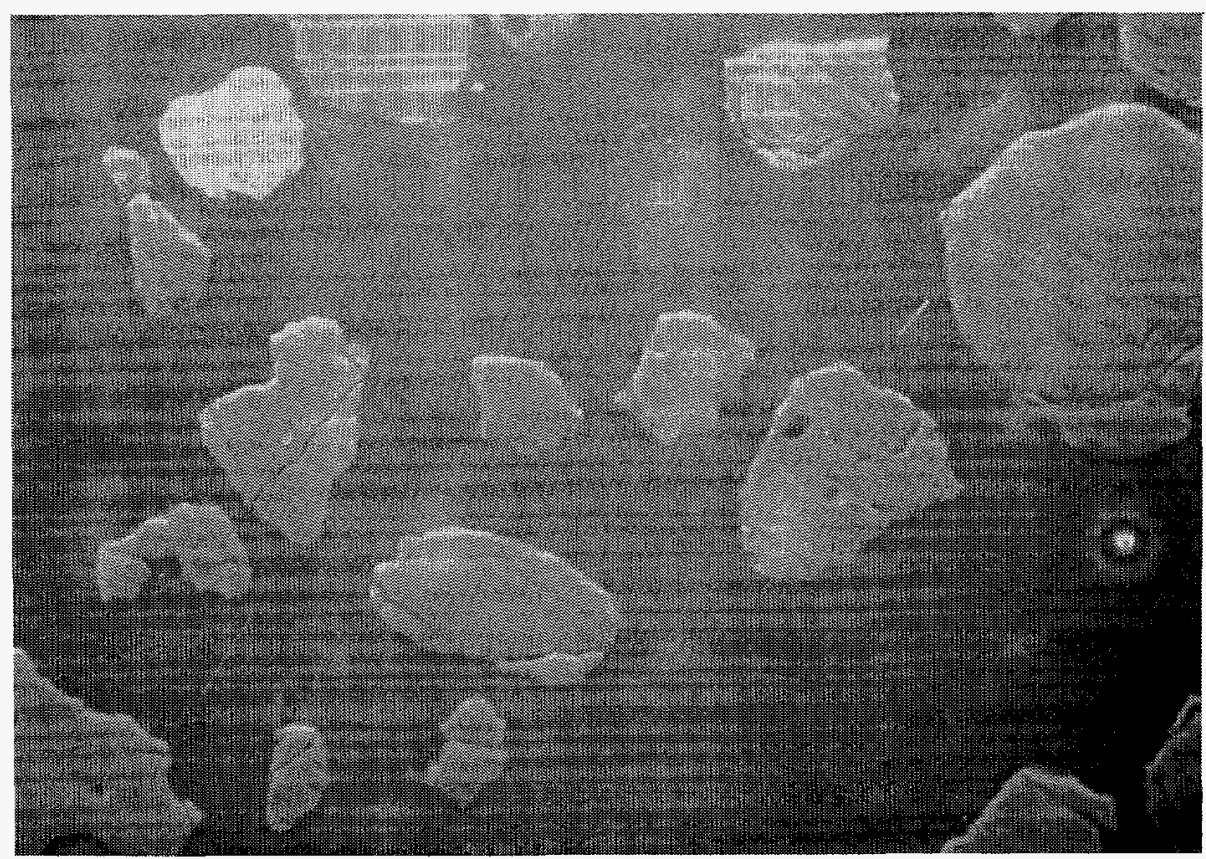

FIGURE 2.6 BSE Micrograph of +200 Mesh Fraction from a Crushed LECO Crucible

EDS analyses of a number of the particles suggested that phases other than plutonium oxide were present; however, this was not certain. In Fig. 2.13, the EDS analysis of an aluminosilicate is shown; in this case also it was not clear whether this phase was incorporated into the silicate, or whether the $\mathrm{X}$-ray signal was due to two phases. However, analysis of Rocky Flats ash heel samples revealed a similar composition in a glassy phase, which was determined to be a plutonium-bearing glass (see Section 2.3.3.3). Note that titanium was found in the Rocky Flats ash samples (Fig. 2.13) but not in the Los Alamos samples.

A number of other phases were found in the ash samples from Rocky Flats, including silicates $\left(\mathrm{CaAlSiO}\right.$. [possibly anorthite], $\mathrm{SiO}_{2}$ [quartz], and $\mathrm{CaMgSiO}_{\mathrm{x}}$ ) and zirconia (an artifact from the grinding process). Figure 2.13 shows the EDS of an aluminosilicate found in the Rocky Flats ash samples. These phases were generally larger than the plutonium particles. Plutonium was found attached to these silicate particles. Many of the various aluminosilicate phases were amorphous.

\subsubsection{Rocky Flats Ash Heel}

We received incinerator ash and ash heel samples from Rocky Flats Plant and an incinerator ash sample from Los Alamos. Such wastes constitute the major part of the plutonium-bearing wastes in storage. Characterization of these wastes is extremely important in the effort to develop methods 


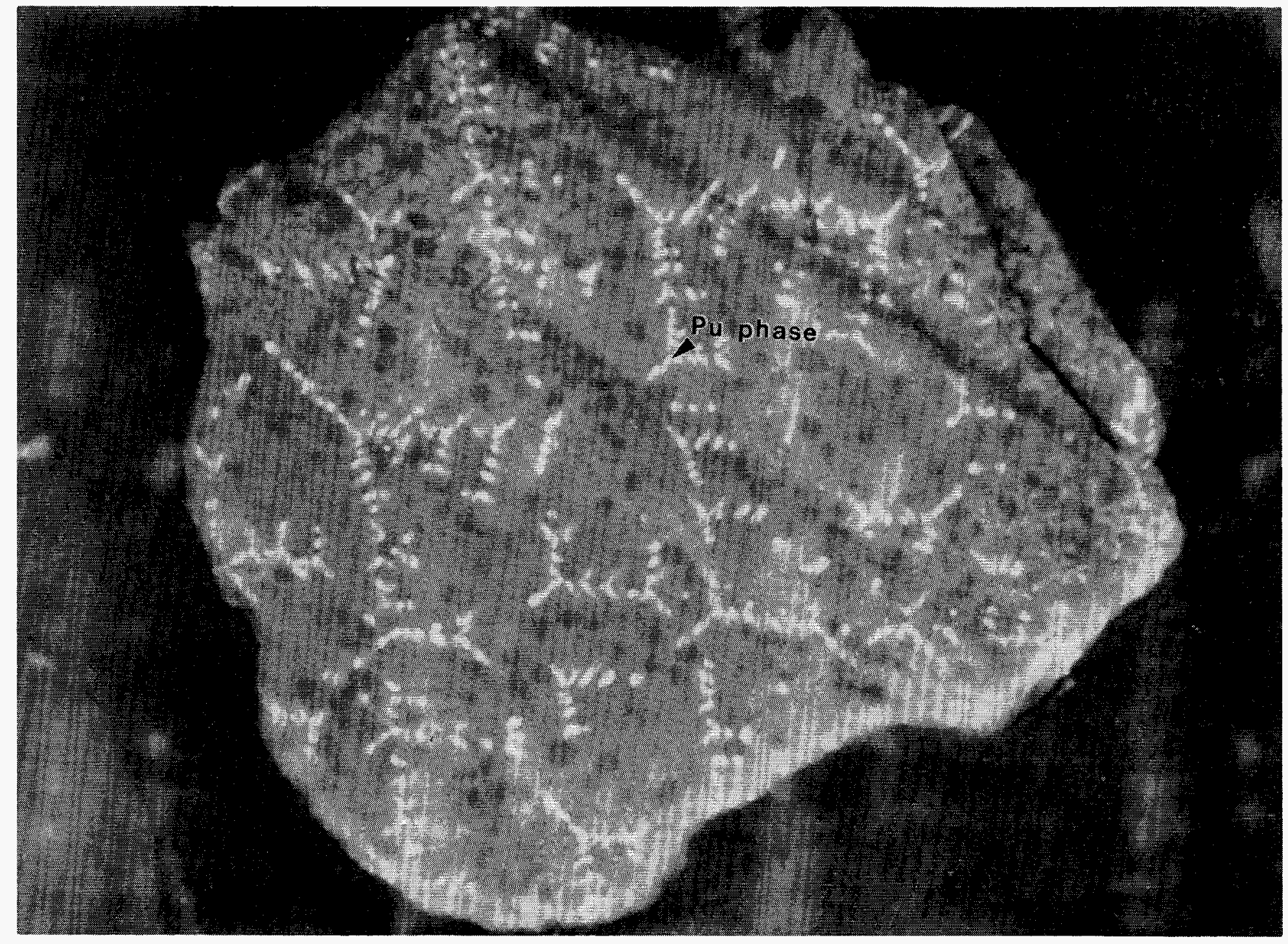

FIGURE 2.7 BSE Image of Plutonium-Bearing Particles from a Crushed LECO Crucible. The brightest spots contain $\mathrm{Pu}$, the grey matrix contains $\mathrm{Cu}$, and the darkest regions contain $\mathrm{Al}$. Note how the plutonium particles are arranged along the grain boundaries. Note also the presence of either a eutectic or a peritectic near the edge of the particle. 


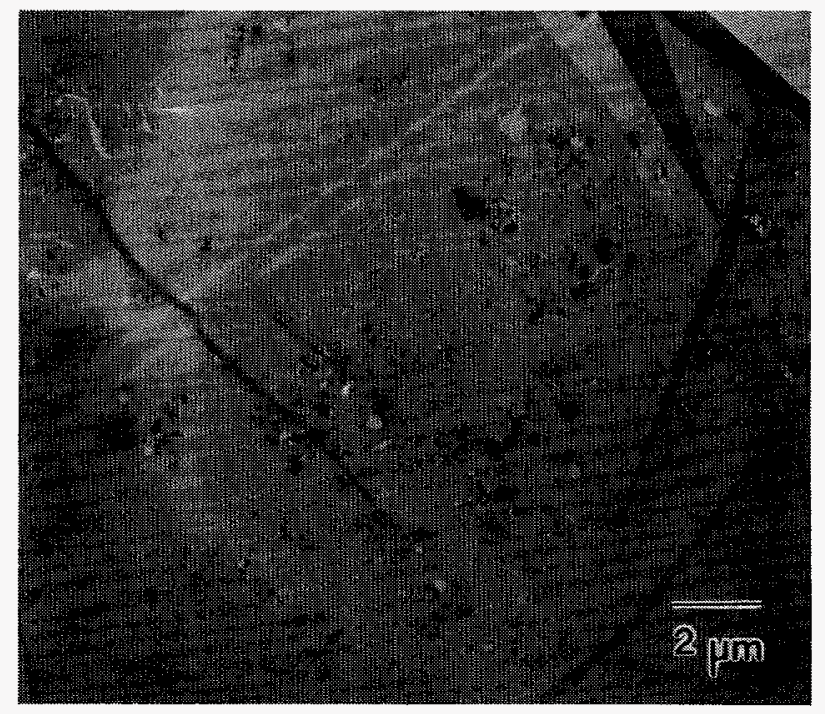

FIGURE 2.8 Low Magnification Micrograph of Thin Section of Plutonium and Other Particles.

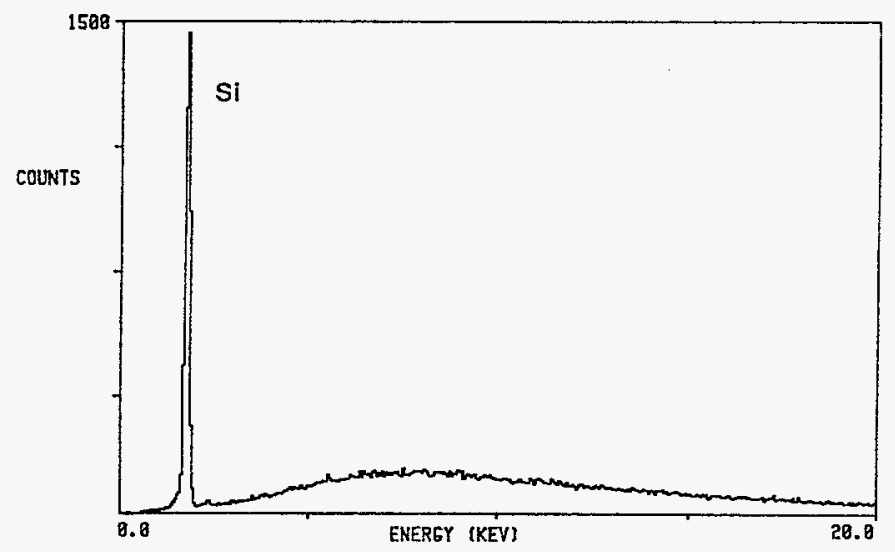

FIGURE 2.9 Typical Energy Dispersive X-ray Spectrum of a -200 Mesh Particle from a Crushed LECO Crucible 

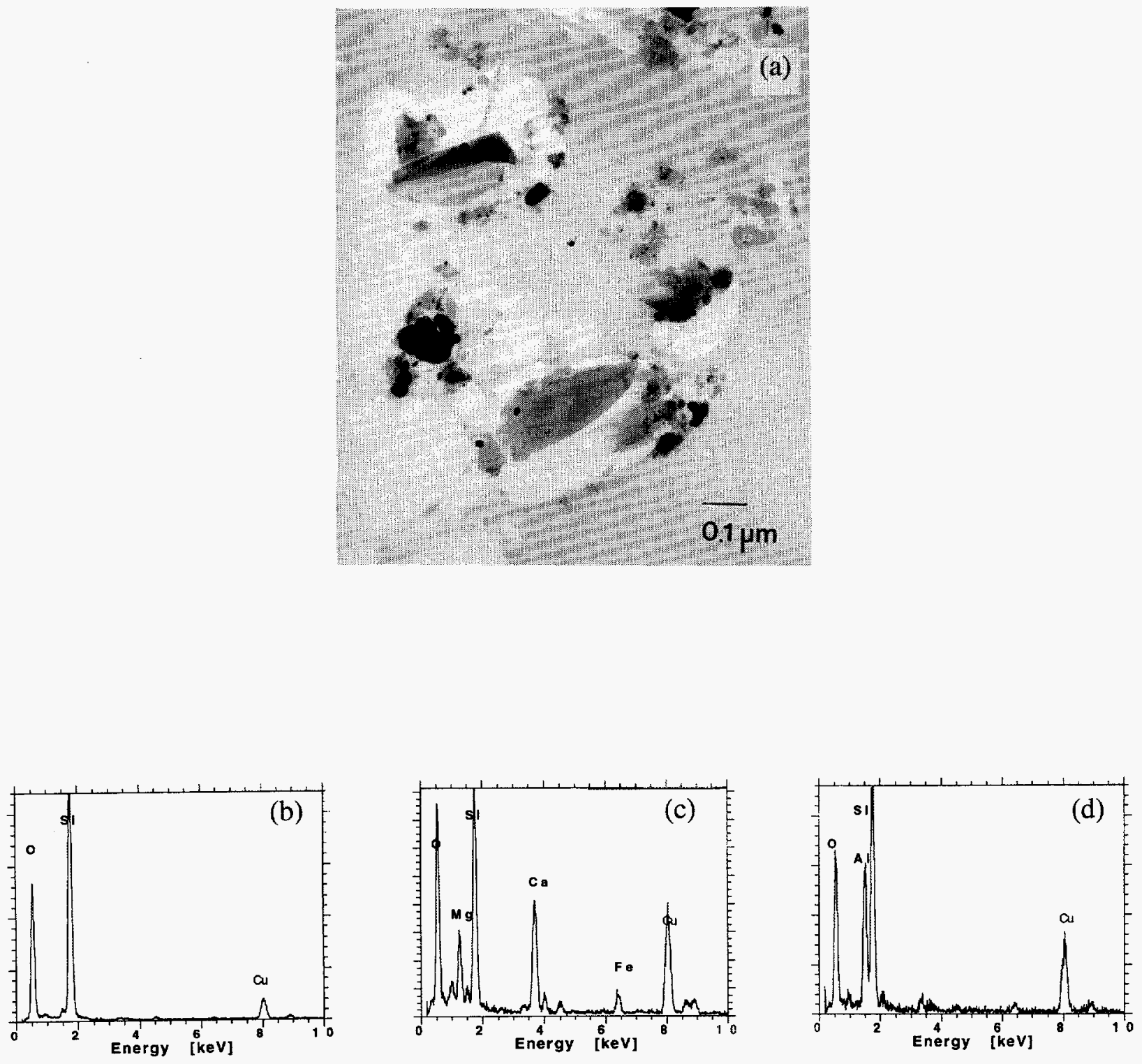

FIGURE 2.10 General Analysis of Los Alamos Ash. (a) Low-magnification image of particles. (b-d) EDS analysis of non-plutonium-bearing particles: (b) quartz; (c) a calcium magnesium silicate (possibly diopside); (d) an unidentified aluminosilicate. 


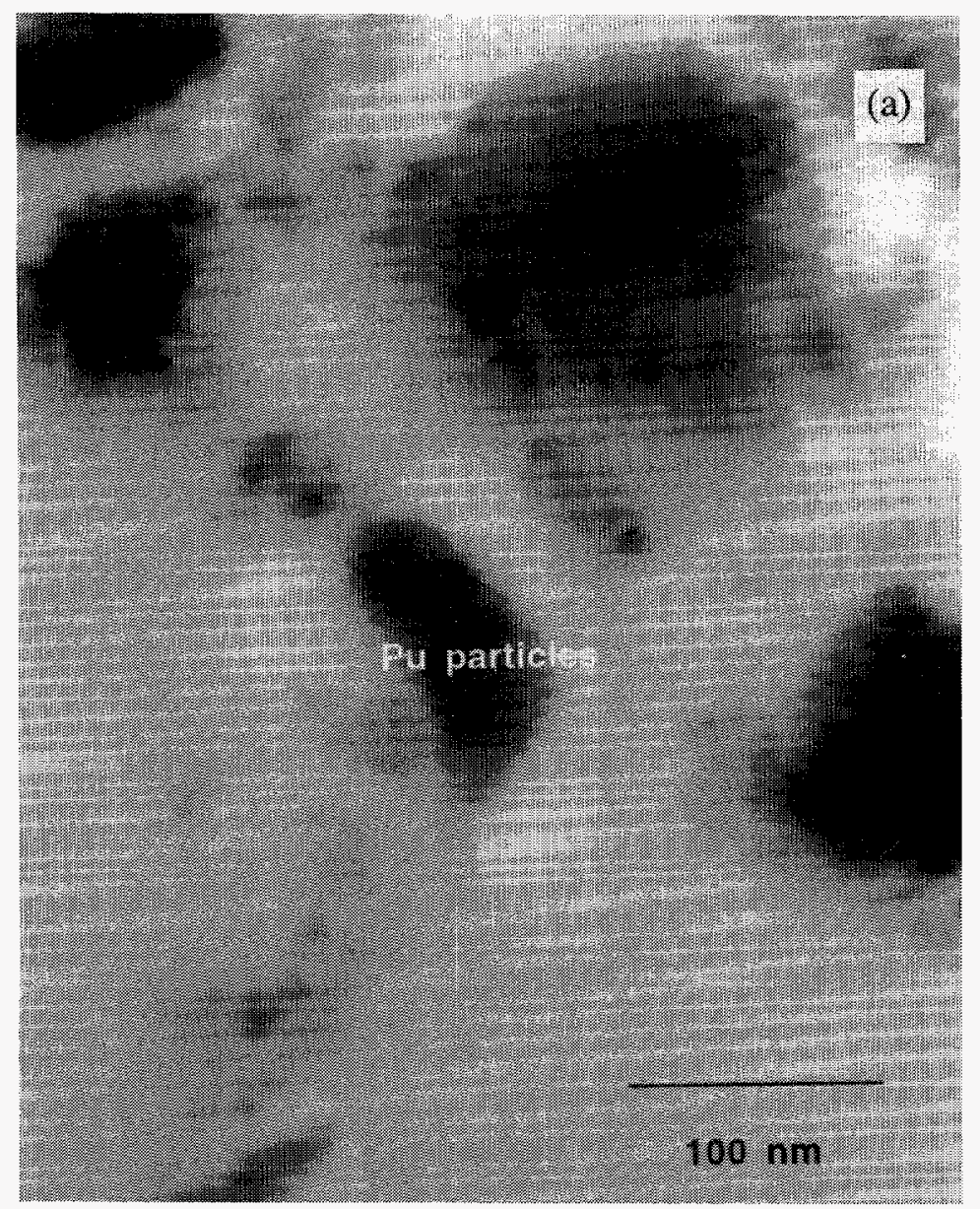

FIGURE 2.11 Analysis of Plutonium Oxide Particles in Los Alamos Ash. (a-b) TEM images of plutonium oxide and other particles. The plutonium grains were around $10-20 \mathrm{~nm}$ in diameter and were usually attached to larger silicate particles. (Figure and caption continued.) 

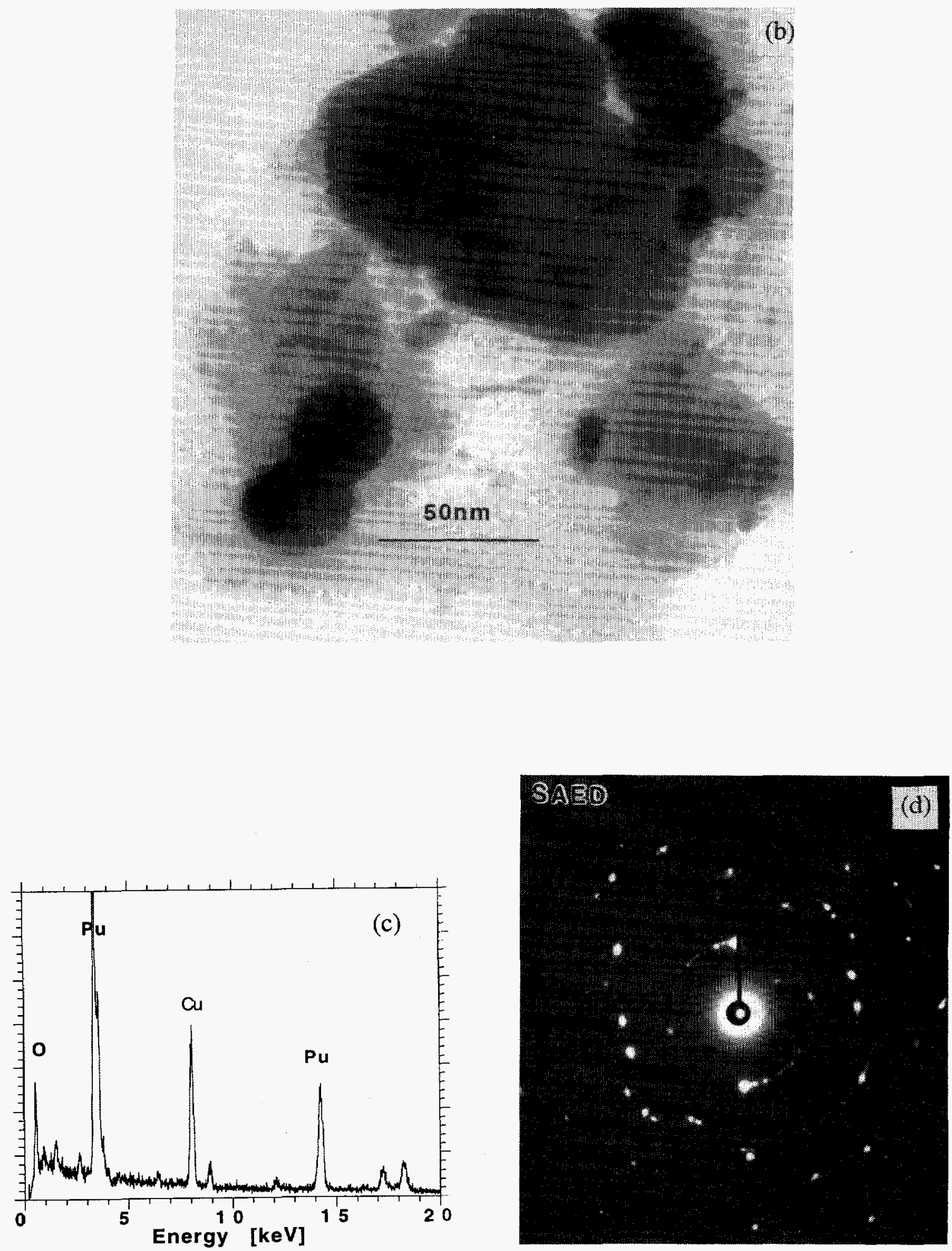

FIGURE 2.11 (Cont.) (c) EDS analysis of plutonium oxide phase. The $\mathrm{Cu}-\mathrm{K}_{\alpha, \beta}$ peak is due to fluorescence from the copper grid. The low oxygen signal is due to the severe adsorption of the low energy $\mathrm{O}-\mathrm{K} \mathrm{X}$-ray line. (d) Electron diffraction pattern from plutonium oxide particle. 
TABLE 2.3 Electron Diffraction Analysis from

Plutonium Oxide Particles in Los Alamos Ash ${ }^{\mathrm{a}}$

\begin{tabular}{|c|c|c|c|}
\hline \multirow[b]{2}{*}{$\begin{array}{l}\text { Experimental } \\
\text { d-Spacings, } \AA\end{array}$} & \multicolumn{3}{|c|}{ Literature d-Spacings, $\AA$} \\
\hline & $\mathrm{Pu}_{4} \mathrm{O}_{7}$ & $\mathrm{PuO}_{2-\mathrm{x}}$ & $\mathrm{PuO}_{2}$ \\
\hline 4.89 & & & \\
\hline 3.87 & 3.89 & & \\
\hline 3.12 & 3.18 & 3.14 & 3.11 \\
\hline $2.97,2.86$ & 2.94 & & \\
\hline 2.77 & 2.755 & 2.72 & \\
\hline 2.62 & 2.60 & & 2.69 \\
\hline 2.37 & 2.35 & & \\
\hline 2.27 & 2.249 & & \\
\hline $1.95,1.91$ & 1.95 & 1.92 & 1.9 \\
\hline 1.84 & 1.84 & & \\
\hline $1.71,1.71$ & 1.7004 & & \\
\hline 1.639 & 1.6248 & 1.64 & 1.627 \\
\hline 1.60 & 1.59 & 1.57 & 1.62 \\
\hline 1.298 & 1.298 & & \\
\hline 1.136 & 1.1366 & & \\
\hline 1.09 & 1.0911 & 1.11 & 1.03 \\
\hline 0.94 & 0.945 & 0.96 & 0.9539 \\
\hline 0.76 & & 0.78 & 0.7788 \\
\hline
\end{tabular}

a Self-irradiation of the plutonium oxide will result in expansion of the lattice $\left({ }^{239} \mathrm{PuO}_{2}\right.$ expands at around $0.0073 \AA$ A/yr) [CLEVELAND-1979].

to remove and immobilize the plutonium. The particles from the Rocky Flats ash heels proved to be the most varied of all the samples examined. The BSE image of the particles shows that the plutonium (bright regions in Fig. 2.15), although mainly concentrated, is also spread diffusely throughout the particle matrix, and not along specific grain boundaries, as was shown in Fig. 2.7 for a crushed LECO crucible sample.

As in the ash samples, the major plutonium-bearing phase was a reduced oxide (see Table 2.4 and Fig. 2.15); however, other types of plutonium were also found. Table 2.5 and Fig. 2.16 show a plutonium titanium silicate phase. Because the database on plutonium-bearing phases is extremely limited, techniques such as AEM, which is the only method for identifying unique unknowns, must be used to expand the knowledge base. The EDS data in Table 2.5 show that the 


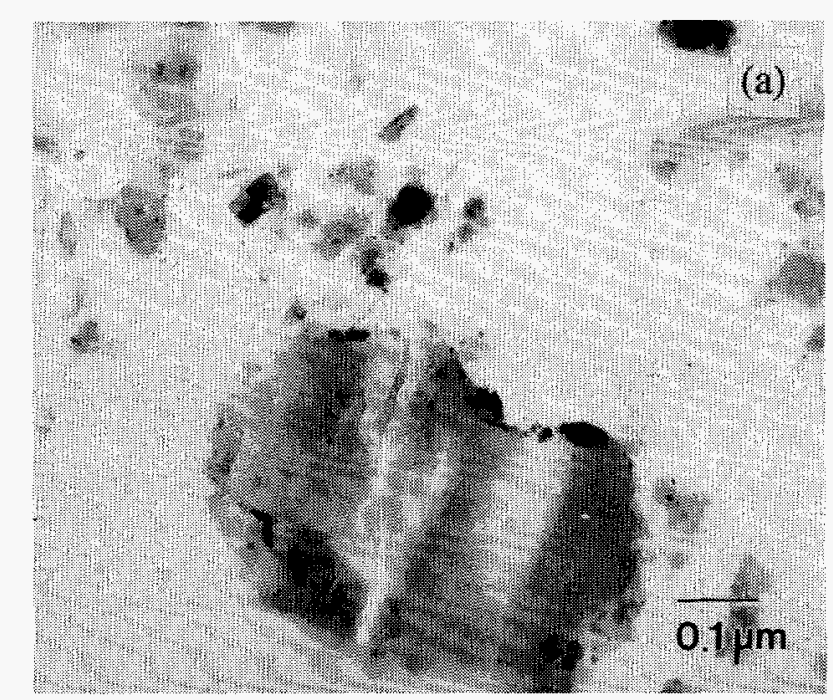

\section{FIGURE 2.12 Analysis of Plutonium Oxide Particles in Rocky Flats Ash. (a-d) TEM images of plutonium oxide and other particles. Plutonium particles have collected along the edges of the larger silicate particles. (Figure and caption continued.)}

match with the actinide orthosilicate, plutonium silicate $\left(\mathrm{PuSiO}_{4}\right)$, is not particularly good. The match with the smaller d-spacings may be due to similar Si-O and $\mathrm{Pu}-\mathrm{O}$ bond lengths. Thoriumbearing (thorite) and uranium-bearing (huttonite) actinide orthosilicates are widespread in nature in igneous and metamorphic rocks.

There were a number of non-plutonium-bearing crystalline phases such as aluminum oxides (corundum) and an iron-chromium oxide phase (cubic chromite, a spinel-group mineral phase) that did not contain a measurable amount of plutonium. The electron diffraction data from these phases are listed in Table 2.6.

The most favored option for dealing with disposal of waste weapons-grade plutonium, at sites such as Rocky Flats, is vitrification to form a glass [GRAY-1995]. Interestingly, a plutoniumbearing glassy silicate phase was identified in ash heel samples in this study (see Fig. 2.17). These types of silicate particles were commonly observed in the Rocky Flats ash heel samples. They were completely amorphous, with plutonium spread throughout the particle, but enriched to around $75 \mathrm{wt} \%$ in some regions. The darker area in Fig. 2.17 corresponds to the plutonium-enriched region. The rounded shape of the particle suggests that this is a glassy phase and not the result of metamictization of a crystalline one. The EDS analysis of the phase, detailed in Table 2.7, indicates that the silicate phase contained a significant amount of plutonium, close to $25 \mathrm{wt} \%$ of the EDS-detectable elements (boron and lithium may also be present). Incineration may have caused plutonium and silica to react to form the glass. A similar phase has been observed during incineration 


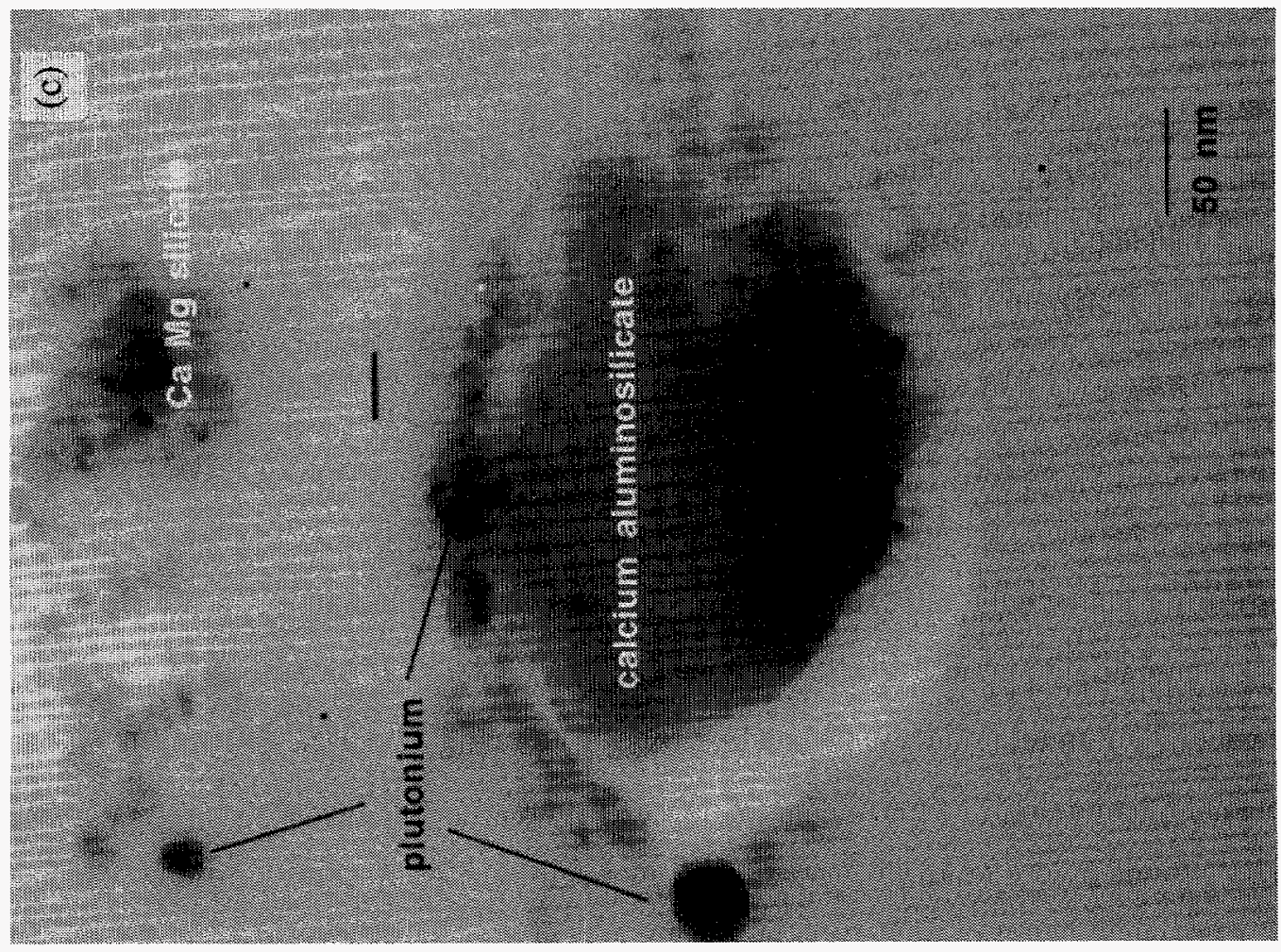

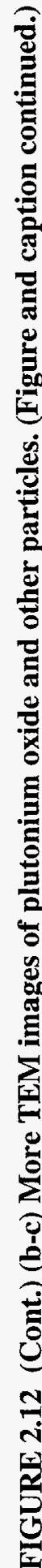

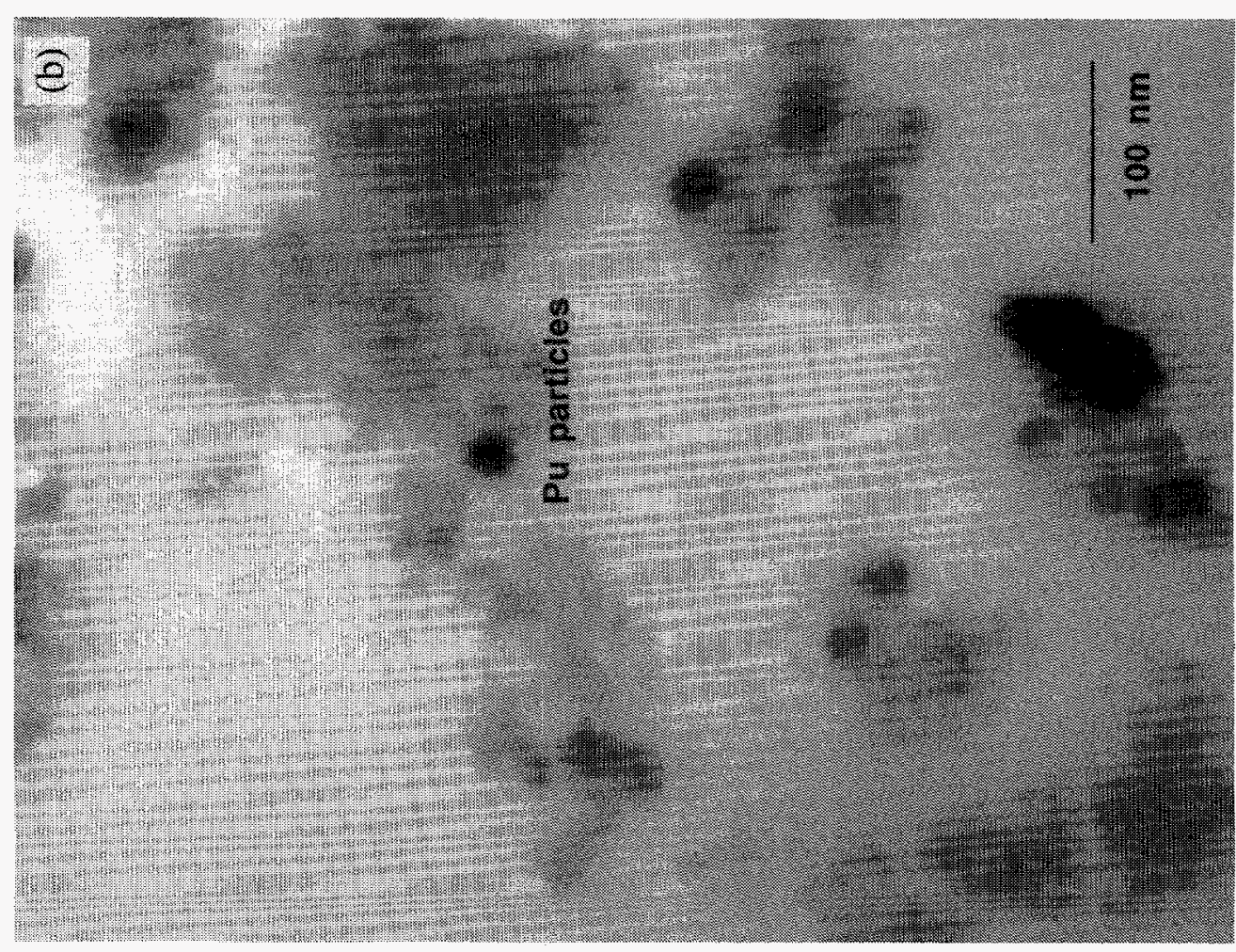



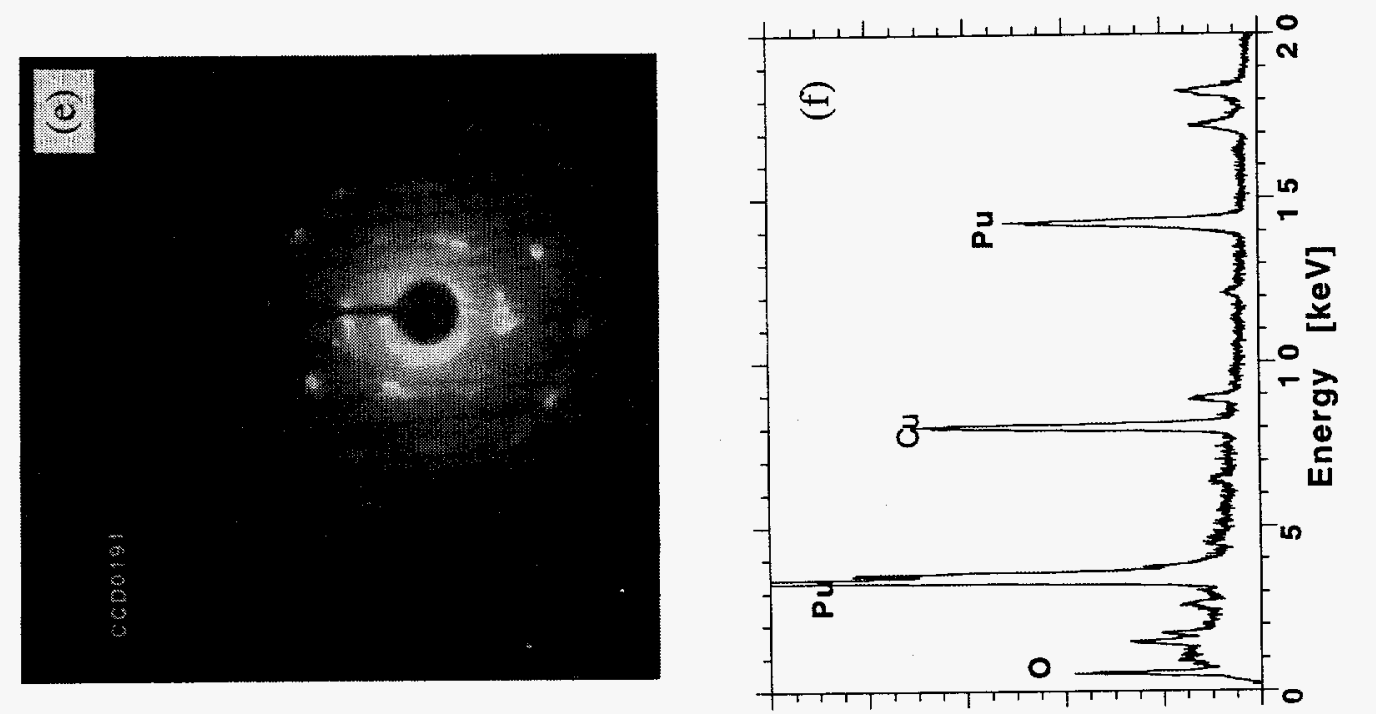

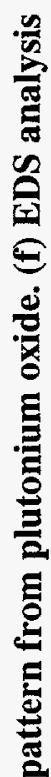

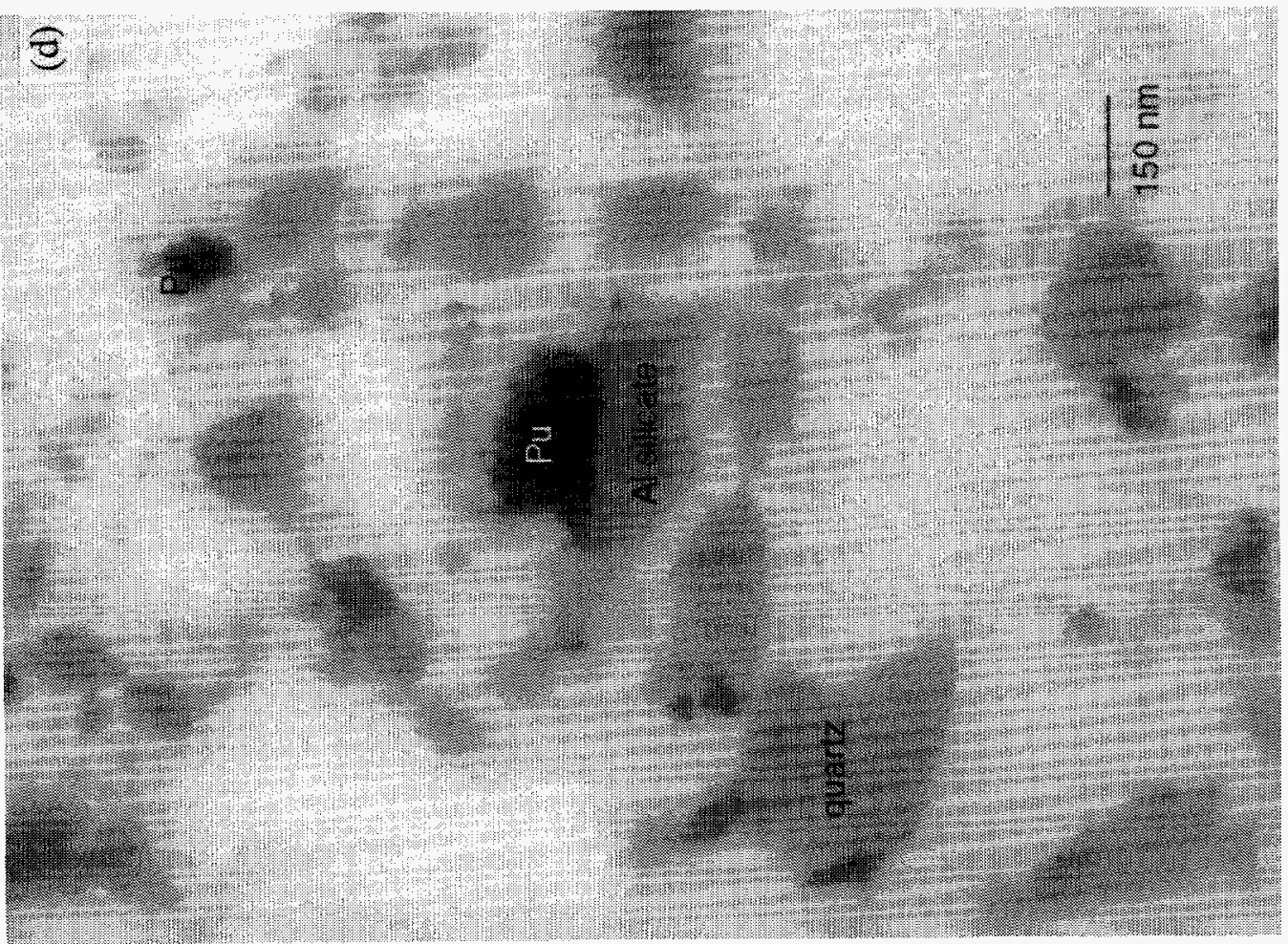

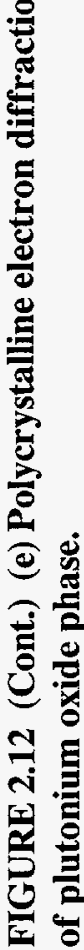



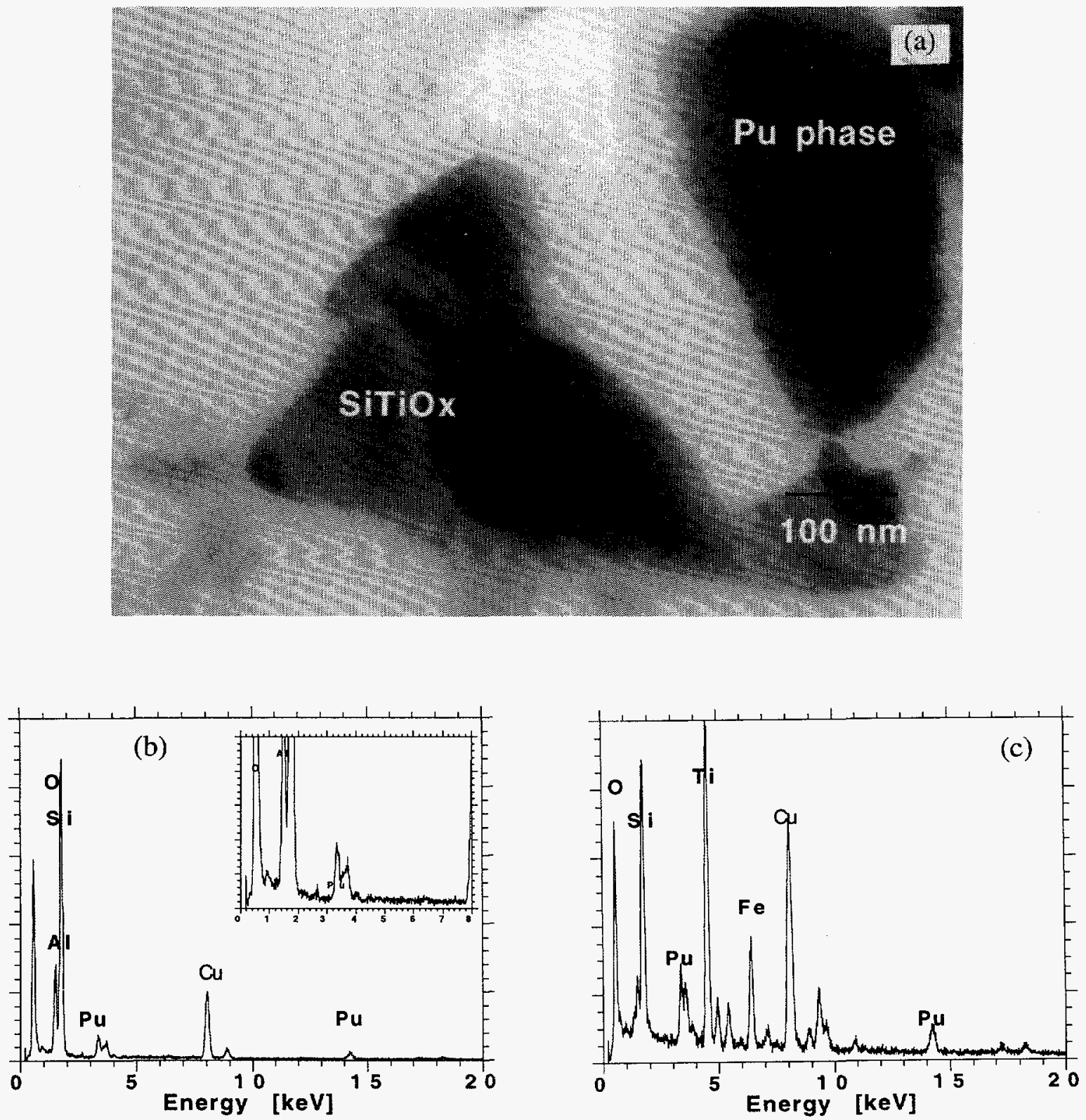

FIGURE 2.13 Further Analysis of Particles in Rocky Flats Ash. (a) TEM image of plutonium phase and silicotitanate particle. (b-d) EDS analyses of plutonium-bearing particles: (b) plutonium glass; (c-d) possible plutonium silicotitanate phase. (Figure and caption continued.) 

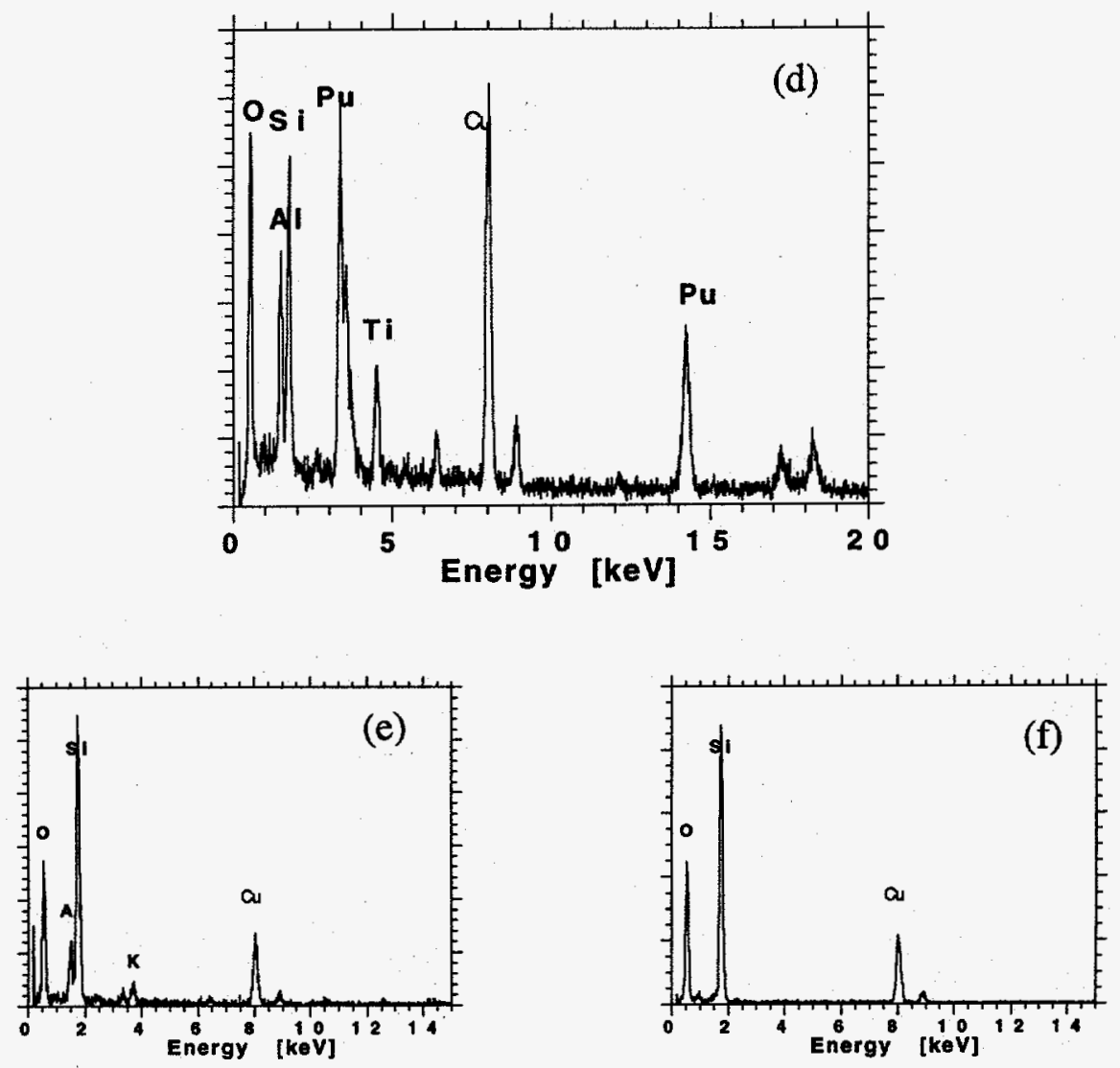

FIGURE 2.13 (Cont.) (e-f) EDS analyses of non-plutonium-bearing particles: (e) potassium aluminosilicate; (f) quartz. 


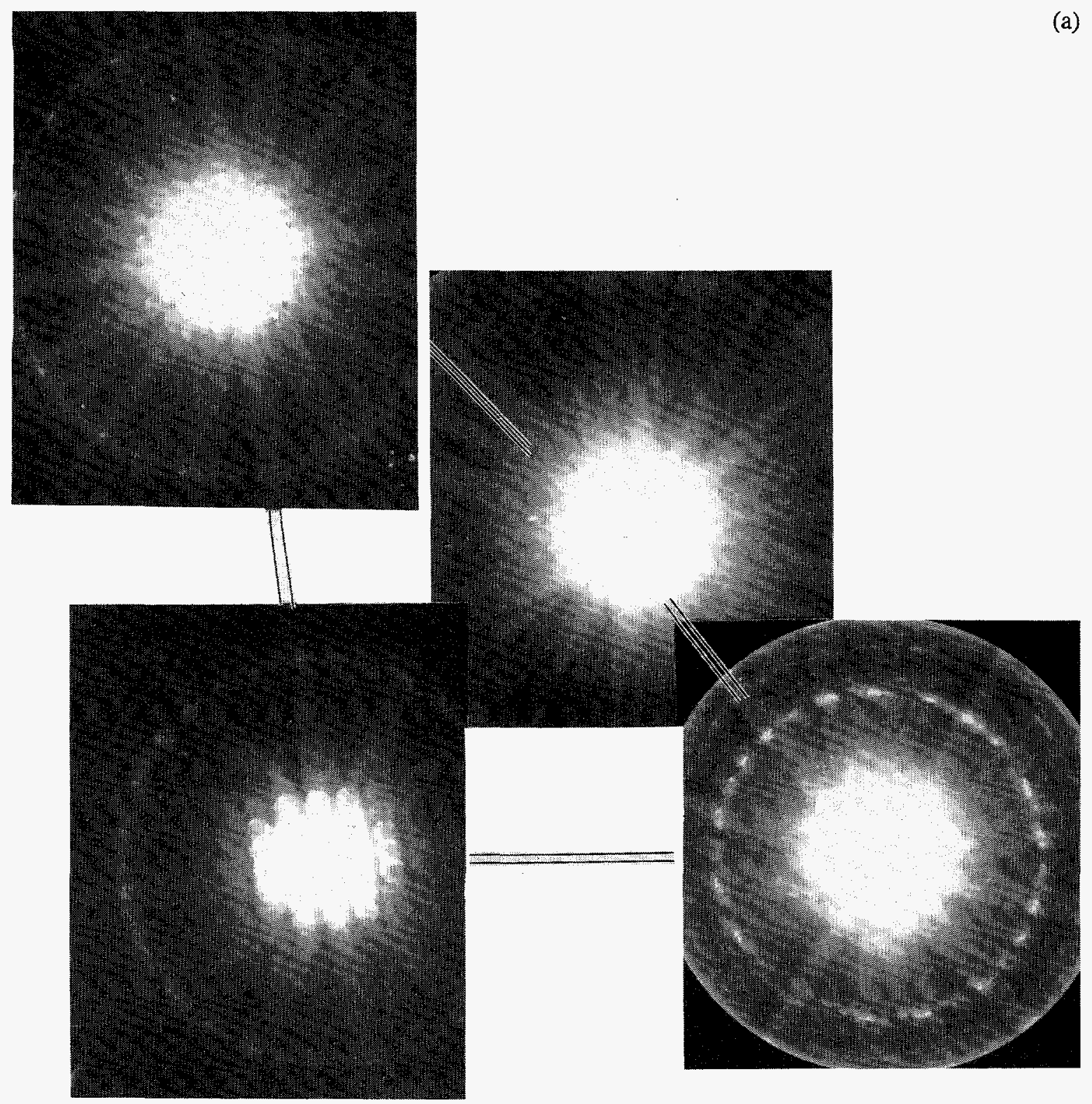

FIGURE 2.14 (a) Partial Kikuchi Map of Plutonium Oxide in Rocky Flats Ash (shows the phase is cubic) and (b) EELS Analysis of the Pu-O $\mathrm{O}_{4,5}$ Edges 


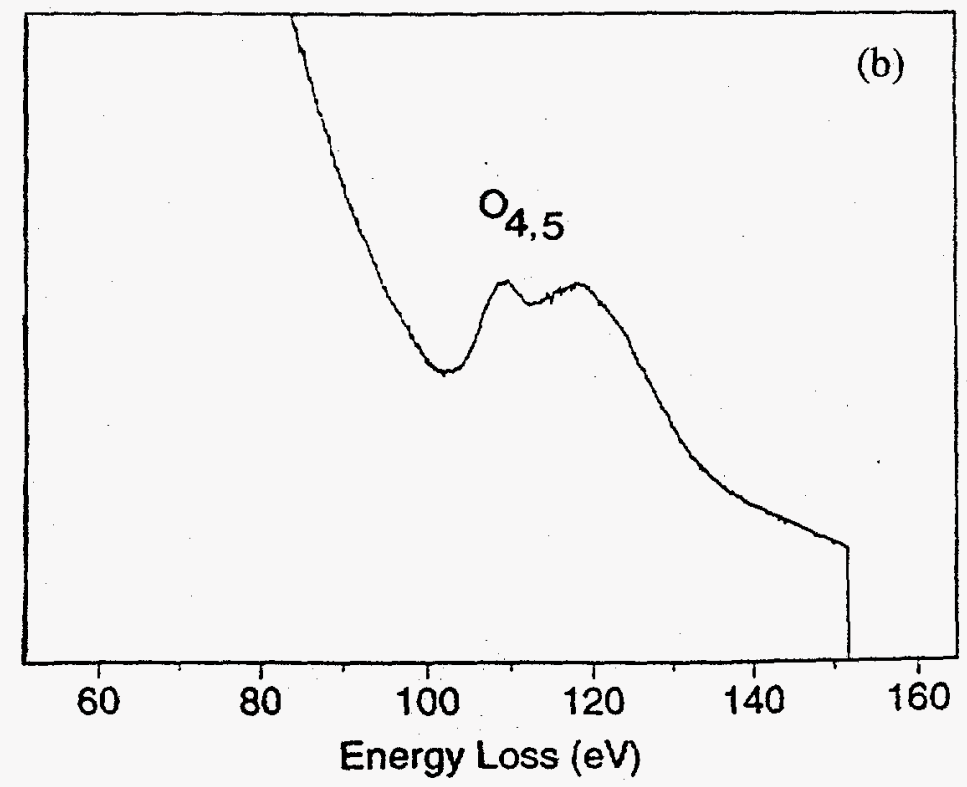

FIGURE 2.14 (Cont.) 


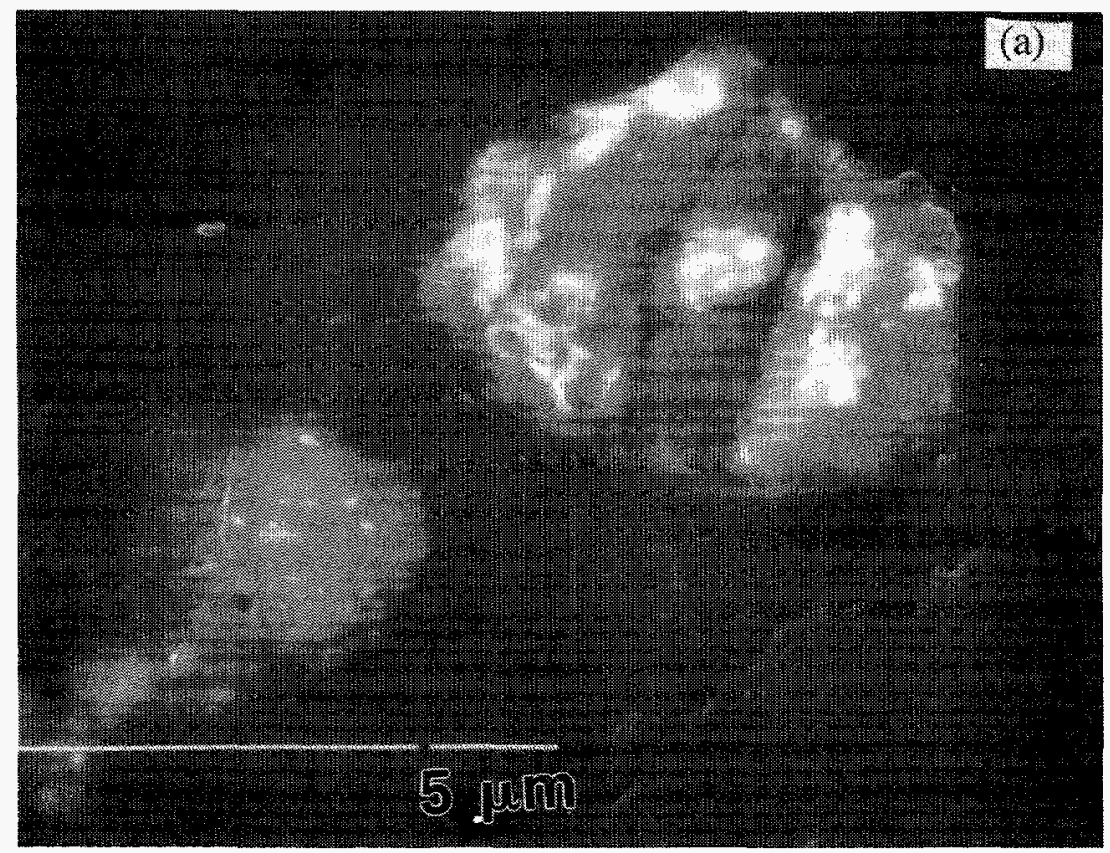

FIGURE 2.15 Analyses of Plutonium-Bearing and Non-PlutoniumBearing Particles from Rocky Flats Ash Heels. (a) BSE image of uncrushed plutonium. (Figure and caption continue.) 


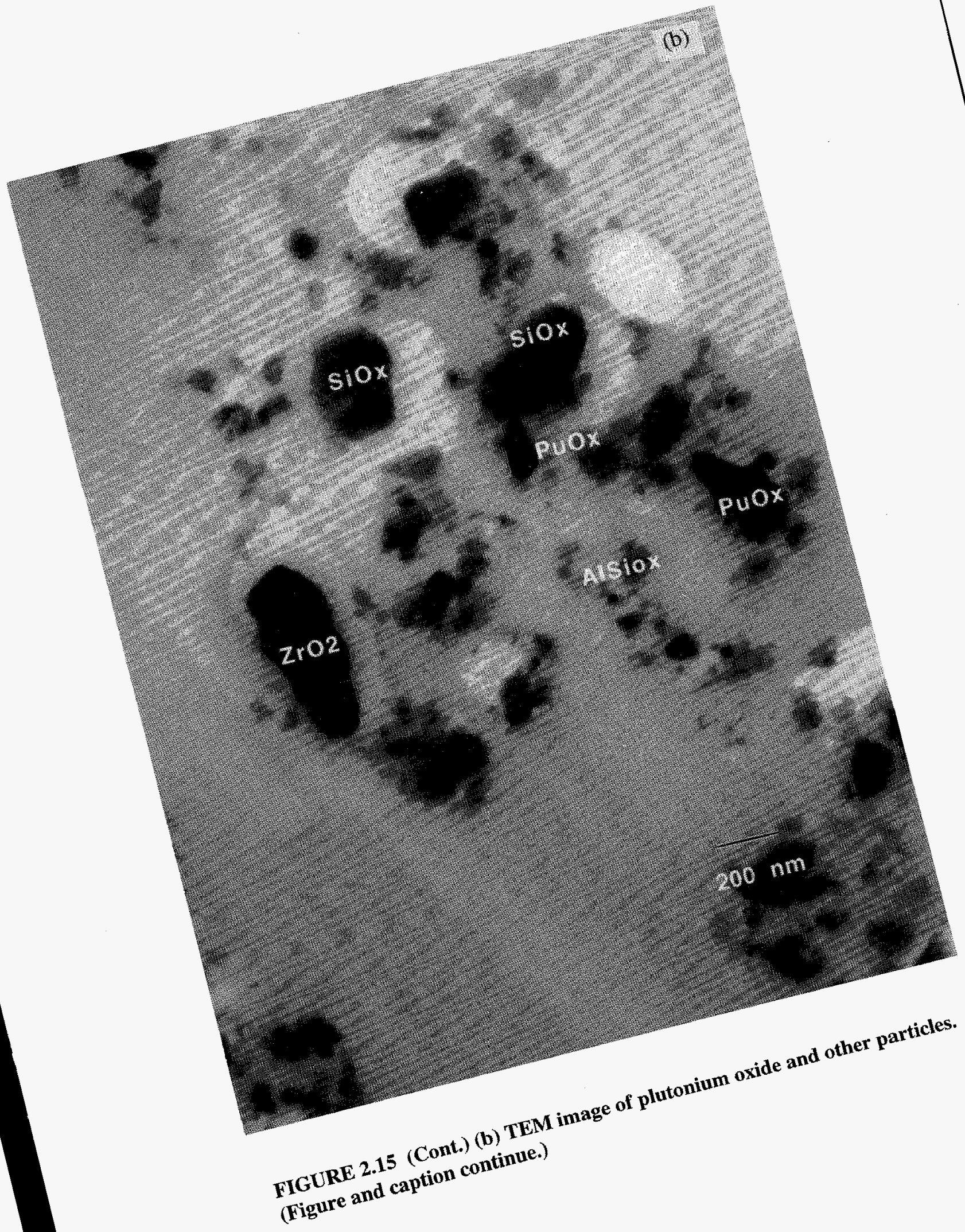



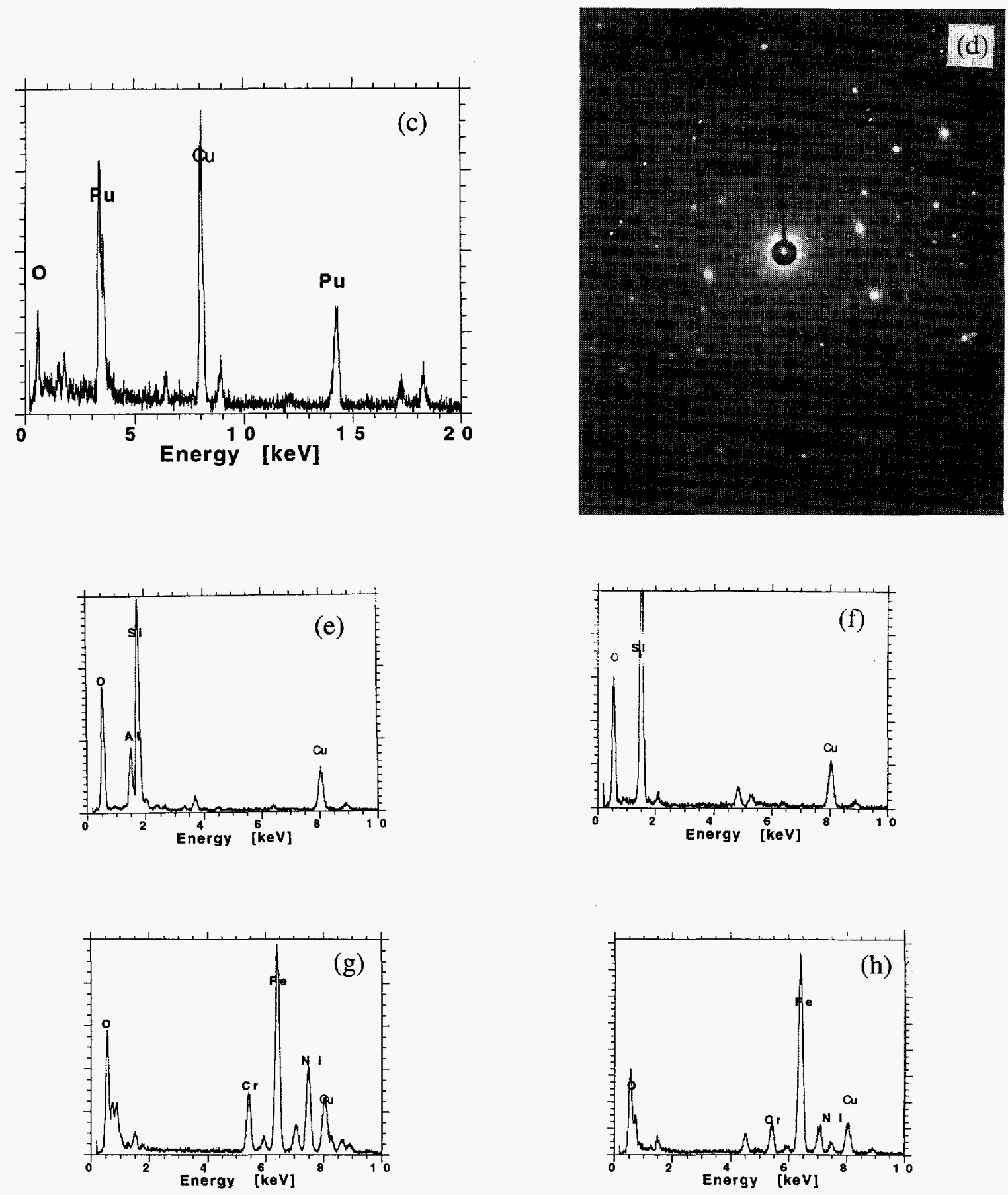

FIGURE 2.15 (Cont.) (c) EDS analysis of the plutonium oxide phase. (d) A polycrystalline electron diffraction pattern. (e-h) EDS analysis of non-plutonium-bearing particles: (e) an aluminosilicate phase; (f) quartz; (g) chromite; (h) iron oxide. 
TABLE 2.4 Electron Diffraction from Plutonium Oxide Particles in Rocky Flats Ash Heel

\begin{tabular}{cccc}
\hline & \multicolumn{3}{c}{ Literature d-Spacings, $\AA^{\mathrm{a}}$} \\
\cline { 2 - 4 } $\begin{array}{c}\text { Experimental } \\
\text { d-Spacings, } \AA\end{array}$ & $\mathrm{Pu}_{4} \mathrm{O}_{7}$ & $\mathrm{PuO}_{2-\mathrm{x}}$ & $\mathrm{PuO}_{2}$ \\
\hline & & & \\
3.18 & 3.18 & 3.14 & 3.11 \\
2.00 & 1.95 & & \\
1.76 & 1.79 & & \\
1.58 & 1.59 & 1.57 & 1.62 \\
\hline
\end{tabular}

a From [ROOF].

TABLE 2.5 Electron Diffraction from Crystalline Plutonium Phases in Rocky Flats Ash Heel

\begin{tabular}{|c|c|c|}
\hline \multicolumn{2}{|c|}{$\begin{array}{l}\text { Experimental } \\
\text { d-Spacings, } \AA\end{array}$} & \multirow{2}{*}{$\begin{array}{c}\text { Literature } \\
\text { d-Spacings, } \AA \\
\text { PuSiO }_{4} \\
\text { (JCPDS 43-1095) }\end{array}$} \\
\hline Pu-Si-Ti phase & Al-Pu phase & \\
\hline \multicolumn{3}{|l|}{$6.10,6.10$} \\
\hline \multicolumn{3}{|l|}{$5.50,5.32$} \\
\hline 4.83 & & 4.62 \\
\hline 3.72 & & 3.453 \\
\hline \multicolumn{3}{|l|}{$3.17,3.17$} \\
\hline $2.51,3.04$ & 2.70 & $2.63,2.767$ \\
\hline $2.03,2.30,2.40,2.42$ & & $2.441,1.986$ \\
\hline \multirow[t]{3}{*}{$1.85,1.83$} & & 1.83 \\
\hline & 1.76 & 1.787 \\
\hline & & 1.722 \\
\hline $1.59,1.52$ & & 1.55 \\
\hline
\end{tabular}



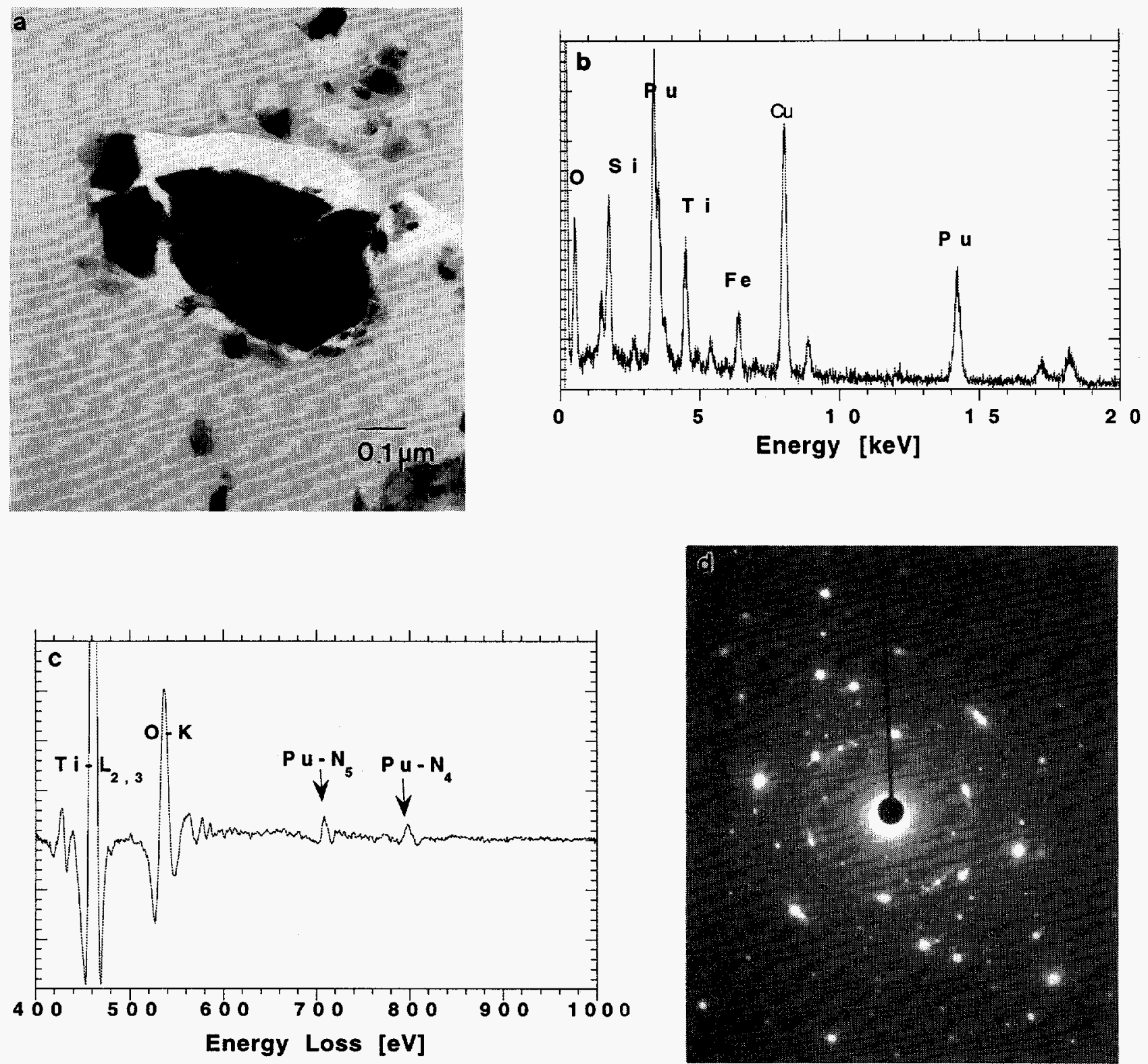

FIGURE 2.16 Analyses of Plutonium Titanium Silicate Phase from Rocky Flats Ash Heel. (a) TEM image. (b) EDS analysis. (c) EELS showing the $\mathrm{Pu}-\mathrm{N}_{4,5}$ edges, as well as $\mathrm{Ti}-\mathrm{L}_{2,3}$ edges and the $\mathrm{O}-\mathrm{K}$ edge. (d) Electron diffraction pattern (d-spacings listed in Table 2.5). 
TABLE 2.6 Electron Diffraction from Non-Plutonium-Bearing Phases in Rocky Flats Ash Heel

\begin{tabular}{ccccc}
\hline $\begin{array}{c}\text { Experimental } \\
\text { d-Spacings, } \AA\end{array}$ & $\begin{array}{c}\text { Literature } \\
\text { Values for } \\
\text { Aluminum Oxide }\end{array}$ & $\begin{array}{c}\text { Experimental } \\
\text { d-Spacings, } \AA\end{array}$ & $\begin{array}{c}\text { Experimental } \\
\text { d-Spacings, } \\
\text { Iron Chromium } \\
\text { Oxide Particle }\end{array}$ & $\begin{array}{c}\text { Literature } \\
\text { Values } \\
\text { for Chromite } \\
\text { (JCPDS 3-873) }\end{array}$ \\
\hline 3.21 & & 3.04 & 4.90 & 4.82 \\
2.81 & & & 2.98 & 2.95 \\
2.60 & 2.55 & 1.85 & 2.51 & 2.52 \\
2.14 & 2.165 & & 2.44 & 2.40 \\
1.69 & 1.74 & & 1.72 & 1.69 \\
1.43 & 1.40 & & 1.63 & 1.60 \\
\hline
\end{tabular}



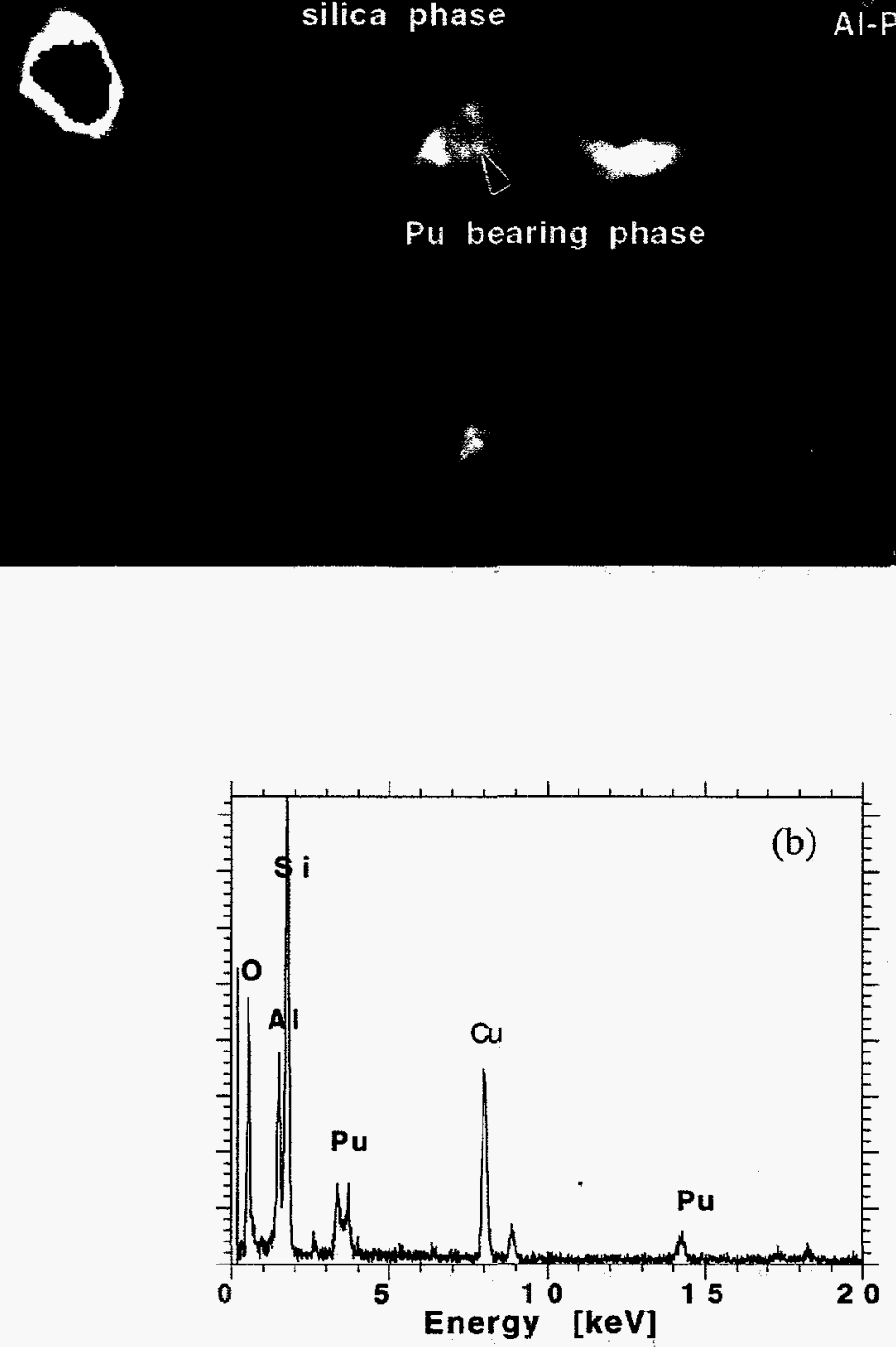

FIGURE 2.17 (a) TEM Image of a Plutonium-Bearing Silicate Glass from Rocky Flats Ash Heel and (b) EDS Analysis of Glassy Phase 
TABLE 2.7 Quantitative

Analysis of Plutonium-

Bearing Glassy Phases in

Rocky Flats Ash Heel

\begin{tabular}{ccc}
\hline \multicolumn{3}{c}{ Element, wt\% } \\
\hline $\mathrm{Si}$ & $\mathrm{Al}$ & $\mathrm{Pu}$ \\
\hline 62.8 & 13.9 & 23.3 \\
68.3 & 7.3 & 24.4 \\
68.5 & 7.2 & 24.3 \\
69.1 & 4.1 & 26.7 \\
20.0 & 3.8 & 76.0 \\
\hline
\end{tabular}

of clays and first row transition metals [EDDINGS]. In contrast, efforts to incorporate 7 wt $\%$ plutonium in a Savannah River Laboratory Type 202 glass resulted in phase separation [BATES1995]. The ability of a glass to incorporate plutonium depends critically on the role of other ions. There were some signs of weathering of the glassy plutonium silicate particles found in the residues. Some of the speckled-looking particles in Fig. 2.17 that surround the glassy silicate may be corrosion products. However, no clay material was observed surrounding the glassy phase. Such observations indicate that the plutonium may not be stable enough in the residues for long-term storage. Note also the presence of a plutonium-aluminum particle in Fig. 2.17. Plutonium oxide may be reduced by aluminum to produce a plutonium-aluminum alloy according to the reaction [CLEVELAND-1979]:

$$
3 \mathrm{PuO}_{2}+(4+\mathrm{n}) \mathrm{Al} \rightarrow 3 \mathrm{Pu} \cdot \frac{\mathrm{n}}{3} \mathrm{Al}+2 \mathrm{Al}_{2} \mathrm{O}_{3}
$$

The rate of this reaction is slow, but it could have occurred during the incineration process.

Table 2.5 also shows electron diffraction data from an aluminoplutonium phase which was found in the vicinity of the plutonium glass phase. The EDS from this phase is shown in Fig. 2.18.

\subsubsection{Sand, Slag, and Crucible Samples}

More zirconium oxide particles (also containing cerium) were found in the sand, slag, and crucible samples and the ground LECO crucible sample than in the ash and ash heel samples. This material comes from the zirconia used to crush the samples in an effort to liberate the plutonium, and is therefore an artifact. Figures $2.19 \mathrm{a}$ and $2.19 \mathrm{~b}$ show plutonium particles in the sand, slag, and 


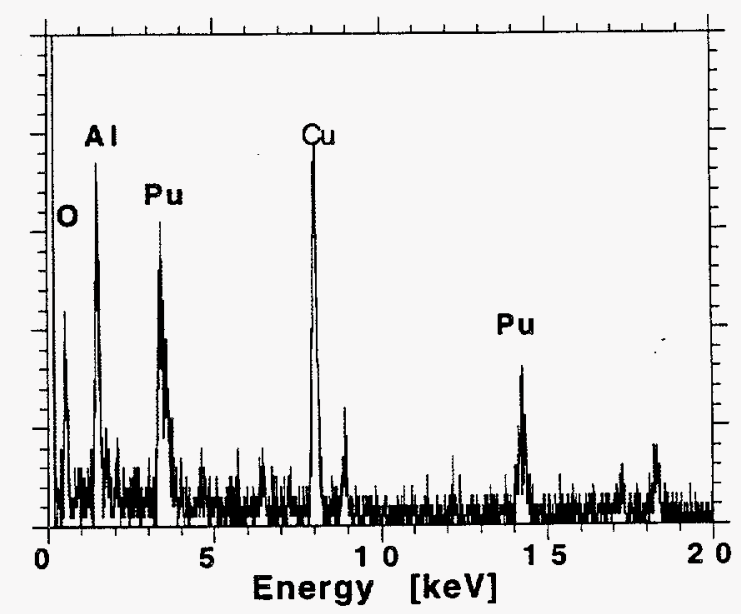

FIGURE 2.18 EDS Analysis of AluminoPlutonium Phase from Rocky Flats

Ash Heel

TABLE 2.8 Electron Diffraction from Plutonium Oxide in LECO Crucible

Literature d-Spacings, $\AA$

\begin{tabular}{llllll}
$\begin{array}{c}\text { Experimental } \\
\mathrm{d}-\text { Spacings, } \AA\end{array}$ & $\begin{array}{c}\text { Error } \\
\pm \AA\end{array}$ & $\mathrm{Pu}_{4} \mathrm{O}_{7}{ }^{\mathrm{a}}$ & $\mathrm{PuO}_{2-\mathrm{x}}{ }^{\mathrm{a}}$ & $\mathrm{PuO}_{2}{ }^{\mathrm{a}}$ & $\begin{array}{c}\mathrm{PuF}_{3} \\
(\text { JCPDS 6-327) }\end{array}$ \\
\hline 5.55 & 0.11 & 5.55 & & & \\
3.54 & 0.06 & 3.89 & & & 3.62 \\
3.16 & 0.05 & 3.18 & 3.14 & 3.1154 & 3.18 \\
1.98 & 0.03 & 1.948 & & & 1.99 \\
1.77 & 0.02 & 1.7877 & & & \\
\hline
\end{tabular}

a From [ROOF]. 

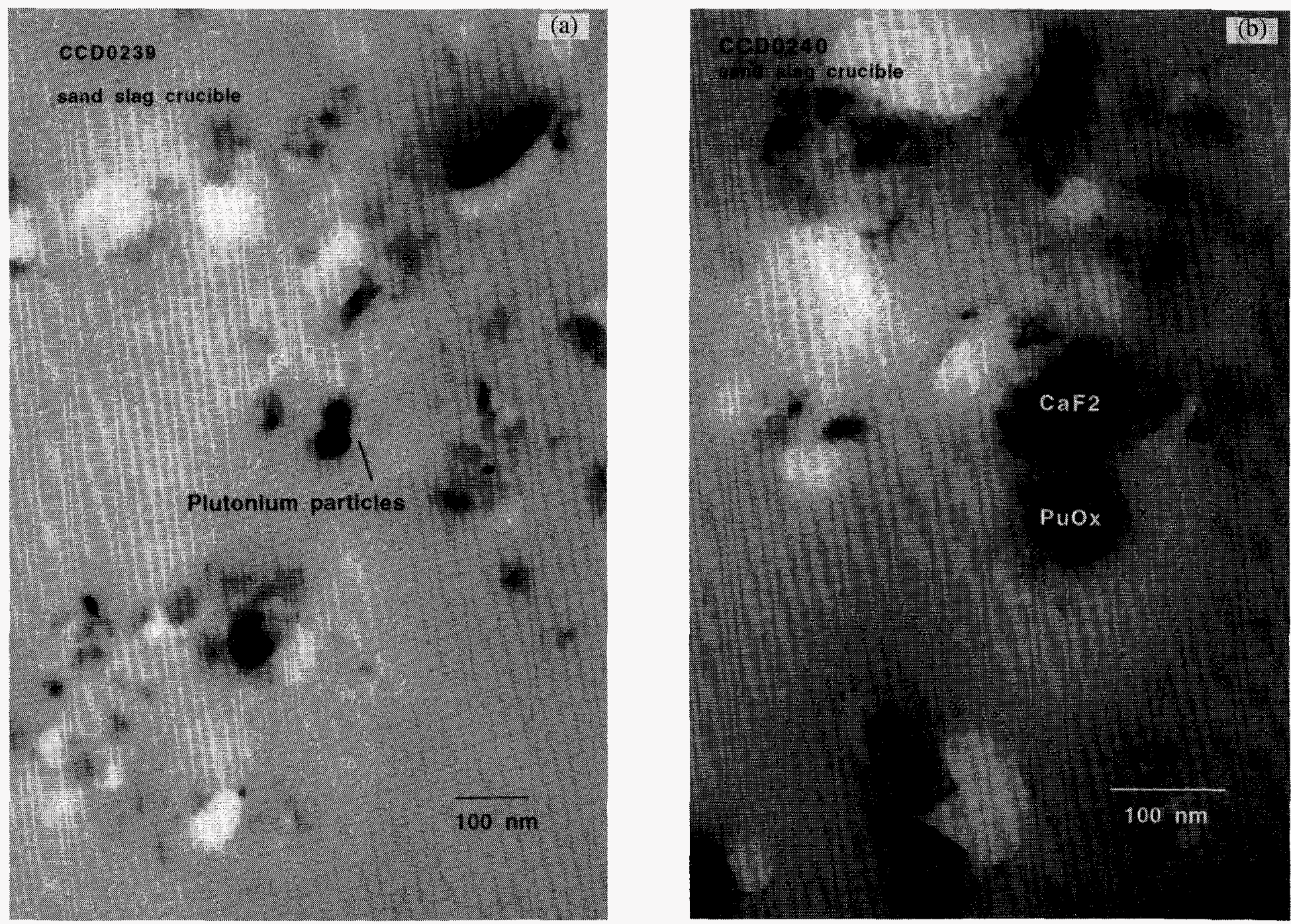

FIGURE 2.19 Analyses of Plutonium Oxide and Other Particles from Sand, Slag, and Crucible Samples. (a-b) TEM images of plutonium oxide and other particles. (Figure and caption continue.) 

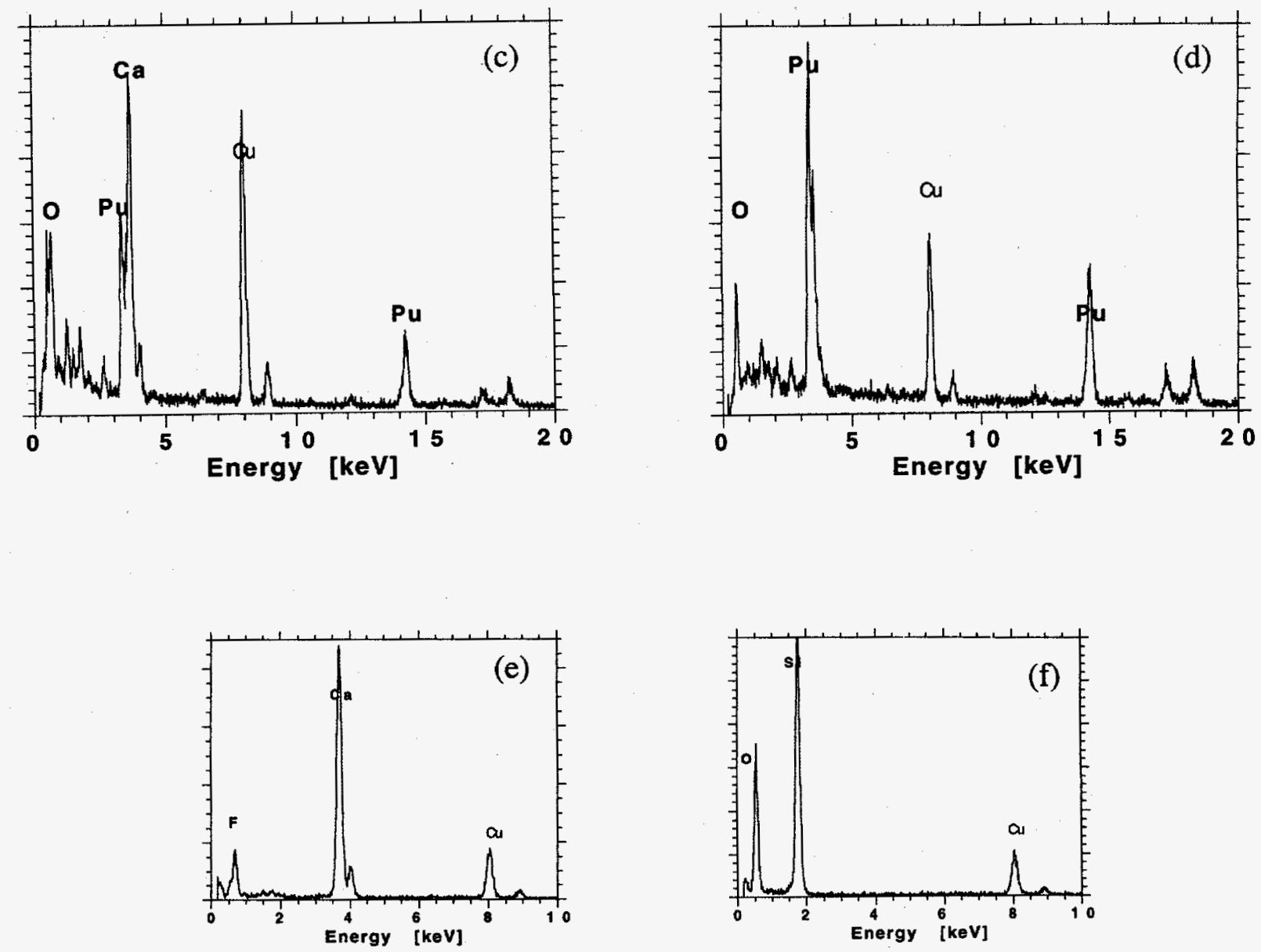

FIGURE 2.19 (Cont.) (c-d) EDS analysis of plutonium phases: (c) $\mathrm{PuO}_{2-\mathrm{x}}$ and fluorite; (d) $\mathrm{PuO}_{2-z^{*}}$ (e-f) EDS analysis of non-plutonium-bearing particles: (e) fluorite; (f) quartz. 


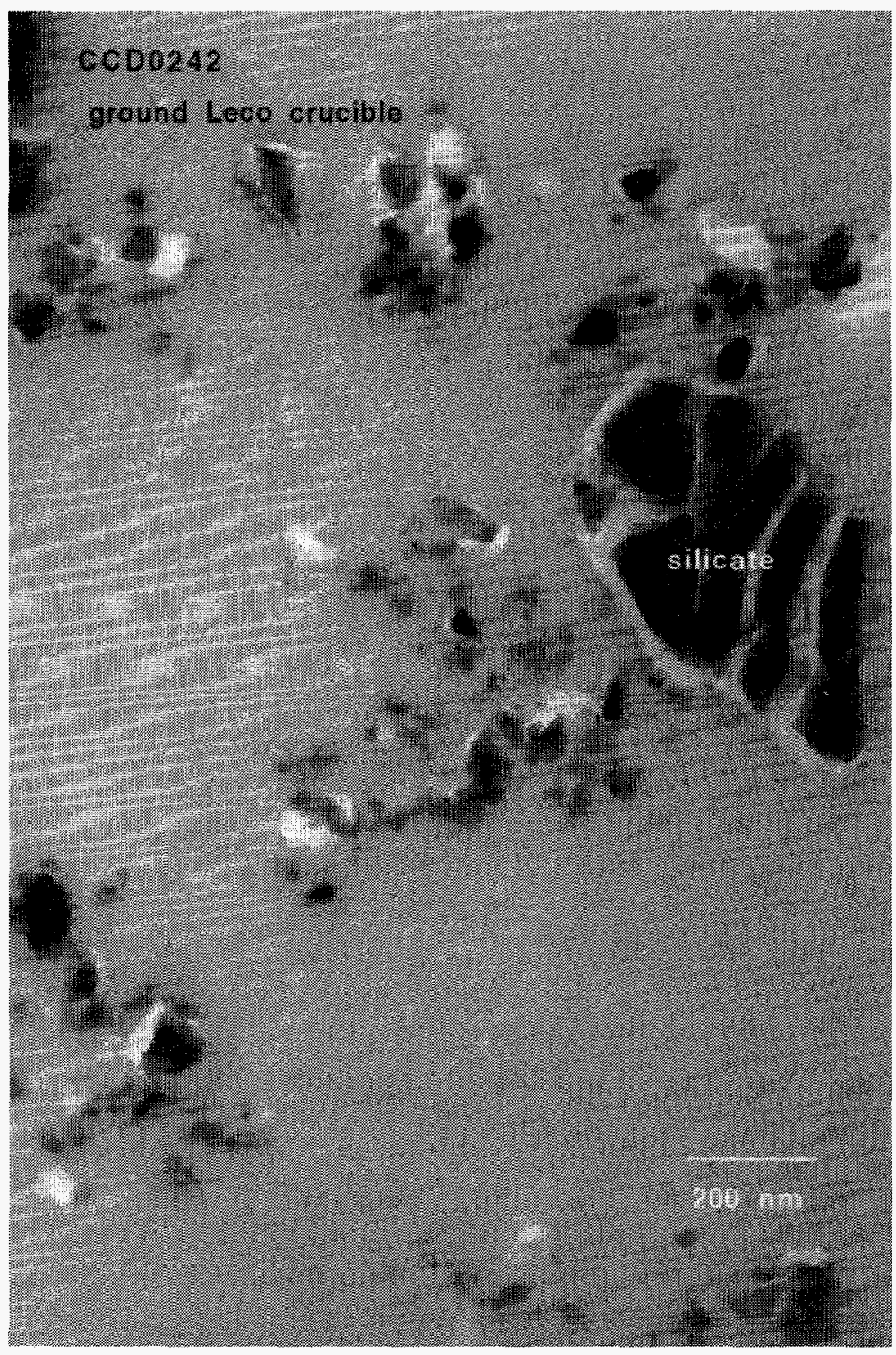

FIGURE 2.20 TEM Images of Plutonium Oxide and Other Particles from Ground LECO Crucible Sample 

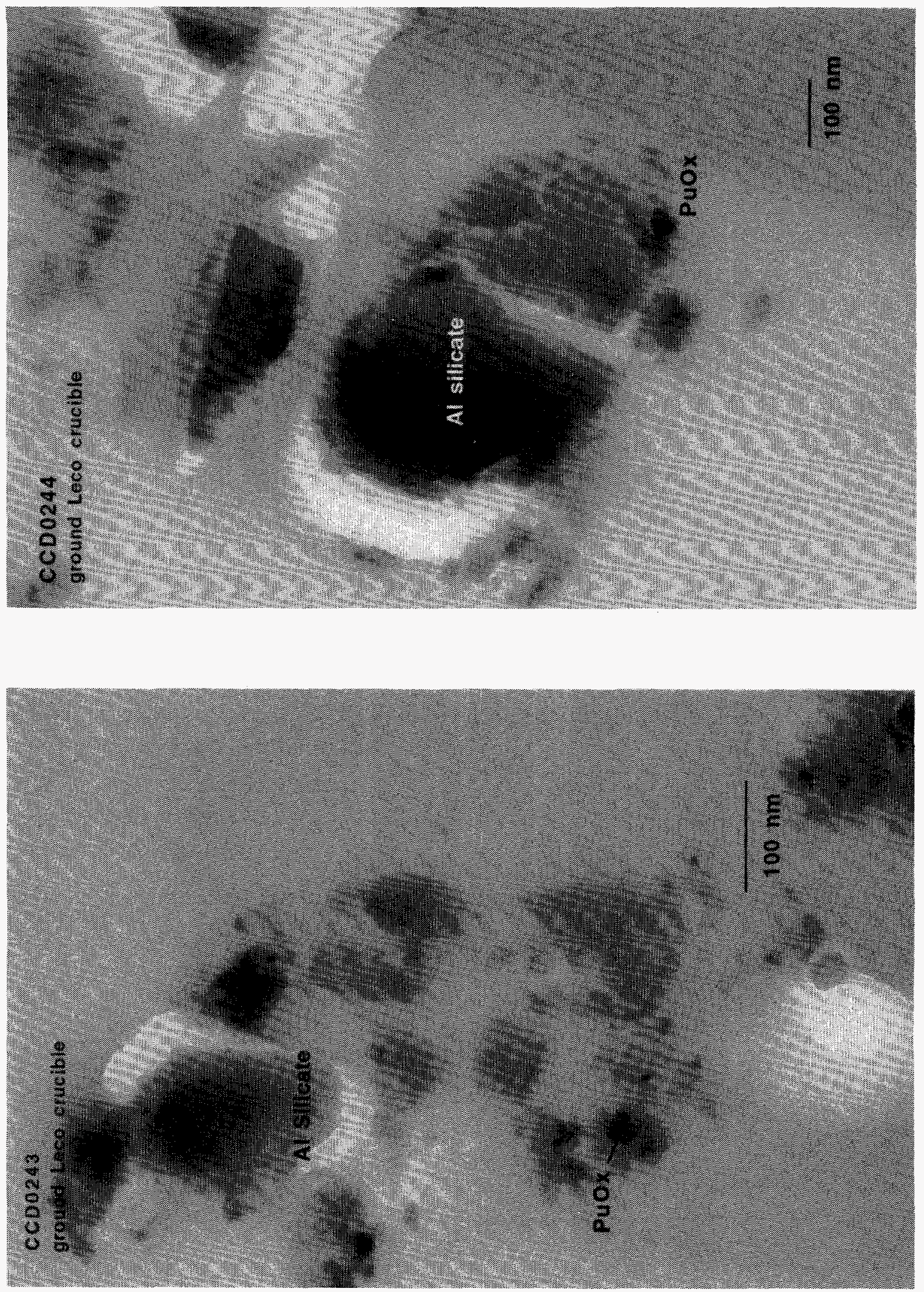

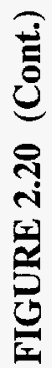



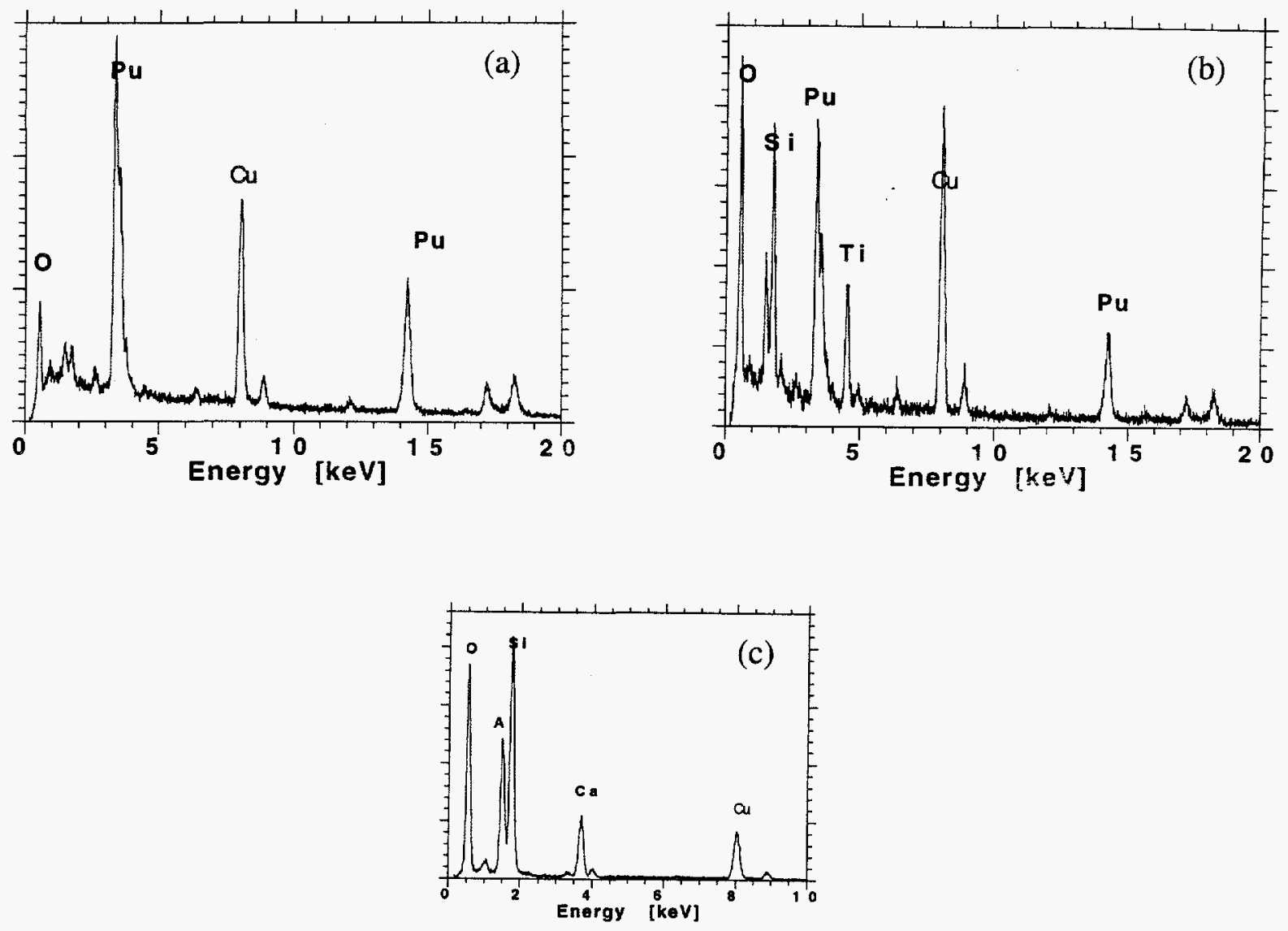

FIGURE 2.21 EDS Analyses of (a) Plutonium Oxide Phase, (b) Plutonium Silicotitanate Phase, and (c) Non-Plutonium-Bearing Particles Containing Calcium Aluminosilicate (possibly anorthite) in Ground LECO Crucible Sample 
crucible samples. In Figs. 2.19c-2.19, an EDS analysis of a plutonium oxide and calcium fluoride (fluorite) particle is shown. The TEM images of these particles show that they were in close proximity. The ultramicrotome causes few changes in the position of particles in the samples, although it can fracture and deform some phases. The plutonium particles in this sample, and also in the ground LECO crucible sample, tended to be smaller than in the Los Alamos samples. Nonplutonium-bearing particles were identified as silica and fluorite.

\subsubsection{Ground LECO Crucible}

The plutonium particles in this sample were very small. (10-20 nm in diameter) and sometimes so closely associated with other phases that it was difficult to determine the composition; however, we believe that most of the plutonium is in the form of a plutonium oxide. Figure 2.20 shows plutonium particles from the ground LECO crucible sample and demonstrates both the small size of these particles and how they have attached themselves to other, larger particles. The EDS analysis of plutonium phases found in the sample is shown in Figs. $2.21 \mathrm{a}$ and $2.21 \mathrm{~b}$. Table 2.8 displays electron diffraction data from the plutonium oxide particles found in the LECO crucible samples. The literature values for a plutonium fluoride are included. The match with the experimental data is fairly good, illustrating the need to have access to both compositional and structural data to make a positive identification of a phase. Calcium aluminosilicates (see Fig. 2.21c) and iron particles were also observed in the residue samples.

\subsection{DISCUSSION}

The most abundant plutonium-bearing phase found in all the waste samples was a reduced plutonium oxide. Plutonium oxide has the fluorite structure (space group $\mathrm{Fm} 3 \mathrm{~m}$ ) and is isomorphous with uraninite, thoria, and ceria. The cubic unit cell $(\mathbf{a}=0.5396 \mathrm{~nm})$ contains four plutonium atoms and eight oxygen atoms. The unit cell parameters are very similar to those of uraninite. Plutonium oxide loses a small amount of oxygen at temperatures of $1100-1200^{\circ} \mathrm{C}$ in a reducing atmosphere. The reduced oxide $\left(\mathrm{PuO}_{2-\mathrm{x}}\right)$ undergoes a eutectoid reaction at $300^{\circ} \mathrm{C}$ that results in the formation of $\mathrm{PuO}_{1.98}$ and $\mathrm{Pu}_{2} \mathrm{O}_{3+y}$, where $\mathrm{x}$ is about 0.3 and $\mathrm{y}$ is greater than 0.24 [CHIKALLA]. The XRD data showed that these are both cubic phases. Roof reported the existence of a cubic $\mathrm{Pu}_{4} \mathrm{O}_{7}$ phase, sometimes also termed $\mathrm{Pu}_{2} \mathrm{O}_{3}$ [ROOF]; however, this phase is not indicated on the $\mathrm{Pu} / \mathrm{O}$ phase diagram and the reflections given in the literature are not distinct.

Although the major phase in all the samples was a reduced plutonium oxide, there was evidence of other plutonium-bearing phases. In the Rocky Flats ash samples, we observed plutonium-bearing aluminosilicates, plutonium silicotitanate phases, an aluminum-plutonium phase, and a glassy aluminosilicate phase containing around $10 \mathrm{wt} \%$ plutonium. The high-silica glassy 
plutonium phase, and other plutonium silicates, would have formed in the incinerator at temperatures of $800-900^{\circ} \mathrm{C}$.

Characterization of the plutonium incinerator wastes proved difficult because the database of plutonium phases, other than simple oxides and halides, is extremely limited. Further analysis will be required to determine the exact form of some of the plutonium-bearing phases found in this study. Even if these phases are identified, however, it will not be possible to predict their long-term stability in the absence of any thermodynamic information.

The plutonium present in ash may be in a relatively stable form, although its size and the nature of the ash material raises some concerns regarding the wastes' ability to retain the plutonium. The plutonium should probably be recovered and vitrified, perhaps by using a minimum additive waste stabilization process, in which other waste materials are added to provide a composition suitable for making a glass. This study has provided a detailed description of the ash residues at Rocky Flats and Los Alamos National Laboratory, information which will be useful in the effort to formulate a durable glass waste form or ceramic waste form for disposal in a geological repository. 


\section{REFERENCES}

AVENS

L. R. Avens, U. F. Gallegos, and J. T. McFarlan, "Magnetic Separation as a Plutonium Residue Enrichment Process," presented at the Sixth Symposium on Separation Science and Technology for Energy Applications, Knoxville, TN, Oct. 22-27, 1989.

BATES-1992

J. K. Bates, J. P. Bradley, A. Teetsov, C. R. Bradley, and M. Buchholtz ten Brink, "Colloid Formation during Waste Glass Reaction: Implications for Nuclear Waste Disposal," Science, 256, 645 (1992).

\section{BATES-1995}

J. K. Bates, J. Emery, J. C. Hoh, and T. R. Johnson, "Performance of High Plutonium Containing Glasses for the Immobilization of Surplus Fissile Materials," International Symposium on the Environmental Issues and Waste Management Technologies in Ceramic and Nuclear Industry, 97th Annual Meeting of the American Ceramic Society, Cincinnati, OH, April 30-May 2 (1995).

\section{BLUM}

T. W. Blum, R. G. Behrens, V. J. Salazar, and P. K. Nystrom, A Small-Scale Study on the Dissolution and Anion-Exchange Recovery of Plutonium from Rocky Flats Plant Incinerator Ash, Los Alamos National Laboratory Report LA-11747 (1989).

\section{BUCK-1994}

E. C. Buck, N. L. Dietz, and N. R. Brown, Analytical Electron Microscopy Study of Ferñald Soils, Argonne National Laboratory Report ANL-94/41 (1994).

\section{BUCK-1995}

E. C. Buck, N. L. Dietz, and J. K. Bates, "Uranium-Contaminated Soils: Ultramicrotomy and Electron Beam Analysis," Microscopy Research and Technique, 31, 174-181 (1995).

\section{CHIKALLA-1964}

T. D. Chikalla, C. E. McNeilly, and R. E. Skavdahl, "The Plutonium-Oxygen System," J. Nuc. Mat., 12, 1331 (1964).

\section{CHRISTENSEN}

D. C. Christensen, D. F. Bowersox, B. J. McKerley, and R. L. Nance, Wastes from Plutonium Conversion and Scrap Recovery Operations, Los Alamos National Laboratory report LA-1 1069-MS (1988). 
CLEVELAND-1979

J. M. Cleveland, The Chemistry of Plutonium, American Nuclear Society, La Grange Park, IL (1979), pp. 291-318.

CLEVELAND-1981

J. M. Cleveland and T. F. Rees, "Characterization of Plutonium in Maxey Flats Radioactive Trench Leachates," Science, 212, 1506-1509 (1981).

\section{CLIFF}

G. Cliff and G. W. Lorimer, "The Quantitative Analysis of Thin-Sections," J. Micro., 103, 203 (1975).

\section{EDDINGS}

E. G. Eddings, J. S. Lighty, and J. A. Kozinski, "Determination of Metal Behavior during the Incineration of a Contaminated Montmorillonite Clay," Environ. Sci. Tech., 28, 1791 (1994).

\section{GRAY}

L. W. Gray and T. Kan, "Immobilization as a Route to Surplus Fissile Materials Disposition," International Symposium on the Environmental Issues and Waste Management Technologies in Ceramic and Nuclear Industry, 97th Annual Meeting of the American Ceramic Society, Cincinnati, $\mathrm{OH}$, April 30-May 2 (1995).

\section{LOBSENZ}

G. Lobsenz, The Energy Daily, 22 [129], 1 (1994).

\section{MANHURON-MANDARD-1994}

X. Machuron-Mandard and C. Madic, "Basic Studies on the Kinetics and Mechanism of the Rapid Reaction of Plutonium Dioxide under Reducing Conditions in Acidic Media," J. Alloys and Compounds, 213/214, 100 (1994).

\section{ROOF}

R. B. Roof, X-Ray Diffraction Data for Plutonium Compounds, Los Alamos National Laboratory report LA-11619 (1989).

\section{SANCHEZ}

A. L. Sanchez, J. W. Murray, and T. H. Sibley, "The Adsorption of Plutonium IV and V on Goethite," Radiochimica Acta, 49, 2297-2307 (1985). 


\section{ZEVENBERGEN}

C. Zevenbergen, J. P. Bradley, T. Vander-Wood, R. S. Brown, L. P. van Reeuwijk, and R. D. Schuiling, "Microanalytical Investigation of Mechanism of Municipal Solid Waste Bottom Ash Weathering," Microbeam Analysis, 3, 125 (1994). 


\section{ACKNOWLEDGMENTS}

Funding for this work was provided by the Division of Advanced Energy Projects, U.S. Department of Energy, Dr. Walter M. Polansky, Director.

The authors would like to thank the following people for providing samples of the plutonium residues used in this study: Dr. Larry Givens of Los Alamos National Laboratory, Dr. Mel Coopes of Lawrence Livermore National Laboratory, Bob Salley of Westinghouse Hanford, and Dr. Dean Pierce of Argonne National Laboratory. 


\section{PART 3: CONCLUSIONS}

Chemical analysis of ash and ash heels from Los Alamos and Rocky Flats show that these materials contain a wide variety of elements. Those components present at $1 \mathrm{wt} \%$ or greater in ash samples were $\mathrm{Al}, \mathrm{C}, \mathrm{Ca}, \mathrm{Cl}, \mathrm{Cr}, \mathrm{Fe}, \mathrm{K}, \mathrm{Mg}, \mathrm{Mn}, \mathrm{Mo}, \mathrm{Na}, \mathrm{Ni}, \mathrm{Pu}, \mathrm{Si}$, and $\mathrm{Zn}$. The only major difference between the Rocky Flats and Los Alamos ashes was that $\mathrm{Ba}, \mathrm{Pb}$, and $\mathrm{Ti}$ were observed in the Rocky Flats ash samples.

Results from sieving experiments indicate that no major fractionation of plutonium occurs during simple size classification. Thus, concentrating plutonium in a small-particle-size fraction of the ash does not appear feasible without prior physical treatment such as grinding.

The combination of chemical analysis and analytical electron microscopy shows that the plutonium phases in ash are refractory and that exposed plutonium phases remain after dissolution with $\mathrm{HNO}_{3} / \mathrm{HF}$. In addition, plutonium phases that were completely surrounded by complex silicate matrices were observed in the ash heels, suggesting that plutonium recoveries could be improved by fine grinding prior to the implementation of chemical recovery techniques.

Even in other types of plutonium-bearing materials, such as LECO crucibles and foundry residues, much of the plutonium appears to be attached to larger phases such as silicates. This phenomenon appears to be common to all samples.

Ultramicrotomy has been found to be effective at sectioning through plutonium oxide and providing large thin sections. The excellent spatial resolution provided by this technique, combined with the ability to extract chemical and structural information, makes AEM a much more suitable tool for examining the residue samples than the traditional methods of XRD. Because the phases are small and surrounded by many other crystalline phases, structural analysis of the plutonium oxide is extremely difficult. In addition, amorphous phases cannot be detected with XRD. The AEM study has showed that the plutonium oxide is mainly in the form of a reduced oxide, possibly $\mathrm{Pu}_{4} \mathrm{O}_{7}$; however, several other types of phases are present, including amorphous phases. This may have implications for removal of plutonium from the ash and immobilization by vitrification. 


\section{Distribution for ANL-95/35}

Internal:
J. M. Andrew
N. L. Dietz
C. C. McPheeters
J. K. Bates
J. E. Harmon
K. M. Myles
J. E. Battles
J. E. Helt
D. L. Bowers
G. K. Johnson
R. D. Pierce
E. C. Buck (10)
T. R. Krause
M. J. Steindler
D. J. Chaiko (10)
J. J. Laidler
J. C. Cunnane
G. Maraman
C. E. Till
G. F. Vandegrift
TIS Files

\section{External:}

DOE-OSTI (2)

ANL-E Library (2)

ANL-W Library

Manager, Chicago Operations Office, DOE

A. Bindokas, DOE-CH

J. Haugen, DOE-CH

Chemical Technology Division Review Committee Members:

E. R. Beaver, Monsanto Company, St. Louis, MO

D. L. Douglas, Consultant, Bloomington, MN

R. K. Genung, Oak Ridge National Laboratory, Oak Ridge, TN

J. G. Kay, Drexel University, Philadelphia, PA

G. R. St. Pierre, Ohio State University, Columbus, $\mathrm{OH}$

J. Stringer, Electric Power Research Institute, Palo Alto, CA

J. B. Wagner, Arizona State University, Tempe, AZ

M. G. Adamson, Lawrence Livermore National Laboratory, Livermore, CA

J. Apple, Westinghouse Hanford Company, Richland, WA

J. A. Apps, Lawrence Berkeley Laboratory, Berkeley, CA

L. R. Avens, Los Alamos National Laboratory, Los Alamos, NM

G. S. Barney, Westinghouse Hanford Operations, Richland, WA

C. A. Barrington, Westinghouse Hanford Company, Richland, WA

R. G. Behrens, Los Alamos National Laboratory, Los Alamos, NM

J. D. Berger, Westinghouse Hanford Company, Richland, WA

E. T. Bramlitt, Defense Nuclear Agency, Kirtland AFB, NM

J. L. Burnett, USDOE, Office of Basic Energy Sciences, Germantown, MD

N. Castaneda, USDOE, Rocky Flats Field Office, Golden, CO

D. C. Christensen, Los Alamos National Laboratory, Los Alamos, NM

R. H. Condit, Lawrence Livermore National Laboratory, Livermore, CA

T. D. Cooper, Westinghouse Hanford Company, Richland, WA

M. S. Coops, Lawrence Livermore National Laboratory, Livermore, CA

J. L. Cox, Pacific Northwest Laboratory, Richland, WA

W. R. Dworzak, Los Alamos National Laboratory, Los Alamos, NM 
M. Eagle, U.S. Environmental Protection Agency, Washington, DC

C. W. Francis, Oak Ridge National Laboratory, Oak Ridge, TN

T. Fryberger, USDOE, Office of Technology Development, Germantown, MD

J. S. Garfield, Westinghouse Hanford Company, Richland, WA

M. A. Gerber, Pacific Northwest Laboratory, Richland, WA

D. Gombert, Lockheed Idaho Technology Company, Idaho Falls, ID

W. Gray, Pacific Northwest Laboratory, Richland, WA

J. Hart, USDOE, FUSRAP, Oak Ridge, TN

J. Hyde, USDOE, Office of Technology Development, Washington, DC

D. G. Karraker, Westinghouse Savannah River Company, Aiken, SC

D. L. Keairns, Westinghouse Science and Technology Center, Pittsburgh, PA

C. M. Kronvall, Westinghouse Hanford Operations, Richland, WA

W. L. Kuhn, Pacific Northwest Laboratory, Richland, WA

D. E. Kurath, Pacific Northwest Laboratory, Richland, WA

G. J. Lumetta, Pacific Northwest Laboratory, Richland, WA

J. S. McCormack, Westinghouse Hanford Company, Richland, WA

C. P. McGinnis, Oak Ridge National Laboratory, Oak Ridge, TN

D. Morris, Los Alamos National Laboratory, Los Alamos, NM

A. C. Muscatello, LATO Office, Rocky Flats Plant, Golden, CO

R. M. Orme, Westinghouse Hanford Company, Richland, WA

M. Palmer, Los Alamos National Laboratory, Los Alamos, NM

W. M. Polansky, USDOE, Division of Advanced Energy Projects, Germantown, MD

C. Purdy, USDOE, Office of Technology Development, Germantown, MD

B. Salley, Westinghouse Hanford Company, Richland, WA

M. J. Schliebe, Westinghouse Hanford Operations, Richland, WA

V. Tidwell, USDOE, Sandia National Laboratories, Albuquerque, NM

E. Van Deventer, Shorewood, IL

R. E. Vandercook, Westinghouse Hanford Company, Richland, WA

S. Warren, USDOE, Office of Environmental Restoration, Germantown, MD

J. Watson, Oak Ridge National Laboratory, Oak Ridge, TN 